\title{
Metabolism of 6:2 Fluorotelomer Alcohol by CYP 2A6
}

By

\author{
Oluwadamilola Daramola \\ A thesis submitted to the Department of Chemistry \\ in partial fulfilment of the requirements for the degree of \\ Master of Science
}

In

Chemistry (Chemical and Environmental Toxicology)

Carleton University

Department of Chemistry

Ottawa, Canada

(C) Copyright 2021, Oluwadamilola Daramola 


\section{Abstract}

For well-known toxicants such as perfluorooctanoic acid (PFOA) and its precursor, 8:2 fluorotelomer alcohol (8:2 FTOH), identification of the CYPs driving the metabolism, from the FTOH to the PFOA, could be used to identify the possibility of localized toxicity. This is based on the varying distribution of CYPs in organs and cell membranes. Due to environmental regulations, PFOA and 8:2 FTOH have been phased out in favour of compounds with shorter fluorinated chain lengths, considered less bioaccumulative and toxic. However, there is currently insufficient knowledge of enzyme catalysed metabolism of these replacements. One replacement for 8:2 FTOH is the 6:2 $\mathrm{FTOH}^{[4]}$. This project identifies CYP 3A4 and CYP 2A6 as possible cytochrome P450s responsible for the phase I metabolism of 6:2 FTOH. A Michaelis Menten curve is generated for the metabolism of 6:2 FTOH by CYP 2A6 metabolism. This provided a $K_{\mathrm{M}}$ and $\mathrm{V}_{\mathrm{Max}}$ of $4076.4 \pm 581.9 \mathrm{ng} / \mathrm{mL}$ and $68.8 \pm 2.8 \mathrm{ng} / \mathrm{mL} / \mathrm{min}$, respectively. Once inhibited with $35 \mu \mathrm{M}$ of tranylcypromine, $\mathrm{HCl}$, a selective inhibitor of CYP 2A6, the $K_{\mathrm{M}}$ and $\mathrm{V}_{\mathrm{Max}}$ were determined to be $8796.2 \pm 1366.1 \mathrm{ng} / \mathrm{mL}$ and $69.5 \pm 4.1 \mathrm{ng} / \mathrm{mL} / \mathrm{min}$, representing competitive inhibition. We further demonstrated that CYP 2A6 was responsible for 6:2 FTOH metabolism using human recombinant assays with purified CYP 2A6. These assays yielded a 6:2 FTOH metabolic conversion rate of $0.42 \mathrm{ng} / \mathrm{mL} / \mathrm{min}$. This rate significantly decreased with the addition of Tranylcypromine $\mathrm{HCl}$. This confirms CYP 2A6 as an active enzyme for the metabolism of 6:2 FTOH in the human liver. 


\section{Acknowledgement}

Firstly, I would like to thank my family Yetunde, Michael, DJ and Morinsola Daramola. They stood by and supported me throughout the entirety of my stay in Ottawa. As a newcomer to the city and the school, their constant reassurance during days of doubt and loneliness was invaluable. Ever since I discovered my interest in Chemistry in the $11^{\text {th }}$ grade, they have been an unmovable pillar of support in my life. Whenever I felt lost or unsure about my choice to come to Ottawa my mother will always be there to reassure me. My father would keep my spirits high during the weekend as we discussed the latest results of the Premier League weekend. My Sister and Brother were sources of smiles in time of need.

I would like to also thank my Ottawa based family, the Ayenis. They welcomed me into their lives and treated me as one of their own. In times of holidays or just boring weekends, their home was my home.

I would like to thank my fellow M.Sc. students and friends who made coming into school every day a treat and a delight. Sampson, you were always there when I had problems with incubation or GC analysis. And to the duo Colleens, hanging out in the lab and just chatting the day away would be a memory I will always treasure. And Keegan... you know what you did.

Finally, I would like to thank my supervisor, Amy Rand. I appreciate the chance you gave me to study and learn a lot about environmental toxicity as it was not a focus that I considered. The two years I spent here have been invaluable. 


\section{TABLE OF CONTENTS}

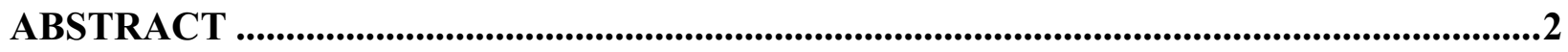

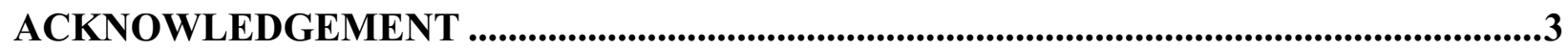

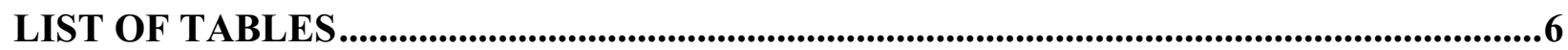

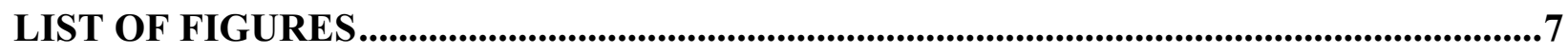

LIST OF SUPPLEMENTARY INFORMATION DATA .....................................................9

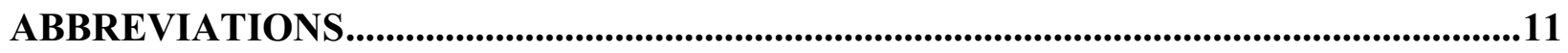

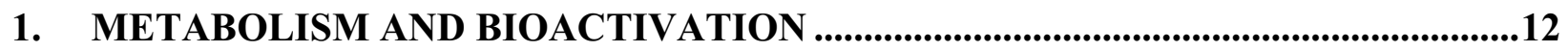

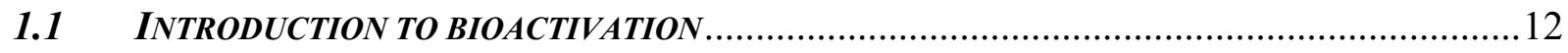

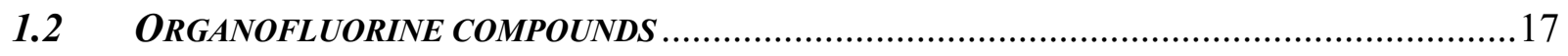

1.3 FLUOROTELOMER ALCOHOLS AND THEIR TRANSFORMATION PRODUCTS .....................21

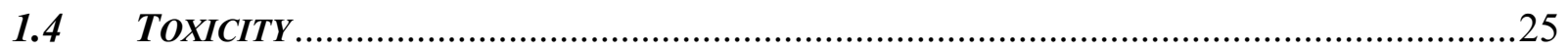

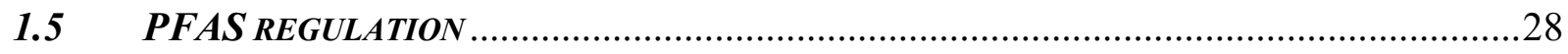

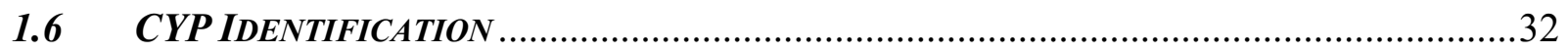

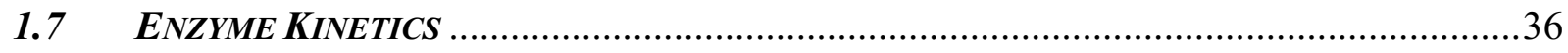

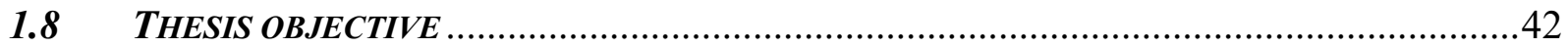

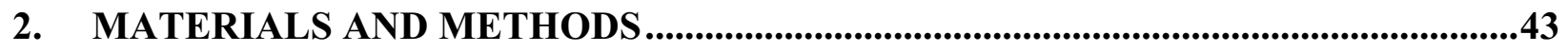

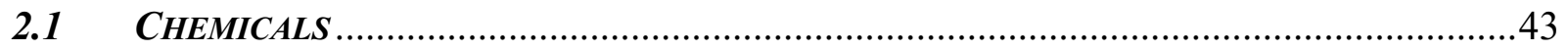

2.2 INCUBATION OF 6:2 FTOH WITH HUMAN LIVER MICROSOMES.................................4

2.3 INCUBATION OF 6:2 FTOH WITH RECOMBINANT HUMAN CYP 2A6........................46

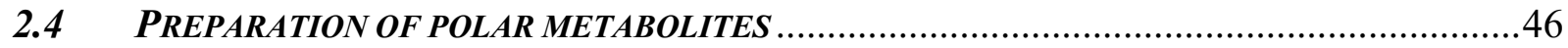




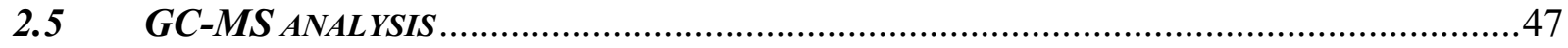

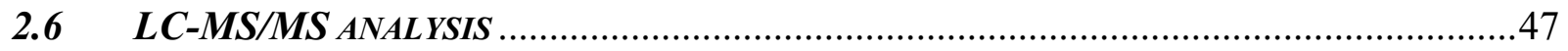

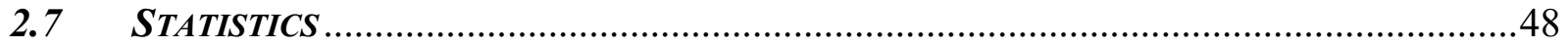

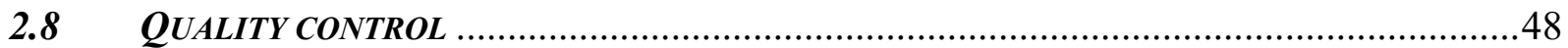

3. 6:2 FTOH METABOLIZES BY CYP 3A4 AND 2A6 IN HUMAN LIVER

MICROSOMES .......................................................................................................................................50

4. UNINHIBITED AND INHIBITED MICHAELIS MENTEN CURVES .......................56

5. 6:2 FTOH METABOLISM IN HUMAN RECOMBINANT CYP 2A6..........................60

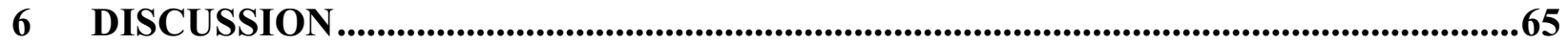

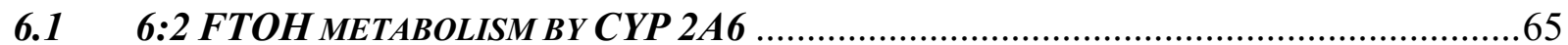

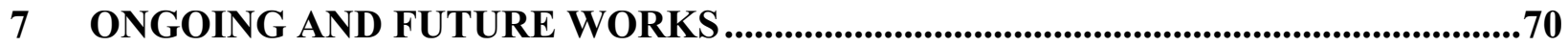

REFERENCES

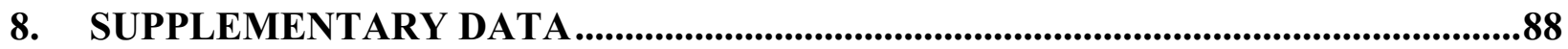




\section{List of Tables}

Table 2.1: The names of the competitive inhibitors and the final concentration observed in the incubation well.

Table 2.2: The limit of detection and limit of quantitation for the 6:2 FTOH and 6:2 FTUCA.

Table 3.1: The p-values corresponding with a one-way ANOVA test between the uninhibited and inhibited 6:2 FTOH metabolic rates at 1,000 and 10,000 ng/mL. "All trials" signify the ANOVA test comparing the uninhibited with all the inhibited trials 54

Table 4.1: $\mathrm{V}_{\mathrm{Max}}$ and $\mathrm{K}_{\mathrm{M}}$ and their respective 95\% confidence interval for both the uninhibited and inhibited Michaelis Menten curve. 58 


\section{List of Figures}

Figure 1.1: The creation of an oxyferryl intermediate within the CYPs heme centre ${ }^{[12]} . . . .14$

Figure 1.2 Conversion of NAD+ to NADPH in an NADPH regeneration system............... 15

Figure 1.4: Distribution of CYPs in the human liver (a) and small intestine (b) ${ }^{[24]} \ldots \ldots \ldots \ldots 17$

Figure 1.5: Possible biotransformation pathway for $6: 2$ FTOH ....................................... 20

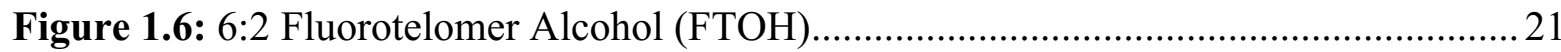

Figure 1.8: Primary route of exposure (ingestion, inhalation and dermal) for 6:2 FTOH and their transportation in the human body. 34

Figure 1.9: Procedure for the preparation of liver microsomes ......................................... 35

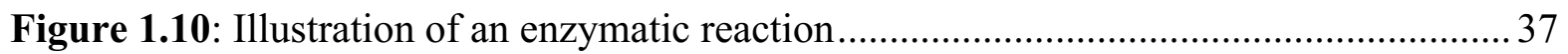

Figure 1.11: An illustration of a Michaelis Menten curve with the constants $K_{\mathrm{M}}$ and $\mathrm{v}_{\max . . .38} 38$

Figure 1.12: The effects of inhibition on the Michaelis Menten curve and the constants $K_{M}$ and $V_{\max }$ 41

Figure 3.1: Metabolic rates of 6:2 FTOH conversion in the presence of competitive inhibitors. The starting concentration of 6:2 FTOH is $1000 \mathrm{ng} / \mathrm{mL}$. Standard deviations were taken from $\mathrm{n}=3$ trials. “*” symbolizes a $\mathrm{p}$-value $<0.05$ in a one-way t-test......................................5. 52

Figure 3.2: Metabolic rates of 6:2 FTOH conversion in the presence of competitive inhibitors. The starting concentration of 6:2 FTOH is $10000 \mathrm{ng} / \mathrm{mL}$. Standard deviations were derived from $\mathrm{n}=3$ trials. "*” symbolizes a $\mathrm{p}$-value $<0.05$ in a one-way t-test 53

Figure 4.1: Michaelis Menten plot of 6:2 FTOH biotransformation in human liver microsomes $(0.20 \mathrm{mg}) .6: 2 \mathrm{FTOH}$ is incubated in the presence of an NADPH regeneration system at $37{ }^{0} \mathrm{C}$ for time points ranging from 0 to 22 hours. The Michaelis Menten constant for the biotransformation in the absence of CYP 2A6 inhibition (blue circles) is $4076.4 \pm 581.9 \mathrm{ng} / \mathrm{mL}$, 
and the maximum rate of $68.8 \pm 2.8 \mathrm{ng} / \mathrm{mL} / \mathrm{min}$. The Michaelis Menten constant in the presence of CYP 2A6 inhibitor (red triangles) is $8796.2 \pm 1366.1 \mathrm{ng} / \mathrm{mL}$, and the maximum rate of $69.5 \pm$ $4.1 \mathrm{ng} / \mathrm{mL} / \mathrm{min}$. The constants were obtained through nonlinear regression using MatLab. Error bars indicate standard deviation acquired from $n=3$ trials

Figure 5.1: Metabolic rates of inhibited and uninhibited 6:2 FTOH conversion in the presence of human recombinant CYP 2A6. The starting concentration of 6:2 FTOH is $1000 \mathrm{ng} / \mathrm{mL}$. The concentration of CYP 2 A6 is $0.3 \mathrm{ng} / \mathrm{mL}$. Standard deviations were derived from $\mathrm{n}=3$ trials. “*” symbolizes a $p$-value $<0.05$ in a one-way t-test when compared to the control. 62

Figure 5.2: Metabolic rates of inhibited and uninhibited 6:2 FTOH conversion in the presence of Human recombinant CYP 2A6. The starting concentration of 6:2 FTOH is $10000 \mathrm{ng} / \mathrm{mL}$. The starting concentration of CYP 2A6 is $0.3 \mathrm{ng} / \mathrm{mL}$. Standard deviations were derived from $\mathrm{n}=3$ trials. "*”" symbolizes a p-value $<0.05$ in a one-way t-test when compared to the control.... 63 Figure 5.3: Metabolic rates of inhibited and uninhibited 6:2 FTOH conversion in the presence of human recombinant CYP 2A6. The starting concentration of $6: 2 \mathrm{FTOH}$ is $10000 \mathrm{ng} / \mathrm{mL}$. The concentration of CYP 2A6 is $0.3 \mathrm{ng} / \mathrm{mL}$. Standard deviations were derived from $\mathrm{n}=3$ trials. 64 Figure 6.1: CYP 2A6 substrates: Nicotine, Coumarin and Cotinine ...................................6 66

Figure 6.2: CYP 3A4 substrates: Acetaminophen and Codeine ........................................ 68 


\section{List of Supplementary Information Data}

Table S1: Spike and recovery of 6:2 FTOH. Spike and recovery are obtained from 6:2 FTOH incubations throughout $2 \mathrm{hrs}$. 88

Table S2: Metabolic rates of 6:2 FTOH conversion in the presence of competitive inhibitors. The starting concentration of $6: 2 \mathrm{FTOH}$ is $1000 \mathrm{ng} / \mathrm{mL}$. Standard deviations are taken from $\mathrm{n}=3$ trials. 89

Table S3: Metabolic rates of 6:2 FTOH conversion in the presence of competitive inhibitors. The starting concentration of $6: 2 \mathrm{FTOH}$ is $10,000 \mathrm{ng} / \mathrm{mL}$. Standard deviations are taken from $\mathrm{n}=3$ trials. 90

Table S4: Metabolic rates of inhibited and uninhibited 6:2 FTOH conversion in the presence of Human recombinant CYP 2A6. The starting concentration of $6: 2 \mathrm{FTOH}$ is $10,000 \mathrm{ng} / \mathrm{mL}$. The starting concentration of CYP $2 \mathrm{~A} 6$ is $0.3 \mathrm{ng} / \mathrm{mL}$. Standard deviations are acquired from $\mathrm{n}=3$ trials. 91

Table S5: Metabolic rates of inhibited and uninhibited 6:2 FTOH conversion in the presence of Human recombinant CYP 2A6. The starting concentration of $6: 2 \mathrm{FTOH}$ is $1000 \mathrm{ng} / \mathrm{mL}$. The starting concentration of CYP $2 \mathrm{~A} 6$ is $0.3 \mathrm{ng} / \mathrm{mL}$. Standard deviations are acquired from $\mathrm{n}=3$

trials. 92

Table S6: Rate and specific rate of 6:2 FTOH in uninhibited and inhibited (CYP 2A6)

microsomes. The inhibition is from the addition of 50.0 $\mu \mathrm{M}$ of Tranylcypromine, HCL. Standard deviations are acquired from $n=3$ trials. 94

Table S7: Metabolic rates of 6:2 FTUCA conversion in the presence of competitive inhibitors. The starting concentration of $6: 2 \mathrm{FTOH}$ is $1000 \mathrm{ng} / \mathrm{mL}$. Standard deviations are taken from $\mathrm{n}=3$ trials. 
Table S8: Metabolic rates of 6:2 FTUCA conversion in the presence of competitive inhibitors. The starting concentration of $6: 2 \mathrm{FTOH}$ is $10,000 \mathrm{ng} / \mathrm{mL}$. Standard deviations are taken from $\mathrm{n}=3$ trials. 95

Table S9: The p-values corresponding with a one-way ANOVA test between the uninhibited and inhibited 6:2 FTUCA production rates at 1,000 and 10,000 ng/mL. "All trials" signify the ANOVA test comparing the uninhibited with all the inhibited trials. 96 
Abbreviations

2-(Perfluoro-7-methyl octyl) ethanol (iso-9:2 FTOH)

Cytochrome P450 (CYP)

Fluorotelomer alcohol (FTOH)

Fluorotelomer aldehyde (FTAL)

Fluorotelomer carboxylic acid (FTCA)

Fluorotelomer unsaturated aldehyde (FTUAL)

Fluorotelomer unsaturated carboxylic acid (FTUCA)

Gas Chromatography (GC)

Internal standard (IS)

Liquid Chromatography (LC)

Maximum Velocity $\left(\mathrm{V}_{\mathrm{Max}}\right)$

Mass spectrometry (MS)

Michaelis-Menten constant $\left(\mathrm{K}_{\mathrm{M}}\right)$

Poly- and Per-fluoroalkyl substances (PFAS)

Perfluoro carboxylic acid (PFCA)

Perfluoroheptanoic acid (PFHpA)

Perfluorohexanoic acid (PFHxA)

Perfluorononanoic acid (PFNA)

Perfluorosulfonate (PFSA)

Perfluorooctanesulfonic acid (PFOS)

Perfluorooctanoic acid (PFOA)

Select ion monitoring (SIM) 


\section{Metabolism and Bioactivation}

\subsection{Introduction to bioactivation}

Metabolism plays a major part in the bioactivation of several commercially available drugs. Prodrugs are sold in an inactive form, and with the help of enzymatic reactions, they are activated. Once activated, they can serve their intended purpose ${ }^{[1]}$. This helps with the targeting of specific components on the cell membrane or specific organs in the human body based on the activating enzyme. Precursors of toxicants follow similar paths to prodrugs. Due to the multitudes of commercially available products, there are a variety of metabolic pathways from a plethora of precursors that could terminate at a toxic metabolite. This increases the difficulty in the environmental regulation of compounds.

Metabolic reactions are grouped into phase I and phase II metabolism ${ }^{[2][3]}$. Phase I involves the addition of a polar functional group or modification of a functional group to yield a more polar compound ${ }^{[2]}$. The addition of a hydroxyl group, an oxygen atom, or the conversion of an alcohol group to a carbonyl centre, are examples of phase I metabolism ${ }^{[2]}$. Phase I metabolism is the first step in the bioactivation of prodrugs ${ }^{[2]}$. It is also the first step in the elimination of lipophilic toxicant ${ }^{[3]}$. As the oxidation increases, the lipophobicity increases and this generally drives the toxicant's elimination from the body. Phase II metabolism, also known as conjugation, is the addition of a functional group or compound to the drug in question ${ }^{[5]}$. Glucuronic acid (Tetrahydroxyoxane-2-carboxylic acid) and glutathione ((2S)-2-Amino-5(\{(2R)-1-[(carboxymethyl)amino]-1-oxo-3-sulfanylpropan-2-yl $\}$ amino)-5-oxopentanoic acid) are examples of compounds used in the conjugation process ${ }^{[5][6]}$. These reactions are primarily nucleophilic and electrophilic substitutions, with the help of phase II enzymes like glucuronosyltransferase (UGT), which is used in turn with glucuronic acid, and glutathione S- 
transferase (GST), which is used in turn with glutathione ${ }^{[7]}$. Carbonyl, hydroxyl, and amines are functional groups highly susceptible to Phase II conjugation reactions ${ }^{[5][6]}$. Phase II metabolism generally results in more efficient depuration of compounds from the exposed organism. The increase in molar mass and polarity after conjugation leads to increased lipophobicity and increased elimination ${ }^{[6]}$.

A common phase I metabolism for drug activation is oxidation catalyzed by cytochrome P450 monooxygenase (CYP) ${ }^{[3]}$. CYPs exist in all kingdoms of life ${ }^{[5]}$. CYPs involved in phase I metabolism are heme-containing enzymes that primarily serve as monooxygenases, where one oxygen atom is transferred to the substrate to yield the oxidized product ${ }^{[8][9]}$. The heme molecule is attached to the centre of the protein via a cysteine thiolate ligand ${ }^{[9]}$. The CYP metabolism involves the creation of an oxyferryl intermediate within the enzyme active site ${ }^{[9]}$. These reactions are aerobic reactions, requiring oxygen as a co-factor. In the presence of diatomic oxygen, they are fuelled by the transfer of two electrons from the molecule nicotinamide adenine dinucleotide phosphate (NADPH) to the CYP, which forms the oxyferryl intermediate, as seen in figure $1.1^{[9]}$. 

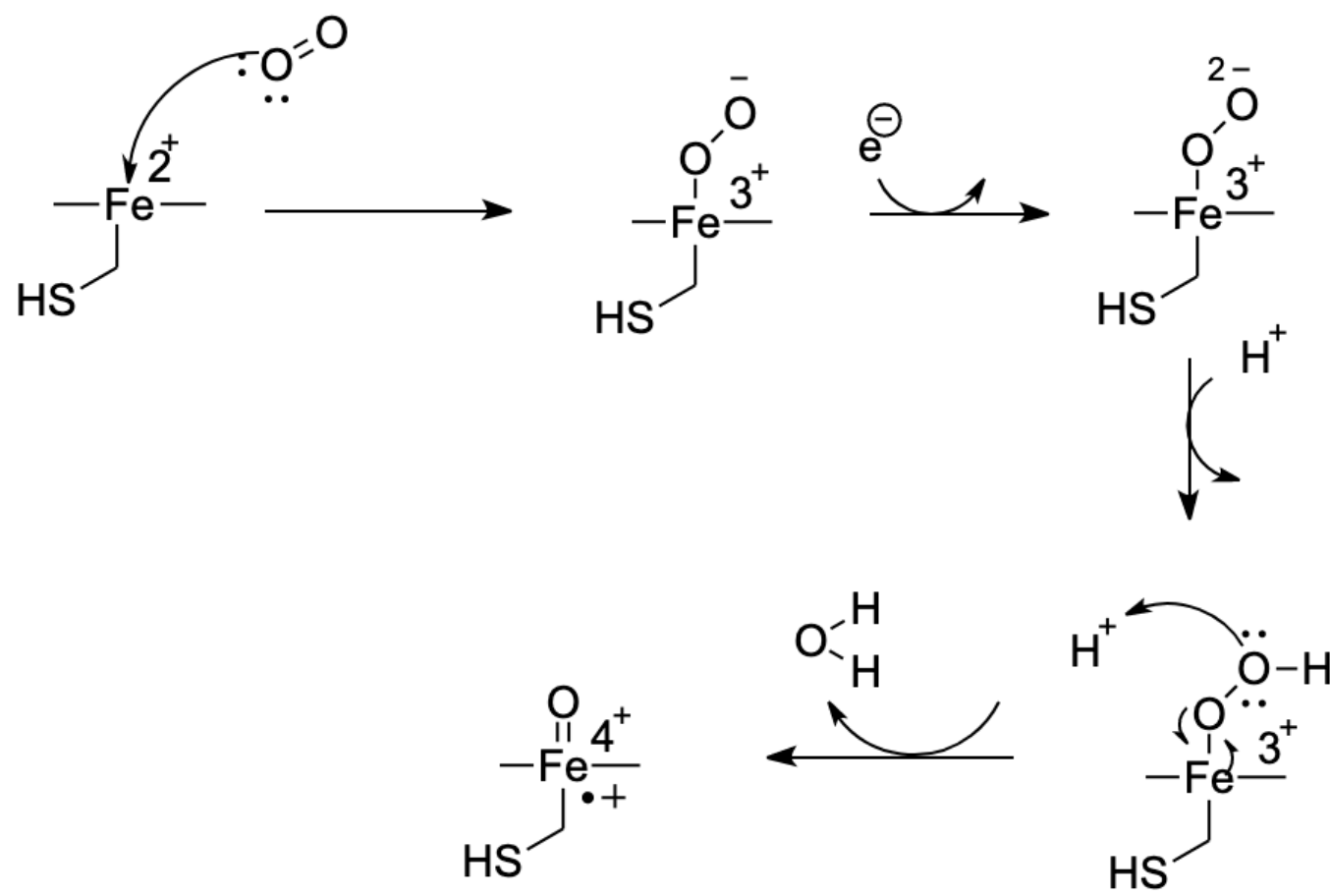

Figure 1.1: The creation of an oxyferryl intermediate within the CYPs heme centre ${ }^{[10]}$.

In in vitro assays that involve phase I oxidative metabolism, a constant concentration of NADPH must be achieved to maintain the reaction ${ }^{[11]}$. Once electrons have been transferred, NADPH is converted to NADP+, as seen in Figure 1.2. 


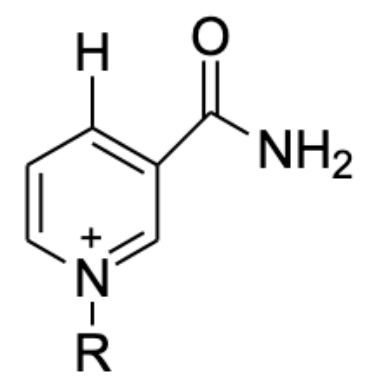

NADP+

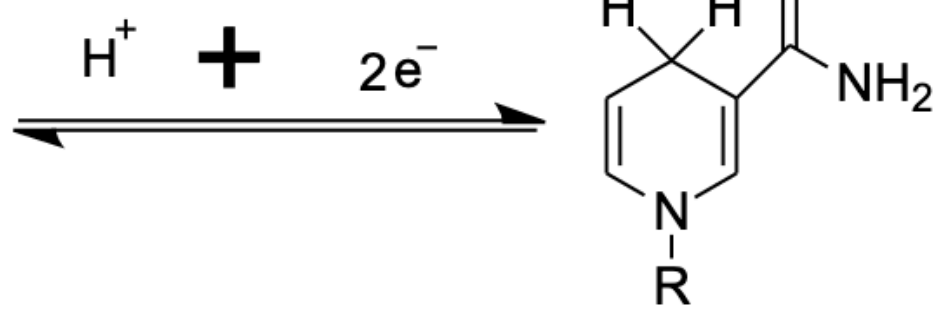

NADPH

Figure 1.2 Conversion of NAD+ to NADPH in an NADPH regeneration system.

The NADP + can be converted back to NADPH through a reduction reaction. The reduction reaction is achieved in what is known as an NADPH regeneration system ${ }^{[12]}$. This system consists of NADP+, glucose-6-phosphate (G6P) as the source of the electron, and glucose-6-phosphate dehydrogenase (d-G6P) to help facilitate the transfer of the electron from G6P to NAD ${ }^{[12]}$. In the presence of an NADPH regeneration system, a phase I metabolism limiting reactant will be the quantity of the substrate.

Two common phase I metabolic reactions are the addition of a hydroxyl group to a sigma $\mathrm{C}-\mathrm{H}$ bond and the oxidation of a polar group. The addition of a hydroxyl group can be performed with the direct addition of the -OH group to the carbon centre. It can also be performed by the creation of an epoxide. The formation of an epoxide is characteristic of the CYP mediated oxidation of an aromatic ring. This increases the polarity of the compound. It can be eliminated through conjugation via phase II metabolic reactions, or in the case of a few compounds, the increase the possibility of further biotic and/or abiotic reactions to yield active products. An example of CYP mediated oxidation is shown in figure 1.3. 

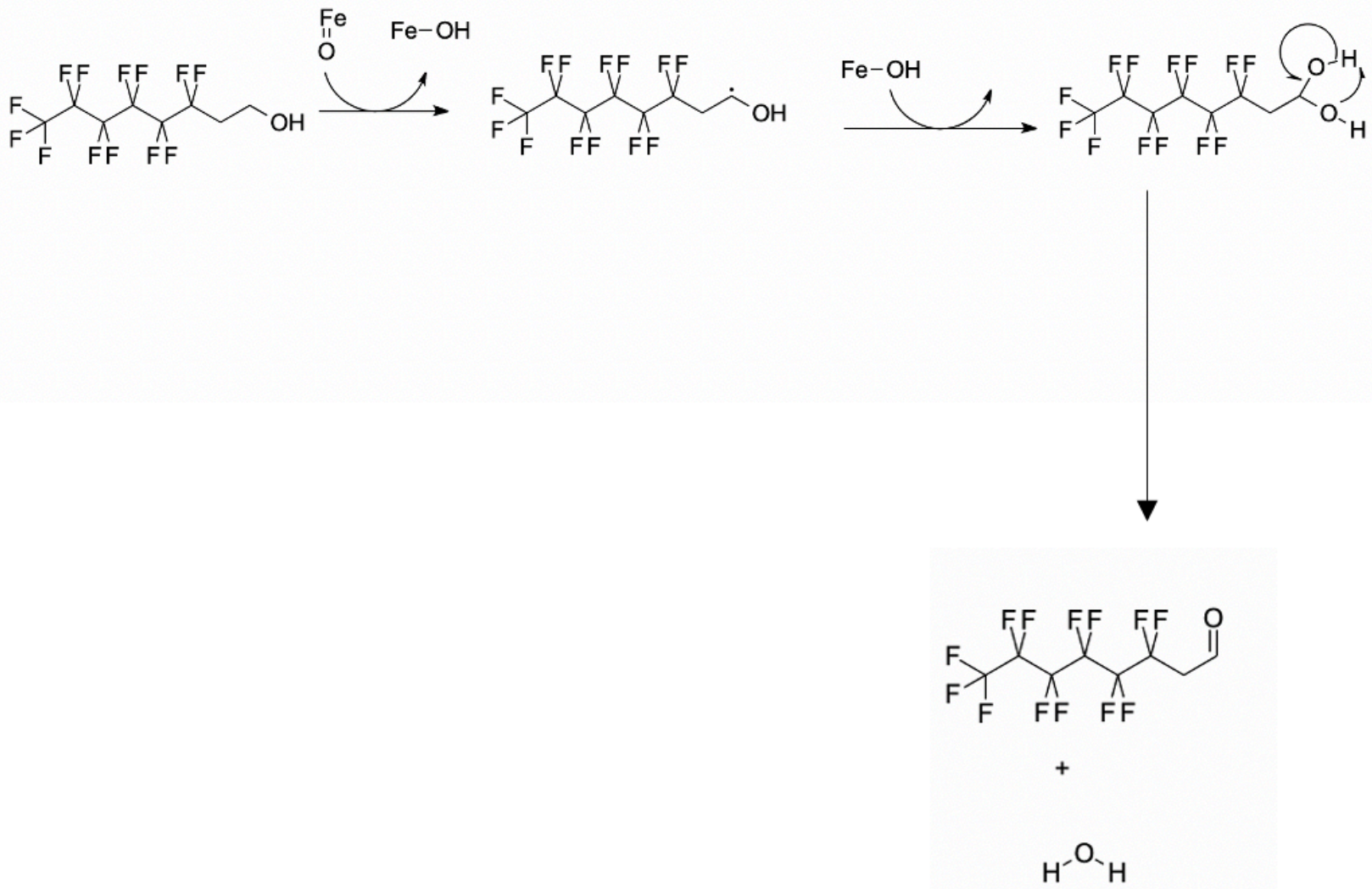

Figure 1.3 Phase I oxidation of 6:2 FTOH to 6:2 FTAL mediated by CYP in the presence of $\mathrm{NADPH}$ and diatomic oxygen.

Human CYPs are primarily found in the membrane of the mitochondria or the endoplasmic reticulum ${ }^{[13]}$. They are found at varying concentrations in different organs of the human body ${ }^{[14]}$. The liver is the primary organ for the first-pass metabolism, and is targeted for enterohepatic recirculation (reabsorption of the xenobiotic and/or its metabolites from external tissues, back into the liver) and thus contains a comprehensive assortment of CYP isoforms [14][15]. CYPs are found in specific cells of organs besides the liver. The lungs, kidney and intestinal tract all contain select cells which express a variety of CYPs ${ }^{[16][17][18]}$. The GI tract, although primarily used for absorption of compounds, is highly expressive for CYP $2 \mathrm{C} 9$ and the 
CYP 3A subfamily ${ }^{[16]}$. The lungs contain club cells, found in the bronchioles, which contain CYPs on their endoplasmic reticulum ${ }^{[19]}$. The kidney is expressive for only $2 \mathrm{~B} 6$ and $3 \mathrm{~A} 5$ with regards to the CYP family 1-3 in humans ${ }^{[20][21]}$. The presence of CYPs in various organs could lead to extrahepatic toxicity through phase I activation of toxicants. In figure 1.4 , the relative concentrations of CYPs in the human liver is illustrated. CYP 3A is a subfamily of CYPs that are abundant in hepatocyte, lungs, kidneys and GI tract. CYP $3 \mathrm{~A}$ accounts for over $80 \%$ of the activation of prodrugs in the market. This can be attributed to the subfamily $3 \mathrm{~A}$ being the most expressed CYPs in the liver and GI tract, as well as the large active sites of the members of the family (CYP 3A4).
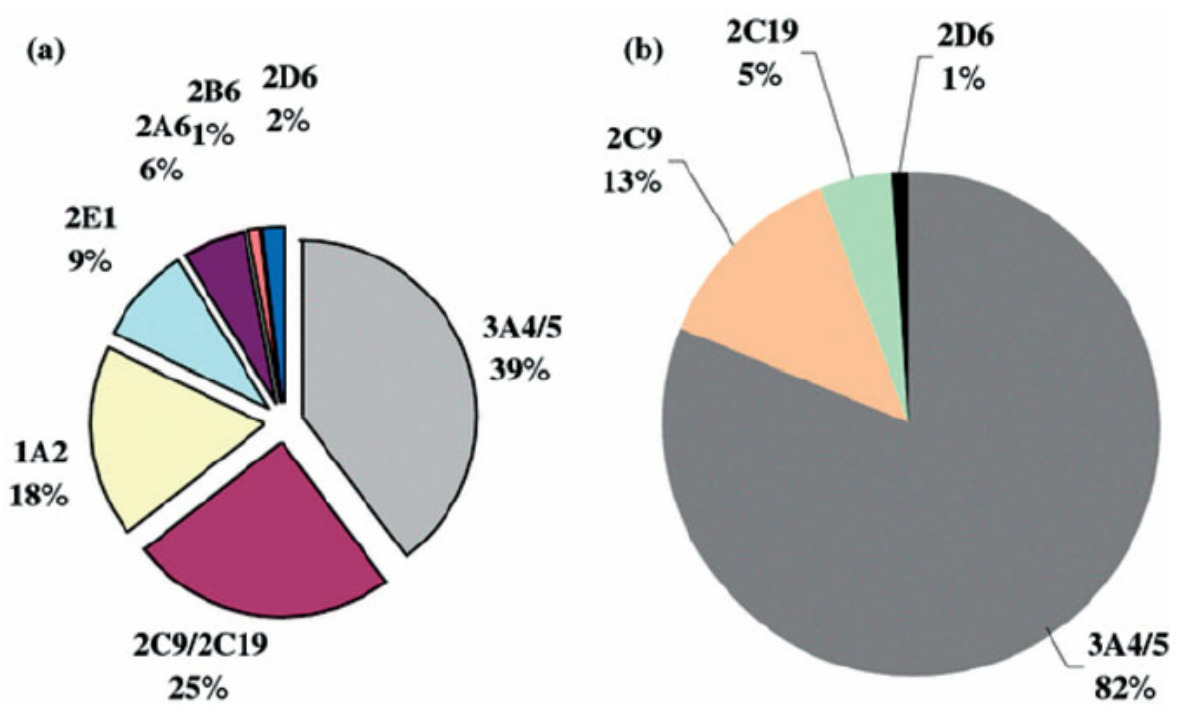

Figure 1.4: Distribution of CYPs in the human liver (a) and small intestine (b) ${ }^{[22]}$.

\subsection{Organofluorine compounds}

Organofluorines are compounds with a sigma bond between carbon and fluorine. The C-F bond is one of the strongest sigma bonds found in organic compounds ${ }^{[23]}$. The significant difference in electronegativity between the fluorine (4.0) and the carbon (2.5) yields a magnetic dipole moment for the sigma bond, with partial charges on the fluorine $\left(\delta^{-}\right)$and carbon $\left(\delta^{+}\right)^{[24]}$. 
Organofluorines can be further separated into different sub-categories of compounds. Fluorocarbons, fluorocarbenes and hydrofluorocarbons are examples of organofluorines. Fluorocarbons consist purely of the aforementioned C-F sigma bonds. Fluorocarbenes are a fluorinated version of traditional carbenes. An example being the difluorocarbene $\left(\mathrm{CF}_{2}\right)$, a variant of carbene $\left(\mathrm{CH}_{2}\right)$. Hydrofluorocarbons (HFCs) consist of a centralized carbon skeleton bonded to both hydrogen and fluorine. Hydrofluorocarbons are a prevalent variant of organofluorines. They are primarily used for refrigerant, air conditioning and insulating foam ${ }^{[26]}$. They used in place of phased out compounds like chlorofluorocarbons (CFCs) and hydrochlorofluorocarbons (HCFCs) ${ }^{[24]}$. HFCs, like 1,1,1,2-tetrafluoroethane, are commonly used in refrigerants. They consist of both $\mathrm{C}-\mathrm{H}, \mathrm{C}$-heteroatom and $\mathrm{C}-\mathrm{F}$ bonds.

Two environmentally important organofluorines are poly- and per-fluoroalkyl substances (PFAS). The perfluoroalkyl variants have no C-H bonds. The only carbon bonds involve fluorine and other electronegative compounds $(\mathrm{O}, \mathrm{S}, \mathrm{Cl}$, etc). Perfluorooctanoic acid (PFOA) is an example of a perfluoroalkyl substance, with only C-F bonds and a carboxylic polar head group. Fluorotelomer alcohols (FTOHs) are an example of the polyfluoroalkyl class. Polyfluoroalkyl compounds are similar to perfluoroalkyl compounds with the only difference being the presence of $\mathrm{C}-\mathrm{H}$ bonds connecting the $\mathrm{C}-\mathrm{F}$ tail to the polar head group. PFAS are prevalent in industrial products centred around flame retardants, oil resistance and water-resistant materials ${ }^{[25]}$.

Firefighting foams are a common source of workplace PFAS contamination ${ }^{[26]}$. Fluorosurfactants have been used as firefighting foams since the early $1960 \mathrm{~s}{ }^{[26]}$. Their widespread use in commercial products is due to the long C-F tail which provides PFAS with increased chemical and thermal stability compared to their hydrocarbon homologues ${ }^{[27]}$. The non-polar nature of the saturated skeleton coupled with the polar head group provides the unique 
properties of hydrophobicity and lipophobicity ${ }^{[26][28]}$. This makes the PFAS suitable compounds for greaseproof materials, so consequently are common with kitchen tools and food packaging ${ }^{[31]}$. Microwaveable popcorn bags and non-stick kitchenware are some of the direct sources of human contamination of PFASs, with ingestion being a key route of exposure ${ }^{[29]}$.

The presence of a C-H bond in the polyfluoroalkyl variant makes it susceptible to biotic and abiotic degradation unlike its perfluoroalkyl counterparts ${ }^{[30]}$. The degradation of the polyfluoroalkyl compound takes a myriad of paths before it reaches the terminal perfluorinated products. The possible terminating compounds of metabolism for these polyfluorinated compounds (e.g., 6:2 FTOH) is the transformation into perfluorinated carboxylic acids (e.g., PFHxA and PFPeA) and to a shorter chain polyfluorinated carboxylic acid (5:3 FTA) ${ }^{[31]}$, as seen in figure 1.5. 


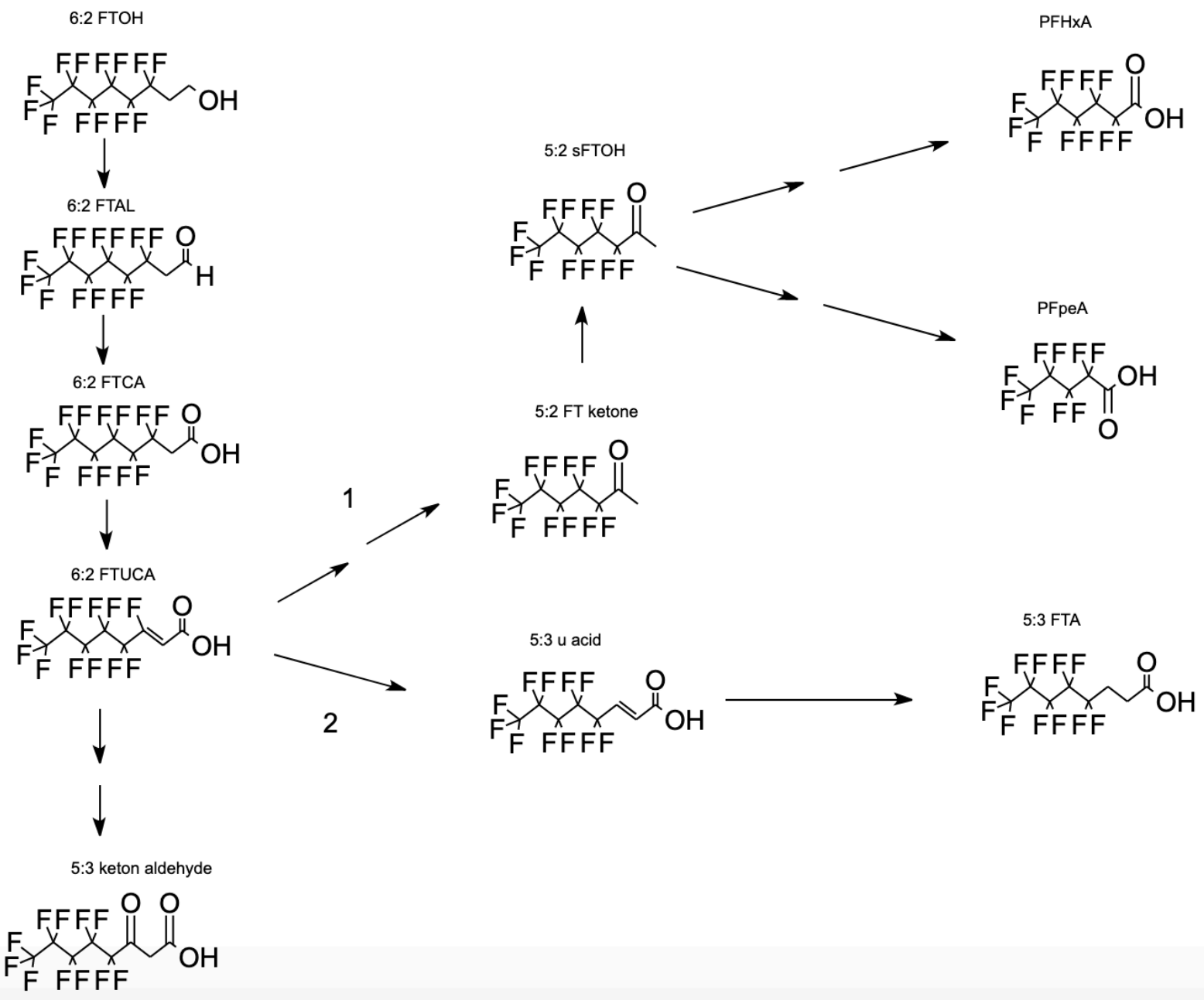

Figure 1.5: Possible biotransformation pathway for 6:2 $\mathrm{FTOH}^{[31]}$.

The perfluorinated compound could have the same number of carbons found in the fluorinated tail, 6:2 FTOH to PFHxA (6 carbons). The compound could also have a difference in the number of carbons found in the fluorinated tail, 6:2 FTOH to perfluoropentanoic acid (6 and 5 carbons respectively). The different possible pathways for the metabolism of 6:2 FTOH is shown in Fig 1.5. The lack of a single $\mathrm{C}-\mathrm{H}$ bond, coupled with the inactive $\mathrm{C}-\mathrm{F}$ bond, leads to bioaccumulation of the PFCAs, which is proportional to increasing fluorinated chain length ${ }^{[32][25]}$. Although PFCAs have a carboxylic acid group, which contain a nucleophilic -OH which should be susceptible for glucuronidation, phase II elimination of PFCAs has not been observed. 
As seen in Figure 1.5, the removal of these perfluoroalkyl substances alone will not limit their presence in the environment. Perfluoroalkyl substances are terminating metabolites of several polyfluorinated compounds. One major class of polyfluorinated compounds are FTOHs.

\subsection{Fluorotelomer alcohols and their transformation products}

The FTOHs are precursors of PFCAs ${ }^{[31]}$. They are a class of compounds with a fluorinated tail that can range from 6-10 carbons, and an alcohol head group. They are denoted by the nomenclature X:Y FTOH. "X" is the length of the nonpolar tail and "Y" the length of the non-fluorinated carbon linkage. Figure 1.6 is an image of 6:2 FTOH.

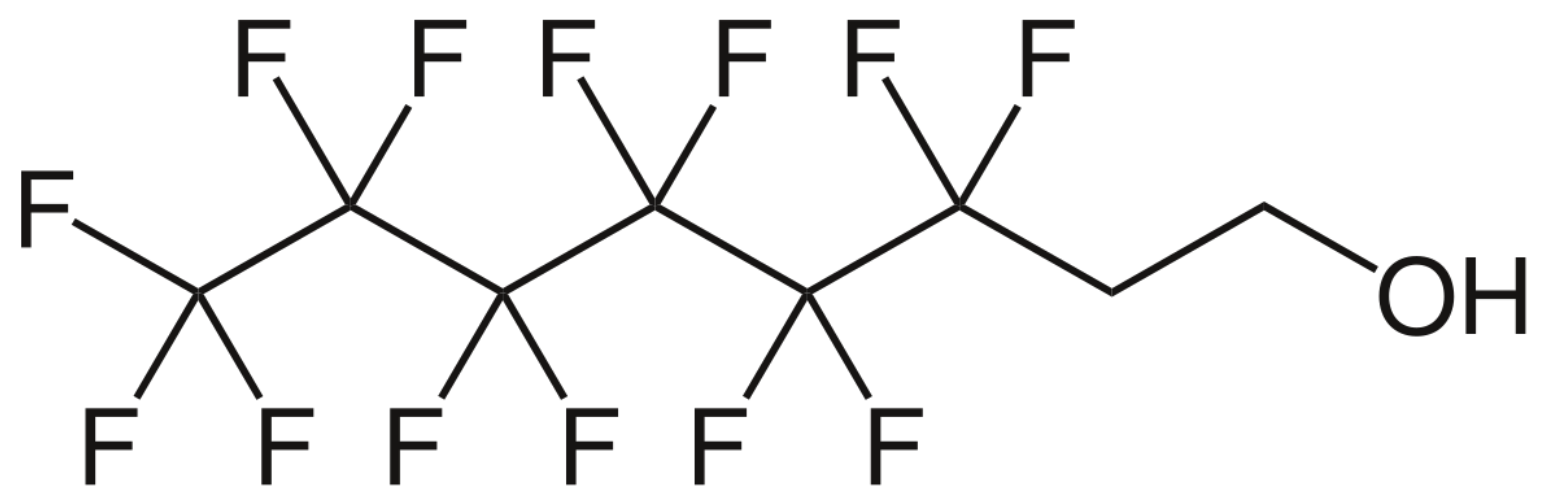

Figure 1.6: 6:2 Fluorotelomer Alcohol (FTOH)

The FTOHs are precursors to a host of toxic and bioaccumulative compounds like PFCAs

[31]. The 8:2 FTOH is a known precursor of PFOA, which has been shown to induce tumours in humans and mitochondrial toxicity in aquatic life (Zebrafish) ${ }^{[33][34][35]}$. Direct exposure to PFCAs has deemed the primary route of contamination. However, indirect exposure to precursors of PFCAs, like FTOHs, could be a significant source of PFCAs.

FTOHs can be synthesized through telomerization of tetrafluoroethylene monomer and pentafluoroethyl iodide telogen, forming an oligomer ${ }^{[36]}$. They can also be achieved through the metabolism or abiotic transformation of precursor PFAS like fluorotelomer acrylate and mono- / 
di-PAPs (polyfluorinated alkyl phosphates) which are used for the creation of fluorinated polymers ${ }^{[30]}$. FTOHs have been shown to metabolized from fluorotelomer acrylate in rainbow trout and human in-vitro liver assays ${ }^{[30]}$. Mono-/di-PAPs have also been shown to yield FTOHs in human liver, gut and faecal assays ${ }^{[37]}$. Due to FTOHs being a metabolite of known polymer reactants, they can be found in a variety of commercial products. Like PFCAs, they are found in greaseproof paper, firefighting foam (by-products) and as intermediates of polymer and surfactant synthesis ${ }^{[26][28][30]}$.

FTOHs exhibit similar chemical and physical characteristics to PFCAs. They both contain fluorinated tails that increase their lipophobicity ${ }^{[32]}$. FTOHs, however, are highly volatile, inversely proportional to the length of the fluorinated tail ${ }^{[38][39]}$. FTOHs are less polar than PFCAs with the same fluorinated carbon tail ${ }^{[40][41]}$. This is because, in environmental conditions, PFCAs are in an ionic form ${ }^{[41]}$. FTOHs are more volatile than PFCAs that share the same fluorinated carbon tail ${ }^{[38][39]}$. This volatility leads to atmospheric contamination, just like their respective PFCA ${ }^{[42][43][44]}$. In addition, FTOHs are often found in household dust ${ }^{[45]}$. The main environmental concerns for FTOHs have been their transformation and subsequent bioaccumulation of PFCAs.

Fluorotelomer carboxylic acids (FTCAs) and fluorotelomer aldehydes (FTALs) are two intermediates in FTOH transformation into PFCAs. The creation of these two compounds could increase the intrinsic toxicity of FTOHs. FTCAs and FTALs, formed from the metabolism of FTOH, as seen in Table 1.5, are 10,000x more toxic than their respective PFCAs in aquatic organisms ${ }^{[46]}$. Unsaturated versions of FTCAs (FTUCA) and FTALs (FTUAL) are also known metabolites that must be accounted for when qualifying the toxicity of FTOHs due to the presence of a double bond that is primed for nucleophilic attacks. The double bond in the 
unsaturated variants creates an electrophilic carbon that can be attacked by proteins, thus changing the function of these proteins. This will lead to an increase in toxicity.

Research has also provided evidence that FTOHs are a source of PFCAs to remote Arctic regions ${ }^{[47]}$. PFCAs (e.g., PFOA and PFNA) have been identified in the Arctic waters and other large bodies of water around the world ${ }^{[47]}$. PFCAs have been detected at local wastewater plants in North America, to animals and ice samples in the polar regions ${ }^{[49][50][51][52][53]}$. They are transported through currents to far-reaching points on the globe. Waste dumps in the North Atlantic and the North Pacific oceans feed into the contamination of the Arctic with PFCAs ${ }^{[49]}$. These strong polar acids leech away from commercial products in landfill and settle in the world's oceans. The solubility of PFAS is inversely proportional to the length of the fluorinated carbon tail, which leads to increased concern over the use of shorter chain PFAS as a suitable replacement for the $\geq 8$ chain length variants ${ }^{[41]}$. This increased solubility drives the leeching of PFAS into water bodies, increasing aquatic contamination. In remote regions of the Arctic contamination by PFAS has been shown in water bodies ${ }^{[47]}$. Transportation of leeched PFASs from waste dumps, through oceanic current, is the culprit for these water bodies contaminations ${ }^{[49]}$. However, PFCAs have been quantified in high arctic ice caps ${ }^{[53]}$. PFCAs have low vapour pressures and in environmental conditions exist as anions ${ }^{[40]}$. This points away from long-range atmospheric transportation in the gas phase. Without a connection to a contaminated water body, PFCA contamination has been found in these isolated ice caps ${ }^{[53]}$. These arctic contaminations are driven by volatilized FTOHs, which undergo abiotic oxidation to PFCAs. Volatile FTOHs are transported through the atmosphere. During the journey, these FTOHs are oxidized by the reaction with free radical $\mathrm{OH}$, in the presence of $\mathrm{O}_{2}$, to form the FTAL as seen in Figure $1.7^{[54]}$. 

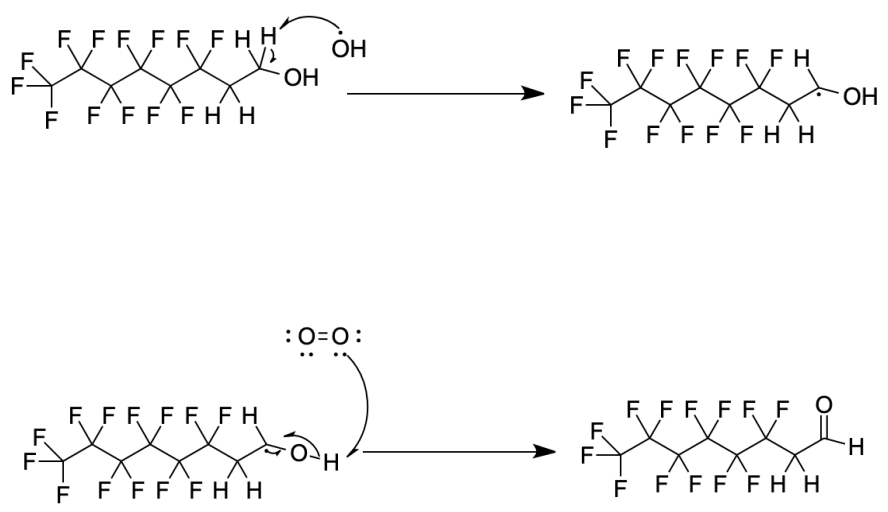

Figure 1.7: Reaction of 6:2 FTOH with free radical $\mathrm{OH}$ and diatomic oxygen to form 6:2 FTOH.

Further reactions with free radical $\mathrm{OH}$ lead to the formation of acyl peroxy radicals. Further reaction with $\mathrm{NO}, \mathrm{NO}_{2}, \mathrm{HO}_{2}$ or other peroxy radicals leads to the creation of terminating compounds like PFCAs and other persistent products ${ }^{[54]}$.

Biotic transformation of FTOHs begins with the metabolism of the FTAL ${ }^{[11]}$. Several studies have confirmed the biotransformation pathway of 8:2 FTOH to identify the CYPs responsible for the metabolism. In-vitro assays have confirmed the enzyme responsible for 8:2 FTOH phase I metabolism to 8:2 FTAL in humans is CYP $2 \mathrm{C} 19^{[55]}$. This was shown using data from pooled human liver microsomes and cytosol, along with an assay containing recombinant human CYP 2C19. Several purified CYP isoforms (CYP1A1, 1A2, 1B1, 2A6, 2B6, 2C8, 2C9,2C19, 2D6, 2E1, and 3A4) were individually incubated with 8:2 FTOH. Metabolically important CYPs were determined by the $15 \%$ loss of $8: 2 \mathrm{FTOH}$ over 4 hours. Only the human recombinant CYP 2C19 was deemed metabolically significant ${ }^{[55]}$. The metabolic importance of CYP 2C19 was confirmed by selective inhibition of CYP 2C19 in the human liver microsome 
and cytosol assay. Omeprazole was used as a selective inhibitor. The addition of omeprazole reduced the loss of 8:2 FTOH in the microsome and cytosol assays almost completely ${ }^{[55]}$.

In absence of an inhibitor, the 6:2 FTOH metabolizes to the 6:2 FTAL, which then undergoes dehydrogenation to form the unsaturated aldehyde, 6:2 FTUAL ${ }^{[31]}$. It can also metabolize to form 6:2 FTCA ${ }^{[31]}$. The 6:2 FTUAL is metabolized by phase I enzymes to form the unsaturated carboxylic acid, 6:2 FTUCA, as well as other aldehyde isomers, shown in Figure 1.5. Five major terminating metabolites are formed: PFHxA, PFHpA, 5-2s-FTOH, 5:3 Acid, etc. The terminating metabolites are shown in Figure 1.5.

Elimination of 6:2 FTOH also occurs through phase II conjugation as seen in Figure 1.5. Once metabolized, the unsaturated aldehyde (FTUAL) and carboxylic acid (FTUCA) provides an additional site for phase II conjugation reactions, specifically with the antioxidant glutathione. In addition, the highly electrophilic beta-carbon in FTUAL, when not conjugated to glutathione ${ }^{[56] \text {, }}$ has been shown to covalently modify proteins, contributing to toxicity several folds more than the PFCAs in human liver epithelial cells ${ }^{[57][58]}$. Further understanding about how FTOH metabolism relates to the toxicity of PFCAs and other intermediate metabolites such as the FTUAL, is warranted, especially since FTOH toxicity may arise from its CYP metabolism to bioactive products.

\section{$1.4 \quad \underline{\text { Toxicity }}$}

Toxicity is the ability of a compound to yield an adverse effect. It can be measured as an effect on a particular organ, region of the organism, or an effect on the whole organism. Toxicity is grouped into two categories: acute and chronic. Acute toxicity is the amount of immediate exposure to a toxicant that causes severe/lethal effects. This is typically quantified by the $\mathrm{LD}_{50}$ value. The $\mathrm{LD}_{50}$ stands for the median lethal dose and denotes the amount of a substance needed 
to kill $50 \%$ of a tested group over a fixed time. Chronic toxicity is the constant exposure to a non-acute amount of toxicant that can yield future adverse effects. The intrinsic toxicity of a given material stems from the relationship between the acute and chronic amount. The severity of a toxicant is affected by how little of the material yields the toxic effect. It is also affected by how frequent and easy it is to contaminate the organism. Toxicants can also be grouped based on how the compound facilitates the adverse effect. These groups are chemical, physical, radiative, biological, and behavioural toxicity. The three most common toxic groups are chemical, physical, and biological. Benzene, asbestos, and viruses are examples of chemical, physical and biological toxicants respectively.

The FTOHs and their metabolites are sorted into chemical toxicity. This is because their toxic effect is derived from chemical reactions with proteins and DNA or toxicity concerning non-covalent binding to receptors. There are few major pathways on the toxicity of FTOHs and their metabolites. Nucleophilic attacks by proteins and DNA on the FTCA and FTALs could lead to an increase in toxicity ${ }^{[57][58]}$. The creation of an electrophilic carbon in the unsaturated FTCA and FTAL creates an additional position for nucleophilic attacks. The toxicity of PFAS like PFOA and PFOS have been widely investigated. PFOA has also been shown to promote tumour growth in Sprague-Dawley rats' assays ${ }^{[33]}$. PFOA is a well-known tumour promoting compound and developmental toxicant ${ }^{[35]}$. PFOA induces adenomas of the liver, testis (Leydig cell tumours), and pancreas (acinar cell tumours) like others through PPAR $\alpha$ receptor agonist. The mode of action for tumour promotion in rodents for PFOA has been reported ${ }^{[33]}$. PFOA activates the PPAR $\alpha$ receptor. This results in the up-regulation of cell growth gene expression in the liver, resulting in cell proliferation ${ }^{[35][33]}$. Human in-vitro studies focused on the PFOA's effect on liver cells revealed that cell death due to oxidative stress is the leading cause of toxicity [34]. 
PFOA leads to the creation of radical oxygen species (ROS) which leads to apoptosis in human hepatoblastoma HepG2 cells ${ }^{[34]}$. Perfluorohexanoic acid (PFHxA), a major terminating metabolite for 6:2 FTOH is not carcinogenic in rat or human in-vitro models. PFHxA did not create ROS in human in-vitro and did not affect human hepatoblastoma HepG2 cells ${ }^{[59]}$. No relationship between PFHxA and receptor toxicity has been found in either animal or human models. Within human liver epithelial cells, PFHxA showed reduced toxicity when compared to long PFCAs, having lower $\mathrm{EC}_{50}$ values ${ }^{[57]}$. The decreased toxicity has been suggested to be due to the shorter fluorocarbon tail compared to PFOA and higher chain length PFCAs, which leads to reduced bioaccumulation.

Research focusing on FTOH-mediated toxicity has centred on the 6:2, 8:2, 10:2 congeners, due to their commercial and industrial use. The 8:2 and 10:2 FTOH have been shown to exhibit developmental and reproductive toxicity in mice ${ }^{[60]} .6: 2 \mathrm{FTOH}$ has been shown to induce developmental toxicity in mice but only at a dosage that also induced maternal toxicity. This means that 6:2 FTOH is not a selective developmental toxicant as the adverse effect on the unborn child could be a side effect of the damage done to the mother ${ }^{[61]}$. The $6: 2$ and 8:2 FTOH has been shown to affect the hormonal concentration of testosterone and oestradiol in aquatic species (Zebrafish) ${ }^{[62]}$.

The FTOHs metabolize to bioactive intermediates, like FTCAs, FTUCAs, FTALs, and FTUALs. The FTCA and FTUCA are more toxic to aquatic wildlife than their PFCA counterpart. The three aquatic species examined within this study were Daphnia magna (planktonic crustacean), Chironomus tentans (freshwater invertebrate), and Lemna gibba (duckweed) ${ }^{[64]}$. Toxicity was determined by the timed survival and immobility of $D$. magna and C. tentans, with toxicity on the L. gibba based on frond number and dry biomass ${ }^{[64]}$. Within 
human liver epithelial cells, FTUCAs and FTCAs were more potent at reducing cell viability, having around $5 \mathrm{x}$ lower $\mathrm{EC}_{50}$ values than PFCAs of similar fluorinated chain length ${ }^{[58]}$. Both FTUCAs and FTCAs, like PFCAs, showed increased toxicity with the increase in the length of the carbon tail. By contrast, FTALs and FTUALs had an inverse relationship between their toxicity and the length of the carbon tail. Shorter chain length FTALs and FTUALs (e.g., 6:2) were more toxic, exhibiting a lower $\mathrm{EC}_{50}$ value than their longer counterparts (e.g., 8:2) ${ }^{[58]}$. The FTALs also showed a lower $\mathrm{EC}_{50}$ value than the corresponding PFCAs, FTCAs, and FTUCAs. The hypothesized mechanism of toxicity for the aldehyde metabolites (e.g., FTUALs) is through depletion of GSH and the subsequent formation of covalent protein adducts. Protein adducts may inhibit protein function and lead to increased toxicity, although the toxic mechanism is currently unknown ${ }^{[58]}$.

\subsection{PFAS regulation}

PFOA and Perfluorooctanesulfonic acid (PFOS) are key PFAS of concern due to their known toxicological effects on animals and humans. PFOA, as stated before, is a tumour promoting PFAS. PFOS, like PFOA, is a bioaccumulating compound. PFOS has been shown to affect the immune system in mice studies. Due to their environmental persistence and adverse effects on wildlife and humans, both have been included in annex categories within the Stockholm convention ${ }^{[65][66]}$.

The Stockholm Convention on persistent organic pollutants (POPs) is an international treaty surrounding the regulation of organic pollutants ${ }^{[67]}$. It came into effect in $2004^{[67]}$. The treaty was signed by 152 founding countries ${ }^{[67]}$. A significant country of absence is the United States of America (USA). The USA signed the treaty in 2001 but have yet to ratify the treaty ${ }^{[67]}$.

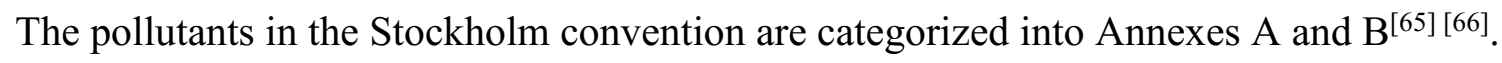


Included in annex A are compounds that involve countries that will seek to eliminate their use and production ${ }^{[65]}$. The precursors of these compounds are also eliminated from production. Annex B consists of compounds that the involving countries will seek to restrict their use and production $^{[66]}$. PFOA and its precursors are the only PFAS listed in annex A of the Stockholm convention. PFOS and its precursors are the only PFAS listed in annex B of the Stockholm convention. Currently, there are no new PFCAs under investigation for addition to the Stockholm convention. Perfluorohexane-1-sulfonic acid (PFHxS) has been recommended to the governing parties for inclusion into Annex A of the Stockholm convention. Sulfonic PFAS however do not metabolise from FTOHs.

The Stockholm convention seeks to constantly evaluate the effectiveness of the regulations. These evaluations are based on the regulations effect on ${ }^{[68]}$ :

1. The decrease in intentional and unintentional release of persistent organic pollutants in both annexes

2. The reduction in the release of annexed compounds from the current stockpile

3. The reduction of environmental concentrations of the annexed compounds.

Due to their evaluation parameters, a few widely used POPs have seen a vast reduction in use and some cases a complete elimination in their production and the use of their stockpiles. For example, chlordane is a compound in annex A of the Stockholm Convention ${ }^{[69]}$. Chlordane was widely used as a pesticide ${ }^{[68]}$. Due to the Stockholm convention and other internal environmental bodies, countries all over the world have ceased the use and production of Chlordane ${ }^{[69]}$. Chlordane is one of the 12 initial POPs banned by the Stockholm convention in 2001, along with the commonly known DDT ${ }^{[69]}$. 
USA's absence in the Stockholm convention gives the EPA and FDA the regulatory power over POPs at a federal level. Like the Stockholm convention, the key PFAS of note are PFOA and PFOS ${ }^{[70]}$ Unlike the Stockholm convention, particularly annex A compounds, there is no blanket ban on the use and production of individual PFAS. A key source of regulation for the EPA is the safety of drinking water ${ }^{[71]}$. As of February 2020, the EPA has outlined action plans for the clean-up, monitoring and reduction of PFAS in drinking water ${ }^{[71]}$. The restriction of PFOA in the USA is lagging the nations included in the Stockholm convention. There are no new updates on the definitive acts the EPA is laying out for further PFOA and PFOS regulation in drinking water. The current EPA guidelines are 70 parts per trillion of PFOA and PFOS in drinking water. All that has been released is an outline laying the groundwork for future regulation. PFOA is a major compound in these actions plans but there are other shorter PFAS like perfluorobutanesulfonic acid (PFBS) which are undergoing toxicity assessment for future regulations $^{[71]}$.

Along with drinking water, the EPA is also beginning to assess and develop regulations of the PFASs in biosolids ${ }^{[72]}$. Biosolids are solid organic matter extracted from sewage treatment. There are regulations of pathogens and metals and nutrients in the application of biosolids for agricultural processes but there is no PFAS content regulation. PFAS in biosolids has been shown to feed into the underlying water body as well as grazing animals. This leads to an additional source of human contamination ${ }^{[72]}$.

Given that the EPA is in the early stages of PFAS environmental risk assessment, most of the key changes for the reduction of PFAS contamination stems from non-government binding agreements. Shorter chain PFAS $(\mathrm{C}<=6)$ have been voluntarily phased out from food packaging manufacturing by major production companies based in the USA ${ }^{[73]}$. This is a step in the right 
direction in the elimination of shorter chain FTALs and FTUAL, which could prove vastly more toxic than their longer-chain counterpart.

In contrast to the USA, Canada is one of the signatories of the Stockholm convention. This means that there is a national ban on the production and use of PFOA and its salt precursors, as well as the restriction on the production and use of PFOS. Canada has expanded the regulations placed on PFOA to other PFAS. In 2006, Canada enacted the "Action Plan for the Assessment and Management of Perfluorinated Carboxylic Acids and their Precursors" [74]. This prohibits the manufacturing, sale, import of four fluorotelomer based substances, none of which are FTOHs. The regulations however do not prohibit the acquisition of goods from foreign countries that contain fluorotelomer based compounds. Shorter chain PFAS has not yet been placed under any regulations. The monitoring of the FTOHs is performed under the umbrella monitoring initiative started by Government Canada to track the environmental concentrations of PFCAs and their precursors. The monitoring program covers aquatic, air, sediment, and terrestrial biota, wastewater and biosolids. As of October 2016, the Prohibition of Certain Toxic Substances Regulations included PFOA and its precursors, long-chain PFCAs and their precursors, along with PFOS and its precursors ${ }^{[74]}$. Government regulation of shorter chain FTOHs (carbon tail $<\mathrm{C} 8$ ) has not been formally enacted. However, due to the monitoring of PFCAs and their precursors, Canada has issued a notice of intent to approach PFAS as a broad range of environmentally significant chemicals as of February $2021^{[75]}$. This allows for the inclusion of shorter chained PFASs in environmental regulations moving forward. 


\subsection{CYP Identification}

In vitro assays using extracted biological subcellular fractions are used to determine active enzymes in a catalyzed reaction, and models the reactions occurring in a specific organ. The same information can also be collected using extracted cells localized to an organ or tissue. These assays are useful in acquiring quick and reliable information about the fate of compounds in an organism. However, the true fate of the compound once introduced to the body could vary as in-vitro assays cannot fully replicate all the organism's internal functions.

The choice for a suitable in vitro fraction was determined by the organ that most likely to metabolize the 6:2 FTOH. In humans and wildlife, the liver is the primary processing organ for the first-pass metabolism of xenobiotics ${ }^{[76]}$. In humans, the major route of PFAS contamination is exposure through ingestion and inhalation of indoor air, since most human activity occurs indoors ${ }^{[77][78]}$. In the case of inhalation, compounds that enter the body through this route will be routed to the liver after being absorbed from the GI tract (Figure 1.8). Once in the gut, the 6:2 FTOH is transported to the liver through the portal vein, where metabolism may be initiated. However, FTOHs and their metabolites have also been found in the blood, umbilical cord blood and breast milk ${ }^{[33][52][79]}$. This indicates that not all the $6: 2 \mathrm{FTOH}$ is metabolized in the liver. There is the possibility of transportation to varying organs in the body, in which metabolism could activate the toxicant metabolites and induce extrahepatic toxicity. Once in the liver, the $6: 2$ FTOH will begin metabolism due to the liver's large concentration of CYPs ${ }^{[22]}$. If the $6: 2 \mathrm{FTOH}$ is not metabolized by CYP, it could be conjugated with a glucuronic acid or sulfate group by phase II enzymes. This increases the size and polarity of the 6:2 FTOH and it is removed from the liver into the GI tract through the bile, or into the kidney for renal excretion. The 6:2 FTOHconjugates in the GI tract can undergo hydrolysis, facilitated by intestinal bacteria or 
carboxylesterases, to revert to 6:2 FTOH. The 6:2 FTOH will once again be absorbed into the vascular system and transported to the liver for further metabolism, in a process called enterohepatic recirculation.

In addition to ingestion of 6:2 FTOH, inhalation could be another major route of exposure given its volatility. The FTOHs are highly volatile compounds as seen from their migration to remote arctic regions ${ }^{[47]}$. PFAS in cosmetic materials could also provide a significant source of dermal exposure ${ }^{[80][81]}$. For inhalation and dermal exposure of 6:2 FTOH, the first-pass transformation by uptake from the portal vein into the liver is not as accessible compared to exposure by ingestion. 


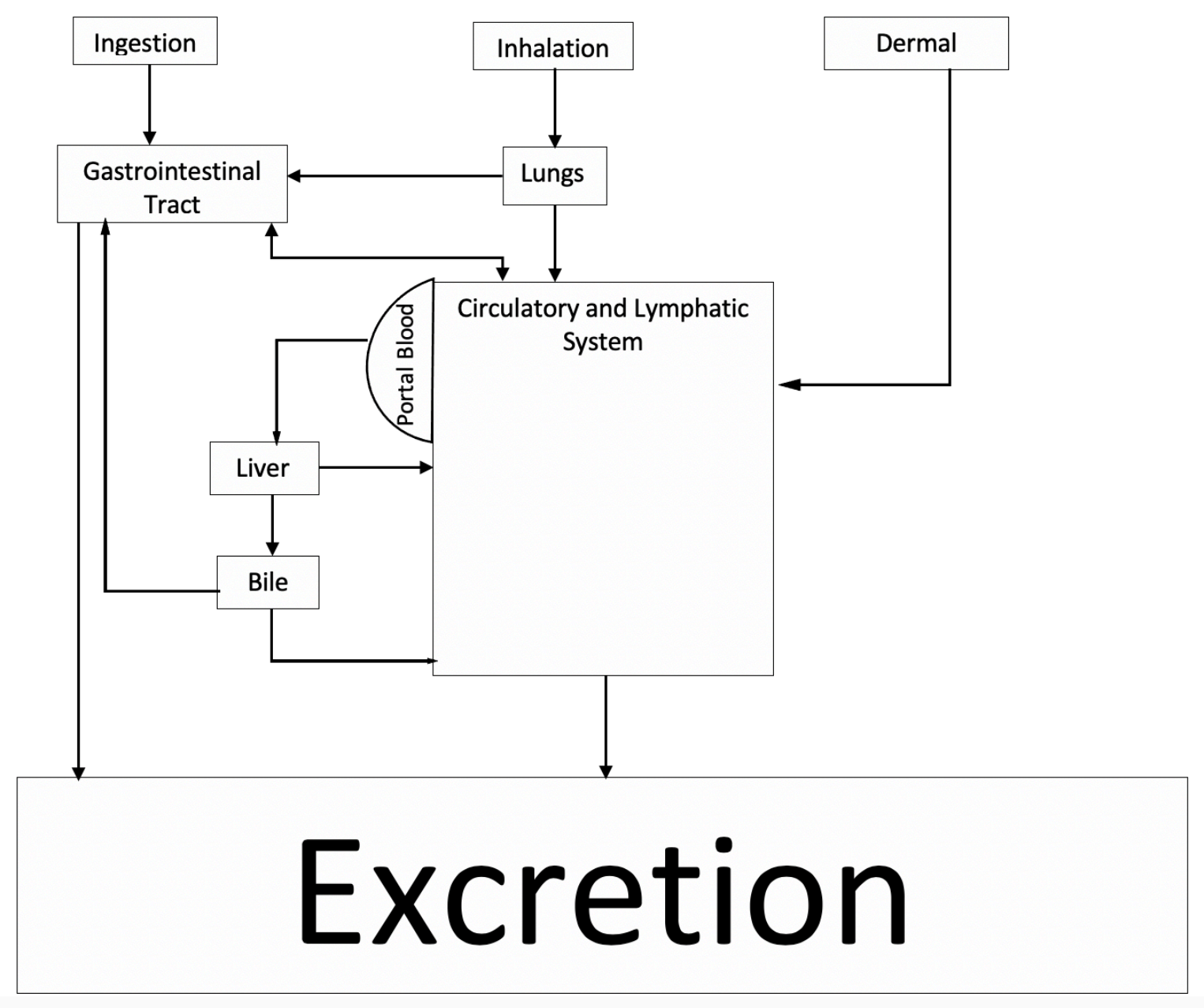

Figure 1.8: Primary route of exposure (ingestion, inhalation and dermal) for 6:2 FTOH and their transportation in the human body.

If 6:2 FTOH was absorbed after dermal or inhalation exposure, it will most likely enter the bloodstream and begin circulation throughout the body. Alternatively, it could also be absorbed and metabolized by extra-hepatic cells that may contain CYP. Although the concentration of CYPs in other organs is much smaller than that of the liver, certain CYPSs are still significantly expressed in non-hepatic organs. For example, CYP 2E1 is shown to be 
expressed in Club cells that inhabit selective regions of the lungs ${ }^{[83]}$. Therefore, 6:2 FTOH could be metabolized within this organ after inhalation ${ }^{[82]}$.

Given that the major reported route of 6:2 FTOH exposure is ingestion and inhalation, the liver is often chosen as the organ in vitro assays. A subcellular fraction extracted from the liver called microsomes is used for CYP mediated metabolism. Microsomes consists of pieces of the endoplasmic reticulum and are concentrated sources for CYPs ${ }^{[84]}$. Liver microsomes can be obtained through a four-step process as shown in Figure 1.9.

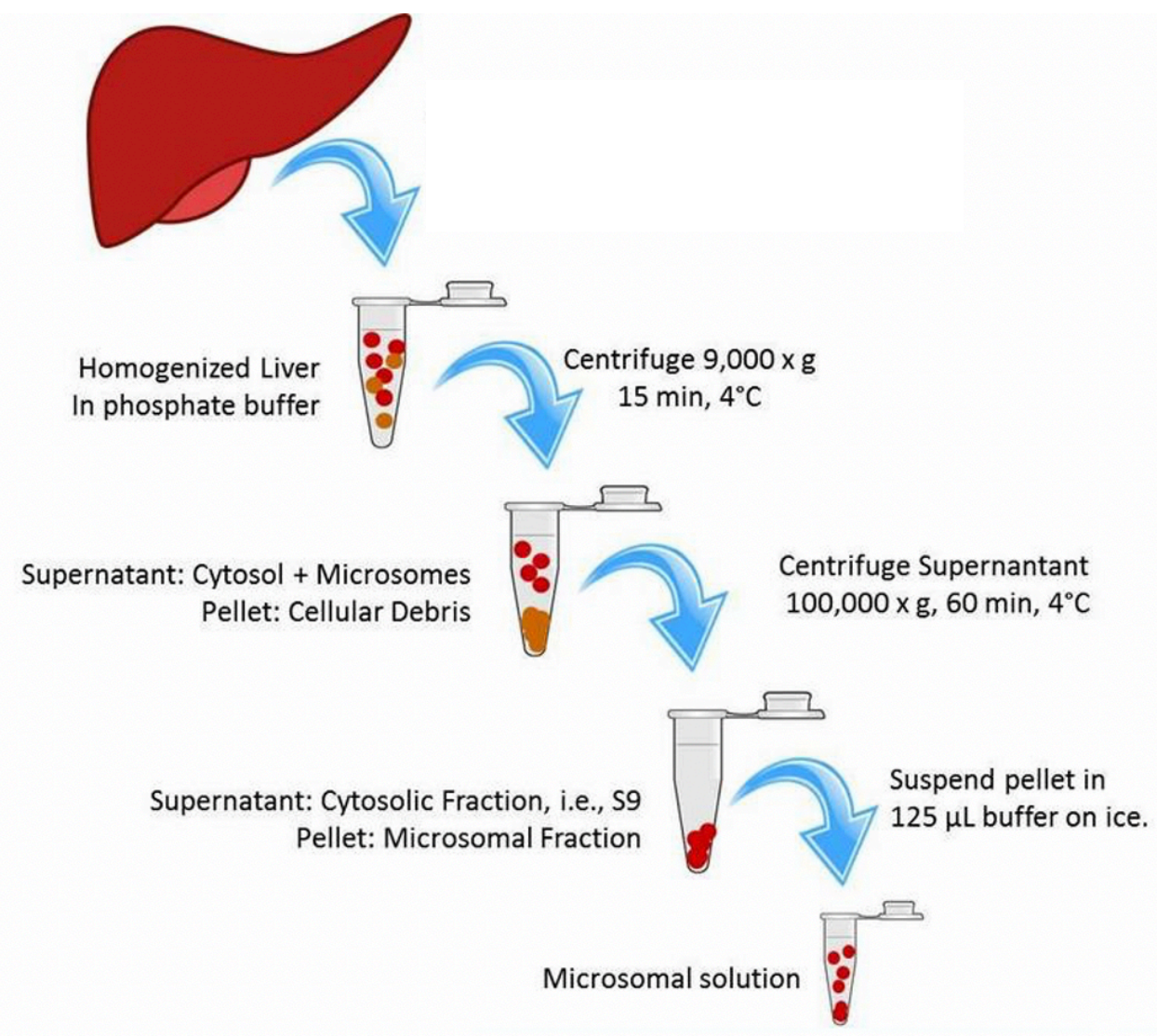

Figure 1.9: Procedure for the preparation of liver microsomes ${ }^{[85]}$. 
Incubation of 6:2 FTOH with liver microsomes is a suitable in vitro assay as the liver contains a large concentration of viable CYPs known to be responsible for xenobiotic metabolism; microsomes are commonly used to test for CYP activity in many pharmacological and toxicological metabolic studies based around phase I metabolism ${ }^{[11]}$. To determine the CYP responsible for metabolizing a specific substrate, microsomes are incubated with the substrate in question, with and without a specific CYP inhibitor that can suppress the rate of reaction. The uninhibited control is first used to acquire a standard reaction rate. If a CYP is responsible for metabolizing the substrate, adding a specific inhibitor of that CYP will suppress the reaction rate and should lead to a decrease in reaction rate proportionally to an increase in the concentrations of the inhibitor.

\subsection{Enzyme Kinetics}

Once the CYP has been identified, a Michaelis-Menten curve is usually generated for the noninhibited and inhibited assay. Michaelis- Menten curve provides two important constants, $\mathrm{V}_{\max }$ and $K_{\mathrm{M}}$, that are kinetics parameters that determine the efficiency of an enzymatic reaction. The $\mathrm{V}_{\max }$ denotes how the upper limit of the reaction rate of a saturated enzyme incubation. $K_{\mathrm{M}}$ denotes the affinity of the substrate to the enzyme binding site. The Michaelis-Menten curve is the relationship between the reaction rate (v), and the substrate concentration ([S]) of a given 
enzymatic reaction. The general mechanism of an enzymatic reaction is shown in Figure 1.10.

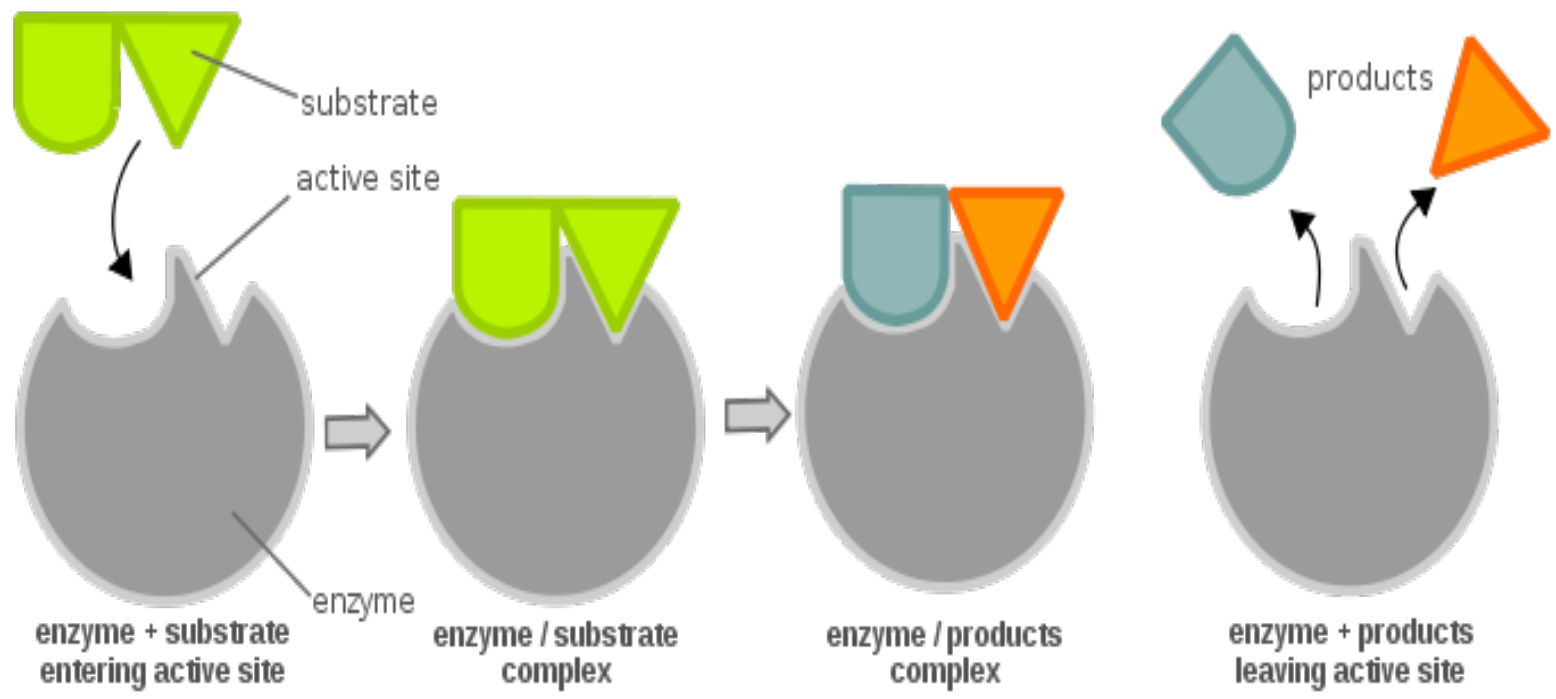

Figure 1.10: Illustration of an enzymatic reaction ${ }^{[86]}$.

Through the illustrated mechanism, the kinetic equation for an enzymatic reaction is as follows:

$$
\begin{aligned}
& \mathrm{E}+\mathrm{S} \stackrel{k_{1}}{\leftrightarrows} \mathrm{ES} \stackrel{k_{c a t}}{\rightarrow} \mathrm{E}+\mathrm{P} \\
& k_{-1}
\end{aligned}
$$

E, S, ES and P represent the enzyme, substrate, enzyme-substrate complex, and product respectively. The three reaction constants $\mathrm{k}_{1}, \mathrm{k}_{-1}, \mathrm{k}_{\mathrm{cat}}$, represent the forward rate, reversible rate, and catalytic rate constant respectively. The rate constant $\mathrm{k}_{1}$ and $\mathrm{k}_{-1}$ are assumed the same, resulting in an equilibrium between the formation of the enzyme-substrate complex and the separation of the complex. Given the concentration of the enzyme ([E]), is significantly lower than that of the substrate ([S]), the rate of product formation is determined to be: 


$$
\mathrm{v}=\mathrm{k}_{\mathrm{cat}}[\mathrm{E}]_{0} \frac{[\mathrm{S}]}{\mathrm{K}_{\mathrm{M}}+[\mathrm{S}]}
$$

$[E]_{0}$ represents the initial concentration of the enzyme. $K_{M}$ represents the concentration of substrate at which the reaction rate is half the maximum.

From the equation, we can see that if the substrate used to initiate the reaction is significantly higher than the $\mathrm{K}_{\mathrm{M}}$, the reaction rate will approach $\mathrm{k}_{\mathrm{cat}}[\mathrm{E}]_{0}$, also known as the maximum reaction rate $\left(\mathrm{V}_{\max }\right)$. This is seen in the Michaelis-Menten curve in Figure 1.11.

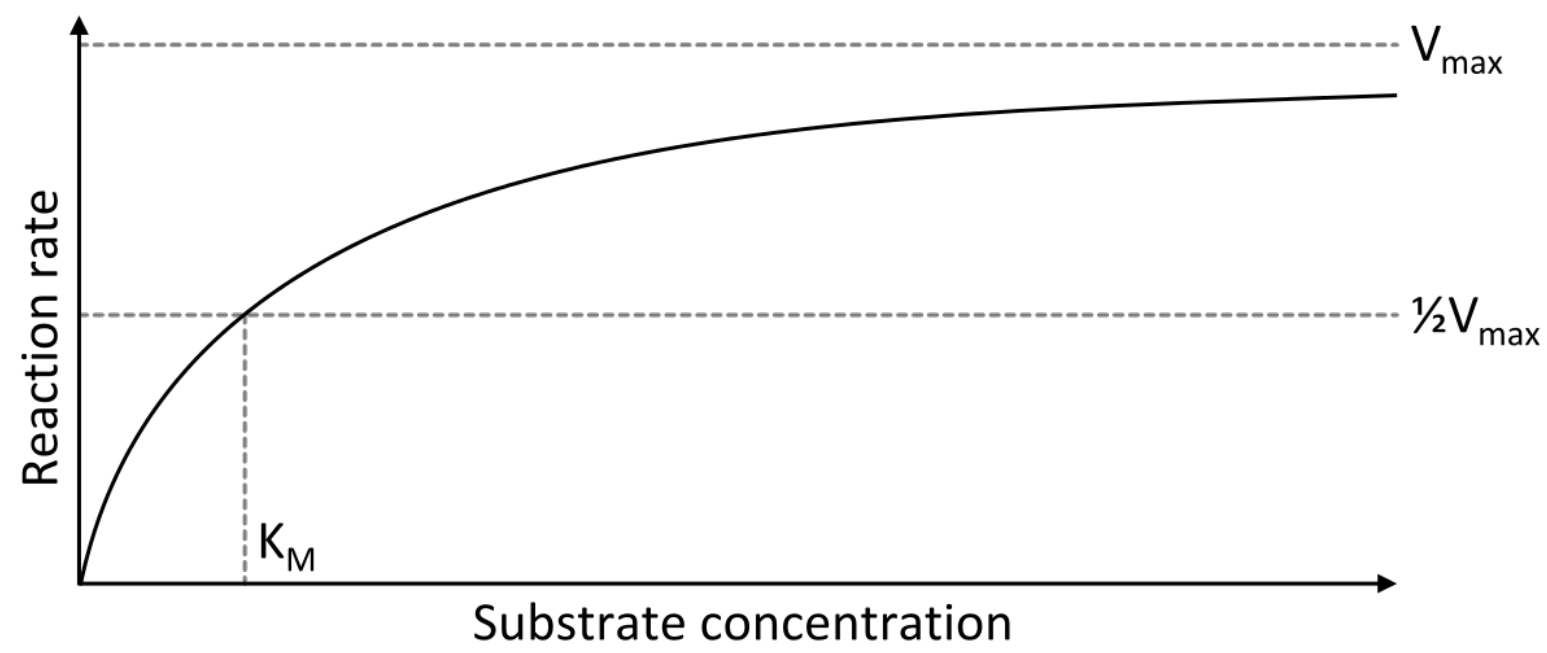

Figure 1.11: An illustration of a Michaelis-Menten curve with the constants $K_{M}$ and $v_{\max }$. As the substrate concentration increases, we approach a plateau zone at the $V_{\max }$. If the substrate is significantly smaller than the $\mathrm{K}_{\mathrm{M}}$, the reaction rate progresses at a linear rate of $\mathrm{k}_{\mathrm{cat}}[\mathrm{E}]_{0} \frac{[\mathrm{S}]}{\mathrm{K}_{\mathrm{M}}}$. This is known as the linear zone. Considering that $\mathrm{k}_{\mathrm{cat}}[\mathrm{E}]_{0}$ is the $\mathrm{V}_{\max }$, the rate for an enzymatic reaction can be expressed as:

$$
\mathrm{V}=\mathrm{V}_{\max } \frac{[\mathrm{S}]}{\mathrm{K}_{\mathrm{M}}+[\mathrm{S}]}
$$


If a substrate-enzyme reaction produces a low $K_{\mathrm{M}}$, it symbolizes a high bonding affinity between the enzyme and the substrate as it does not require a large concentration of the substrate to achieve half the maximum rate. Likewise, a high $K_{\mathrm{M}}$ value denotes a low binding affinity. The Michaelis-Menten curve provides the constants that determine how efficient an enzyme is at catalysing a reaction. The efficiency of an enzyme is based on its turnover rate. The turnover rate of an enzyme describes how quickly the enzyme binds to the substrate and how quickly it produces and releases the product. If the reaction is thermodynamically favoured regardless of the use of an enzyme, the enzyme does not affect the theoretical yield of the reaction. The reaction will produce the same number of products giving enough time. A high $\mathrm{V}_{\max }$ will lead to a more efficient enzyme as the product is produced faster. A lower $K_{\mathrm{M}}$ will also lead to a more efficient enzyme as the reactants bind quicker. The turnover rate, also known as the metabolic clearance $\left(\mathrm{CL}_{\text {int }}\right)$, is the ratio $\mathrm{V}_{\max } / \mathrm{K}_{\mathrm{M}}$. A higher $\mathrm{CL}_{\text {int }}$ when comparing all CYPs will determine the CYP primarily responsible for the metabolism of a substrate.

The $\mathrm{CL}_{\text {int }}$ is a specific way to determine the enzymes responsible for the metabolism of a substrate. Since we are working with microsomes, which contain many CYPs, increased support that a specific CYP is turning over the 6:2 FTOH can be gained by using selective inhibitors. An inhibitor is any compound that reduces the rate of a reaction. For enzymatic reactions, there are several ways by which an inhibitor reduces the reaction rate. As stated, before there are three major compounds/molecules involved in the production of the final product. The inhibitor can interfere with either the enzyme or the enzyme-substrate complex. There are three major types of inhibitors: competitive, uncompetitive, and non-competitive. Competitive inhibitors attach to the binding site, interfering with the formation of the ES complex. Due to the reduction of the ES complex, the $K_{\mathrm{M}}$ of the reaction is increased. The uncompetitive inhibitor binds to the ES 
complex, preventing the formation of the product. This hurts the formation of the ES complex due to Le Chatelier's principle, and thus the $K_{\mathrm{M}}$ is increased. The inhibitor-bound ES complex also reduces the production of the product and causes a reduction of the $\mathrm{V}_{\max }$. Non-competitive inhibitors bind to a different binding site on the enzyme, reducing the efficient production of the product. The use of a different binding site does not affect the production of the ES complex. This results in an unchanged $K_{\mathrm{M}}$. The use of a different binding site will affect the production of the product and cause a decrease in the $\mathrm{V}_{\max }$. Another lesser-known type of inhibition is suicide inhibition. Suicide inhibition involves the creation of an irreversible complex between the enzyme and an inhibitor. This complex eliminates the enzyme's ability to catalyse the reaction forever. This has a similar effect on the Michaelis Menten constants as the non-competitive inhibition. The is because the suicide inhibitor reduces the number of available enzymes. This only negatively affects the creation of the product and not the binding affinity. The $\mathrm{V}_{\mathrm{Max}}$ in a suicide inhibition will be decreased, while the $K_{\mathrm{M}}$ remains the same. Each of these inhibitions causes specific changes to the Michaelis-Menten curve which is illustrated in Figure 1.12. 


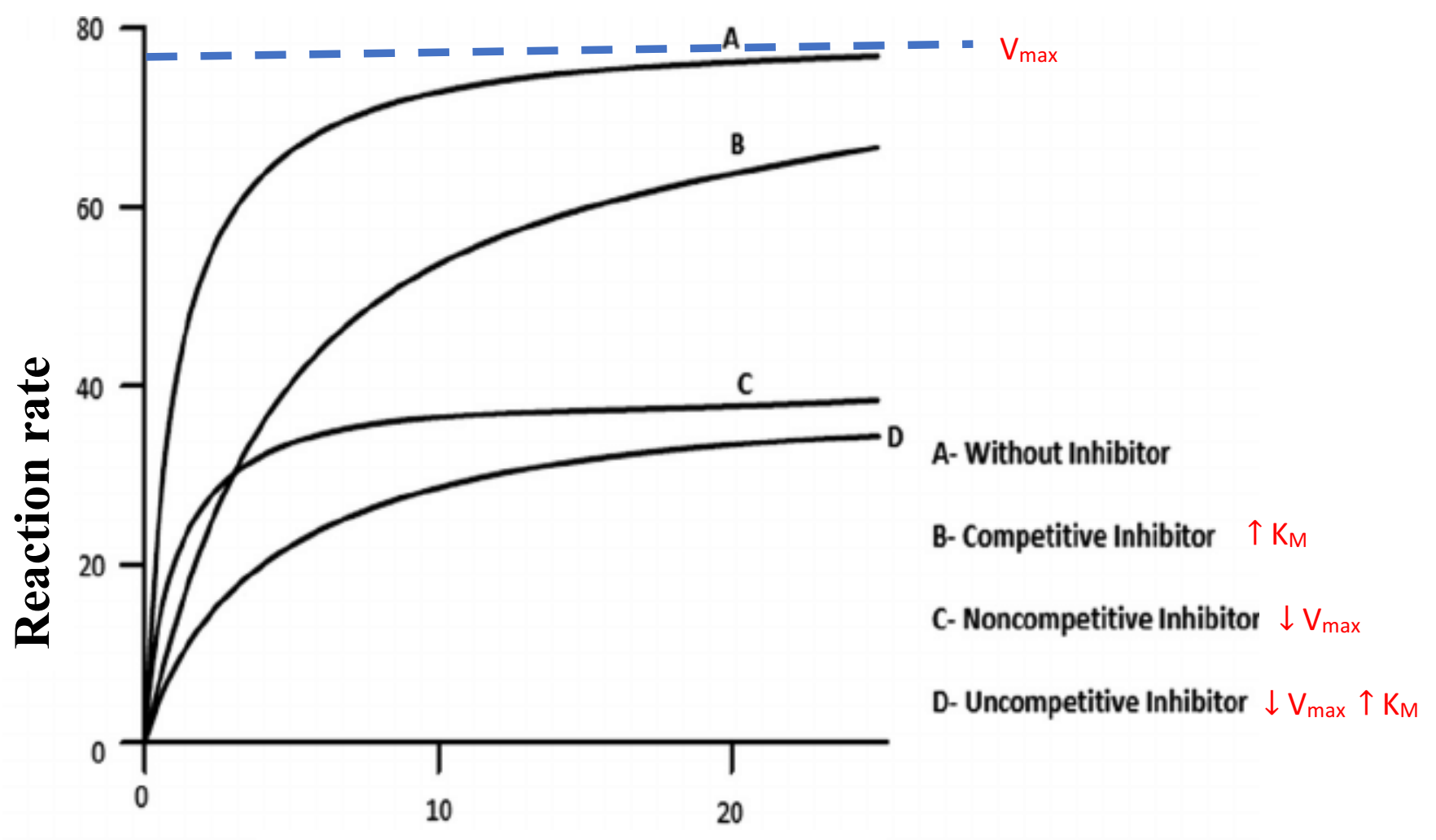

Substrate concentration

Figure 1.12: The effects of inhibition on the Michaelis-Menten curve and the constants $K_{\mathrm{M}}$ and $\mathrm{V}_{\max }$.

The Michaelis-Menten curve of the inhibited assay will provide an inhibited $K_{\mathrm{M}}$ and $\mathrm{v}_{\max }$. When compared to the uninhibited constants, the significance of the inhibition can be ascertained. An inhibition assay yielding a p-value of $<0.05$ when compared to the control indicates that the enzyme is an active catalyst for the given reaction. 


\subsection{Thesis objective}

The hypothesis heading into this thesis project is that CYP 2E1 and CYP 2C19 are responsible for the metabolism of 6:2 FTOH. This is because CYP 2C19 is responsible for the metabolism of 8:2 FTOH in the human liver and CYP 2E1's small active site would be suitable for the small, linear molecule like 6:2 FTOH. This thesis project describes the steps performed to determine the CYPs responsible for the phase I metabolism of 6:2 FTOH and calculates the MichaelisMenten constants and clearance values of 6:2 FTOH metabolism by CYP 2A6. The findings reported in this study have implications regarding the fate of 6:2 FTOH, specifically in forming bioactive metabolites, including the terminal C5-C7 PFCAs and intermediate metabolites (e.g., 6:2 FTUAL and 6:2 FTCA). The intrinsic toxicity of 6:2 FTOH can only be determined with complete knowledge of its metabolic pathway. Identifying the CYP responsible for 6:2 FTOH conversion can also help us understand whether extrahepatic toxicity might occur in cells with active levels of CYP (e.g., lung club cells) and whether there is increased risk to populations having polymorphic CYP variations. 


\section{Materials and Methods}

\section{$2.1 \quad$ Chemicals}

The solvents methanol ( $\geq 99.8 \%$ pure; HiPerSolv Chromanorm) and ethyl acetate ( $\geq 99.8 \%$ pure; ACS) was purchased from VWR. The inhibitors listed in Table 2.1 were purchased from Sigma Aldrich. The internal standard, 99\% pure 2-(Perfluoro-7-methyl octyl)ethanol (iso-9:2 FTOH), was purchased from Oakwood products inc. Mixed human liver microsomes were purchased from Sekisui Xenotech. 97\% pure 1H,1H,2H,2H-Perfluoro-1-octanol (6:2 FTOH) was purchased from Sigma Aldrich. 1H,1H,2H,2H-Perfluoro-1-decanoic acid (8:2 FTCA), 1H,1H,2H,2HPerfluoro-1-octanoic acid (6:2 FTCA) and 1H,1H,2H,2H-Perfluoro-1-octanoic acid (6:2 FTUCA) were synthesized in lab with purities $>95 \%$. Human Recombinant CYP 2 A6 is acquired from MyBioSource.

\subsection{Incubation of 6:2 FTOH with human liver microsomes}

A stock solution of $1000 \mu \mathrm{g} / \mathrm{mL}$ 6:2 FTOH was created in a solution of methanol. The addition of this solution constituted the start of the metabolic incubation. The other incubation constituents were acquired from Nabb et al ${ }^{[11]}$, with the only deviations being the final $6: 2$ FTOH concentration. The incubation was performed in $1.5 \mathrm{~mL}$ microcentrifuge tubes, with a total volume of $1 \mathrm{~mL}$. The incubation temperature of $37^{\circ} \mathrm{C}$ was kept constant by the immersion of the tube into a swirling water bath. The 6:2 FTOH was incubated in the presence of an in-lab NADPH regeneration system created in $100 \mathrm{mM} 7.4 \mathrm{pH}$ sodium phosphate buffer. The incubation system consists of $212 \mathrm{mM}$ NADP+, $0.4 \mathrm{mM}$ EDTA, 1 unit of Glucose-6-Phosphate dehydrogenase, $58 \mathrm{mM} \mathrm{MgCl}$, and $40 \mathrm{mM}$ Glucose-6-Phosphate. These are the final concentrations in the $1 \mathrm{~mL}$ incubation solution. 
The human liver microsomes were used for two distinct segments of the project. The first segment was an investigation of the effect of CYP inhibition on 6:2 FTOH metabolism, using six different selective inhibitors that target six different CYP isoforms. This will be done for two starting concentrations of 6:2 FTOH: 1000 and $10000 \mathrm{ng} / \mathrm{mL}$. The second segment will consist of creating both an uninhibited and inhibited Michaelis-Menten curve for the CYPs that show significant inhibition in the first segment.

To satisfy experiments within segment 1 , human liver microsomes were diluted in the phosphate buffer described above ( $12 \mu \mathrm{L}$ of the stock $20 \mathrm{mg} / \mathrm{mL}$ solution), yielding a final concentration of $0.24 \mathrm{mg} / \mathrm{mL}$. Human liver microsomes used to create the Michaelis-Menten curves were diluted to a final concentration of $0.20 \mathrm{mg} / \mathrm{mL}$ ( $10 \mu \mathrm{L}$ of the stock $20 \mathrm{mg} / \mathrm{mL}$ solution). The CYP inhibitor stock solutions (10 $\mu \mathrm{L})$ were each added to the buffer to yield the final concentrations described in Table 2.1. The addition of methanol greater than $2 \%$ of the total incubation volume will degrade the enzymes in the human liver microsomes. Concentrations ranged from $18-75 \mu \mathrm{M}$ and were all above the respective $\mathrm{IC}_{50}$ values. The solutions containing the NADPH regeneration system, inhibitor and active enzymes were pre-incubated for 3 minutes in the water bath at a constant temperature of $37^{\circ} \mathrm{C}$. The $6: 2 \mathrm{FTOH}$ was added $(10 \mu \mathrm{L}$ of 100 $\mu \mathrm{g} / \mathrm{mL}$ and $1000 \mu \mathrm{g} / \mathrm{mL}$ stock concentrations to yield the respective final concentrations) to initiate the reaction. 1000-fold concentration is used as if the final incubation volume of methanol exceeds $2 \%$, the integrity of the enzymes in the microsomes are affected. The incubation was performed for up to 22 hours, with samples quenched at time points 2, 1015 and 22 hours. A blank incubation is performed to be sure that the enzymatic activity is not altered over the 22-hour time frame. The blank incubation consists of the buffer, regeneration system, and the $6: 2 \mathrm{FTOH}$. 


\begin{tabular}{ll}
\hline Cytochrome P450 & Inhibitor \\
\hline CYP 2C19 & Chloramphenicol $(30 \mu \mathrm{M})$ \\
CYP 2C9 & Sulfaphenazole $(30 \mu \mathrm{M})$ \\
CYP 3A4 & Ketoconazole $(18 \mu \mathrm{M})$ \\
CYP 2E1 & Diallyl Disulfide $(68 \mu \mathrm{M})$ \\
CYP 2A6 & Tranylcypromine, HCl $(35 \mu \mathrm{M})$ \\
CYP 2D6 & \\
All CYP P450s & Quinidine $(30 \mu \mathrm{M})$ \\
& \\
\hline
\end{tabular}

Table 2.1: The names of the competitive inhibitors and the final concentration observed in the incubation well.

For the creation of the Michaelis-Menten curve, seven concentration points of the 6:2 FTOH were used to generate the linear zone $(1000,2000,4000,7000,8000,9000$ and 15000 $\mathrm{ng} / \mathrm{mL}$ ) and two concentration points will be used for the saturated plateau zone (20000 and $50000 \mathrm{ng} / \mathrm{mL})$.

All reactions were terminated after the addition of $500 \mu \mathrm{L}$ of ethyl acetate containing 4 $\mu \mathrm{M}$ of iso-9:2 FTOH internal standard and were subsequently vortexed for approximately 5 seconds. The solutions were further vortexed for $10 \mathrm{mins}$, followed by room temperature centrifugation for 10 mins at $10,000 \mathrm{rpm}$. One fraction of the organic layer $(200 \mu \mathrm{L})$ was 
removed for GC/MS analysis. Another fraction $(100 \mu \mathrm{L})$ was also extracted for LC/MS analysis, described below.

\subsection{Incubation of 6:2 FTOH with recombinant human CYP $2 A 6$}

Recombinant CYP 2A6 $(1.5 \mu \mathrm{g} / \mathrm{mL})$ was incubated with the regeneration system at $37{ }^{\circ} \mathrm{C}$ for times ranging from 17 to $51 \mathrm{hrs}$. The incubation was terminated with the addition of $500 \mu \mathrm{L}$ of ethyl acetate. It was vortexed for 10 minutes and centrifuged at 10,000 rpm for 5 minutes. The separation of the organic layer for further analysis of both the FTOH and FTUCA and FTCA were carried out as described above in section 2.2.

\section{$2.4 \quad$ Preparation of polar metabolites}

LC-MS/MS was used for the quantification of the polar metabolites formed by the human liver and recombinant CYP assays. The internal standard is 8:2 FTCA. This is chosen as an internal standard because there is no way to metabolize a higher chain FTCA (8:2) from 6:2 FTOH. The internal standard will have a similar physical characteristic to the investigated compounds. The $100 \mu \mathrm{L}$ acquired from the incubation step was spiked with $50 \mu \mathrm{L}$ of 100 $0 \mu \mathrm{g} / \mathrm{mL}$ 8:2 FTCA (prepared in methanol). The ethyl acetate solution was vacuum centrifuged down to dryness with the use of an Eppendorf vacufuge. It was then reconstituted with $200 \mu \mathrm{L}$ 50:50 Methanol/Water mixture. It was placed in a glass LC/MS vial, fitted with a polypropylene insert, and sent for analysis. 


\section{$2.5 \quad \underline{G C-M S \text { analysis }}$}

Loss of 6:2 FTOH was measured by GC-MS. The GC-MS method was acquired from Szostek et al ${ }^{[52]}$. with a few changes to the temperature program. The GC-MS used was the Agilent 6890A gas chromatograph coupled with an Agilent 5973 mass spectrometer 531 operating in EI mode. An Agilent J\&W HP-5 GC column ( $30 \mathrm{~m} \times 0.25 \mathrm{~mm}$ x $0.25 \mu \mathrm{m})$ was used for all analysis. The flow rate was set to $1.0 \mathrm{~mL} / \mathrm{min}$ for the analysis of $6: 2 \mathrm{FTOH}$ from the recombinant CYP assay, while a flow rate of $0.8 \mathrm{~mL} / \mathrm{min}$ was used for the human liver microsomes assay. The temperature program stayed constant across all assay analysis: initial temp $\left(60^{\circ} \mathrm{C}\right)$, held for $2 \mathrm{~min}$, ramped to $120^{\circ} \mathrm{C}\left(10^{\circ} \mathrm{C} / \mathrm{min}\right)$, held for $1 \mathrm{~min}$, ramped to $250^{\circ} \mathrm{C}$ $\left(50{ }^{\circ} \mathrm{C} / \mathrm{min}\right)$, held for $3 \mathrm{~min}$. Each sample was injected $(2 \mu \mathrm{L})$ with a $10: 1$ spitless mode, and 250 ${ }^{\circ} \mathrm{C}$ inlet temperature. Single ion monitoring (SIM) of 95, 131 and $364 \mathrm{~m} / \mathrm{z}$ were used for identification and quantification of the 6:2 FTOH and iso-9:2 FTOH. All rates calculated from these measurements were absolute, to remove the negative sign of 6:2 FTOH loss.

\section{6 $\quad \underline{L C-M S / M S \text { analysis }}$}

The LC-MS/MS method used is provided by Zhang et al ${ }^{[89]}$ with a few modifications. The instrument used was a Waters ultra-performance liquid chromatography (UPLC) system with a XevoTQS tandem mass spectrometer (MS/MS) with electrospray ionization (ESI). The organic mobile phase (B) was composed of $100 \%$ methanol with $2 \mathrm{mM}$ ammonium acetate and $5 \mathrm{mM} \mathrm{1-}$ methyl piperidine. The aqueous mobile phase (A) was composed of $70 \%$ water and $30 \%$ methanol with $2 \mathrm{mM}$ ammonium acetate and $5 \mathrm{mM}$ 1-methyl piperidine. The flow rate was constant at $0.3 \mathrm{~mL} / \mathrm{min}$. The inlet method starts with a 2.5 -minute hold of $60: 40 \mathrm{~A}: \mathrm{B}$. The mixture changed to $25: 75$ over 3.5 minutes. The $25: 75$ mixture is held for 1 minute, reverting to 
initial conditions (60:40 A: B) over the coursoverIt is then held for 1 minute. The MS dwell time was set at $0.020 \mathrm{~s}$. The cone voltage was $14 \mathrm{~V}$. The collision energy was $16 \mathrm{~V}$ for the $6: 2$ FTUCA and $22 \mathrm{~V}$ for both the 6:2 and 8:2 FTCA. The MRM for 6:2 FTUCA was 356.7 $>293.0$ $m / z$. The MRM for $6: 2$ FTCA is $377.0>293.0 \mathrm{~m} / \mathrm{z}$. The MRM for $8: 2$ FTCA is $477.0>393.0$ $m / z$

\subsection{Statistics}

Triplicate analysis was performed for all time points in rate calculations in the preliminary inhibition assays. For the creation of the Michaelis-Menten curve, each concentration point was derived from a 6:2 FTOH concentration vs time plot. Triplicates are performed for each data point in the concentration vs time plots. Both the One tail t-test and oneway ANOVA analysis (Bonferroni correction post hoc) were used to characterize significant differences amongst two or more groups, respectively. Each statistical analysis was performed on Excel 2020.

\subsection{Quality control}

Spike and recovery data is provided in Table S1 in the supplementary section. Spike and recovery experiments were performed for the extraction of 6:2 FTOH from the inhibitor incubation assays, at two 6:2 FTOH concentrations (1000 and $10000 \mathrm{ng} / \mathrm{mL}$ ). The time point used for the spike and recovery was 2 hours. For the creation of the Michaelis- Menten curve, spike and recovery experiments were performed for all the 6:2 FTOH concentrations tested. It was performed without the $10 \mu \mathrm{L}$ inhibitor addition. The time point used was 2 hours.

The Limit of Detection (LOD) and Limit of Quantification (LOQ) of the GC-MS used was acquired from the triplicate-made standard curve. The LOD and LOQ are set as:

$$
\mathrm{LOD}=3 \frac{\sigma}{m}
$$


Where $\sigma$ represents the average standard deviation of all three curves and $\mathrm{m}$ represents the average slope of the standard curve. The LOQ is determined by the equation.

$$
\mathrm{LOQ}=10 \frac{\sigma}{m}
$$

The LOD and LOQ are present in table 2.1.

LOD LOQ

\begin{tabular}{l|ll} 
6:2 FTOH & $15.5 \mathrm{ng} / \mathrm{mL}$ & $51.6 \mathrm{ng} / \mathrm{mL}$ \\
6:2 FTUCA & $6.8 \mathrm{pg} / \mathrm{mL}$ & $22.6 \mathrm{pg} / \mathrm{mL}$
\end{tabular}

Table 2.2: The limit of detection and limit of quantitation for the 6:2 FTOH and 6:2 FTUCA. 


\section{6:2 FTOH metabolizes by CYP $3 \mathrm{~A} 4$ and $2 \mathrm{~A} 6$ in human liver microsomes}

8:2 FTOH is metabolized by CYP 2E1 and CYP 2C19 in rats and humans respectively ${ }^{13,14}$. This demonstrates that there is a wide variety of CYPs that could be responsible for the metabolism of the shorter chain length analogue, 6:2 FTOH. As the 6:2 FTOH may not be metabolized by either CYP2E1 or 2C19, the study expanded the list of investigated enzymes to incorporate several more CYPs of toxicological significance. The CYPs investigated in this project were selected due to their prevalence in the liver as well as their prevalence in known toxicant activation metabolism ${ }^{[87]}$. The remaining CYPs investigated are shown in Table 1, and include CYP 2A6, CYP 3A4, CYP 2C9, and CYP 2D6 . To do this, we incubated human liver microsomes containing 6:2 FTOH both in the presence and absence of selective CYP inhibitors and compared 6:2 FTOH levels to uninhibited control levels. Inhibitor(s) will cause the depression of the 6:2 FTOH metabolic rate and provide a list of CYPs responsible for 6:2 FTOH metabolism. Two concentrations of 6:2 FTOH were chosen for this stage: $1000 \mathrm{ng} / \mathrm{mL}$ and $10000 \mathrm{ng} / \mathrm{mL}$, which signified low and high concentrations, respectively. The assay was first optimized to determine the times at which 6:2 FTOH levels were significantly reduced from the assay start ( $\mathrm{T}=0$ hours). The 6:2 FTOH levels were measured approximately every 5 hours, up to 22 hours. A previous study showed significant depletion of 6:2 FTOH over 2 to 48 hours ${ }^{13}$. The final time for the assay was 22 hours, and it took about 2 hours for 6:2 FTOH levels to be significantly different from the LOQ. This provided four-time points from which the reaction rate could be calculated. All inhibitors used were competitive towards a given CYP. The inhibition trials provided a metabolic rate that was compared to the control (uninhibited rate) 
with the use of a one-way ANOVA test. Here, an ANOVA test was performed instead of a t-test because the statistical difference of all 8 inhibition assays was required. This cannot be performed with a t-test. The null hypothesis of the ANOVA test seeks to prove that there is no inhibition of the reaction rate in the presence of a specific inhibitor.

Unlike results from previous studies, which showed that 8:2 FTOH metabolism was controlled by CYP2E1 and 2C19 [55][91], inhibition of CYP2E1 and CYP2C19 did not significantly reduce the 6:2 FTOH metabolic rate at both tested concentrations. As seen in Figure 3.1 and 3.2, the use of chloramphenicol (CYP 2C19) and allyl disulfide (CYP 2E1) did not result in a significant reduction in the loss of 6:2 FTOH. As seen in Table 3.1, a one-way ANOVA test of CYPs 2E1 and 2C19, provided a p-value of 0.12 and 0.77 , which exceeds an $\alpha$ of 0.05 . The null hypothesis cannot be rejected and thus the significant difference between the metabolic rates of those inhibited runs and the control cannot be ascertained. However, comparing the control and all inhibitor treatments, denoted as the "all trials" ANOVA, provided a p-value of 5.90E-07. This means the null hypothesis is rejected and the assay consists of at least one treatment that is significantly different from another. When factoring in the use of an inhibitor that inhibits all CYPs (1-Aminobenzotriazole) the p-values are 0.004 and 0.0006 for the $1000 \mathrm{ng} / \mathrm{mL}$ and 10000 $\mathrm{ng} / \mathrm{mL}$ assays respectively. These $\mathrm{p}$-values are lower than 0.05 and the null hypothesis must be rejected. The rejection of all CYPs inhibition assay demonstrates that the metabolism of 6:2 FTOH in the human liver is significantly catalysed by a CYP. For both the lower and higher concentration trials, the CYP $2 \mathrm{~A} 6$ and CYP $3 \mathrm{~A} 4$ results provided a p-value low enough to reject the null hypothesis. The all-CYPs inhibitor ANOVA values coupled with the individual CYP assay leads to the conclusion that CYP 2A6 and CYP 3A4 are the two CYPs responsible for the metabolism of 6:2 FTOH to 6:2 FTAL 


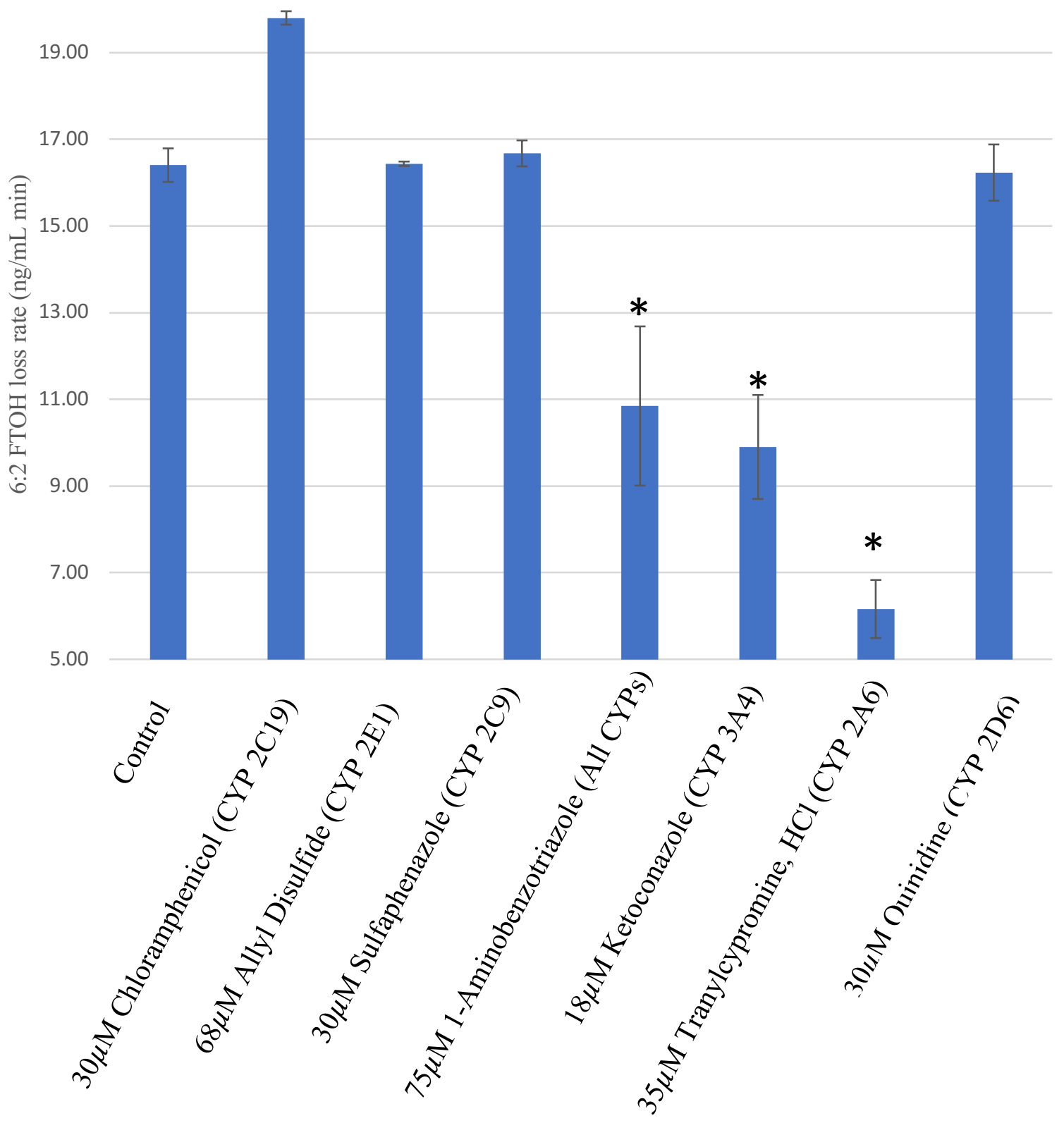

Figure 3.1: Metabolic rates of 6:2 FTOH conversion in the presence of competitive inhibitors. The starting concentration of 6:2 FTOH is $1000 \mathrm{ng} / \mathrm{mL}$. Standard deviations were taken from $\mathrm{n}=3$ trials. "*" symbolizes a $\mathrm{p}$-value $<0.05$ in a one-way t-test. 


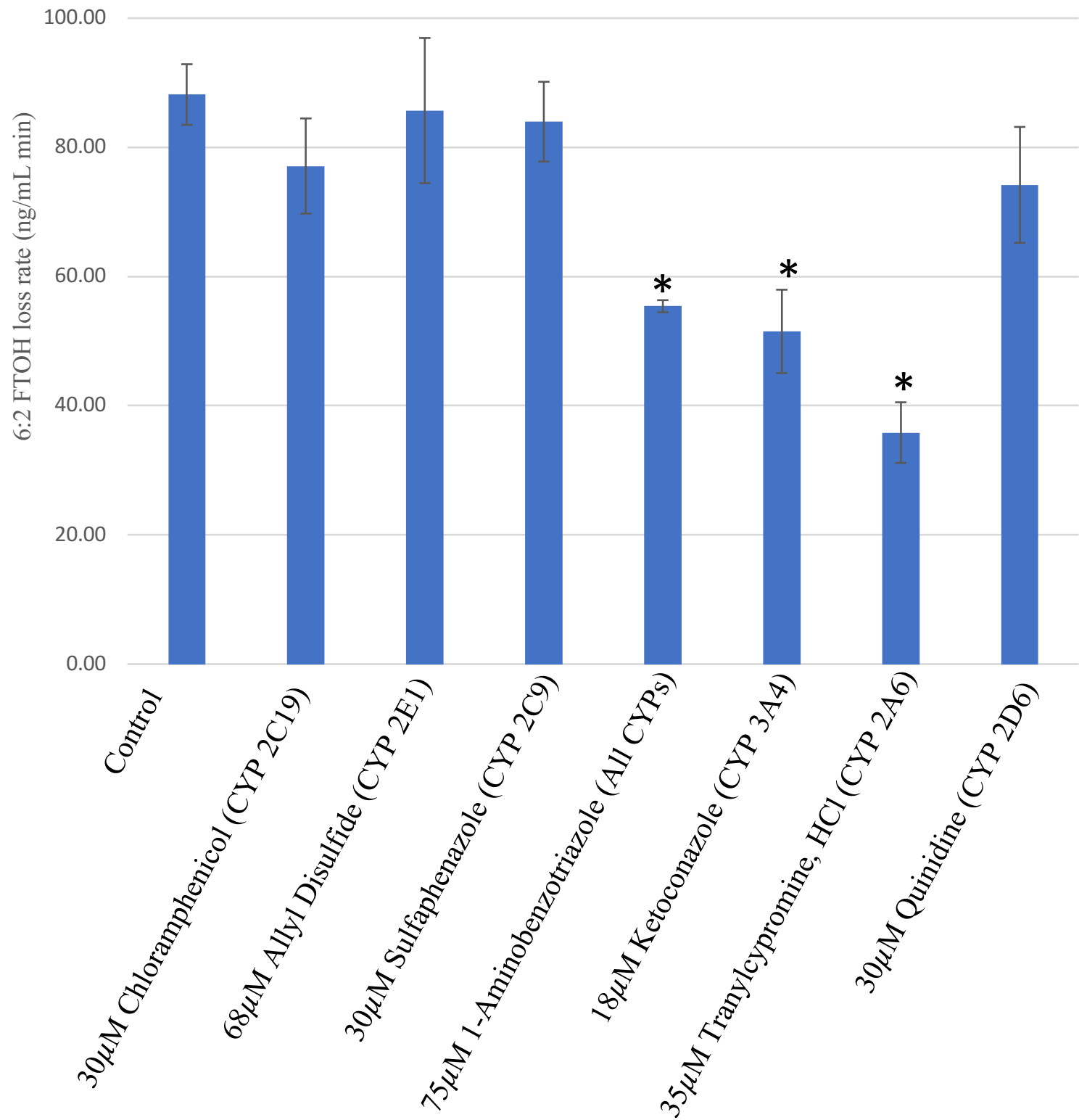

Figure 3.2: Metabolic rates of 6:2 FTOH conversion in the presence of competitive inhibitors. The starting concentration of 6:2 FTOH is $10000 \mathrm{ng} / \mathrm{mL}$. Standard deviations were derived from $\mathrm{n}=3$ trials. "**" symbolizes a $\mathrm{p}$-value $<0.05$ in a one-way t-test. 
CYP 2C19 (30 $\mu \mathrm{M})$

CYP 2E1 (68 $\mu \mathrm{M})$

CYP 2C9 $(30 \mu \mathrm{M})$

ALL CYPS $(75 \mu \mathrm{M})$

CYP 3A4 (18 $\mu \mathrm{M})$

CYP 2A6 $(50 \mu \mathrm{M})$

CYP 2D6 $(30 \mu \mathrm{M})$

All trials

Table 3.1: The p-values corresponding with a one-way ANOVA test between the uninhibited and inhibited 6:2 FTOH metabolic rates at 1,000 and 10,000 ng/mL. "All trials" signify the ANOVA test comparing the uninhibited with all the inhibited trials. 
Results from this experiment showed that CYP 2A6 and CYP 3A4 are responsible for 6:2 FTOH transformation in human liver microsomes. The Michaelis- Menten constant $\left(K_{\mathrm{M}}\right)$ and $\mathrm{V}_{\max }$ will be used to determine $6: 2 \mathrm{FTOH}$ clearance rates and a one-tail t-test performed to determine the significance of the difference. By calculating the $K_{\mathrm{M}}$ and $\mathrm{V}_{\max }$, we can also verify the type of inhibition produced. Further analysis will have to be conducted in future for CYP $3 \mathrm{~A} 4$ to determine which $\mathrm{CYP}(\mathrm{s})$ is primarily responsible (CYP3A4 vs. 2A6) for a significant portion of 6:2 FTOH metabolism. 


\section{Uninhibited and inhibited Michaelis Menten curves}

CYP 2A6 was chosen for further study due to the ANOVA p-values shown in Table 3.1. It was one of the two CYPs shown to have significant inhibition for 6:2 FTOH concentrations. It expressed the most inhibition, therefore it was chosen as the primary CYP of interest. For the creation of the uninhibited Michaelis-Menten (control), the method used is described in section 2.3. The lowest concentration used for the formation of the curve was $1000 \mathrm{ng} / \mathrm{mL}$, which was the low concentration used to determine which CYP was involved in the 6:2 FTOH transformation, described in the above section. There are six different concentrations within the linear zone, ranging from 1000 - 9000 and ending around 15000 and $20000 \mathrm{ng} / \mathrm{mL}$. The plateau zone is fitted with one concentration: $50,000 \mathrm{ng} / \mathrm{mL}$. These 8 concentrations are enough to model the Michaelis-Menten curve and provide a $K_{\mathrm{M}}$ and $\mathrm{V}_{\mathrm{Max}}$ with high confidence ${ }^{[88]}$. At each concentration, the $6: 2 \mathrm{FTOH}$ loss rate was calculated in triplicate over time (0-22 hours). The method for the generation of the Michaelis-Menten curve is described in Section 2.3. Triplicate analysis was used to calculate the standard deviation, which will be shown as the y-error bars in the Michaelis-Menten graph (Fig 4.1). 


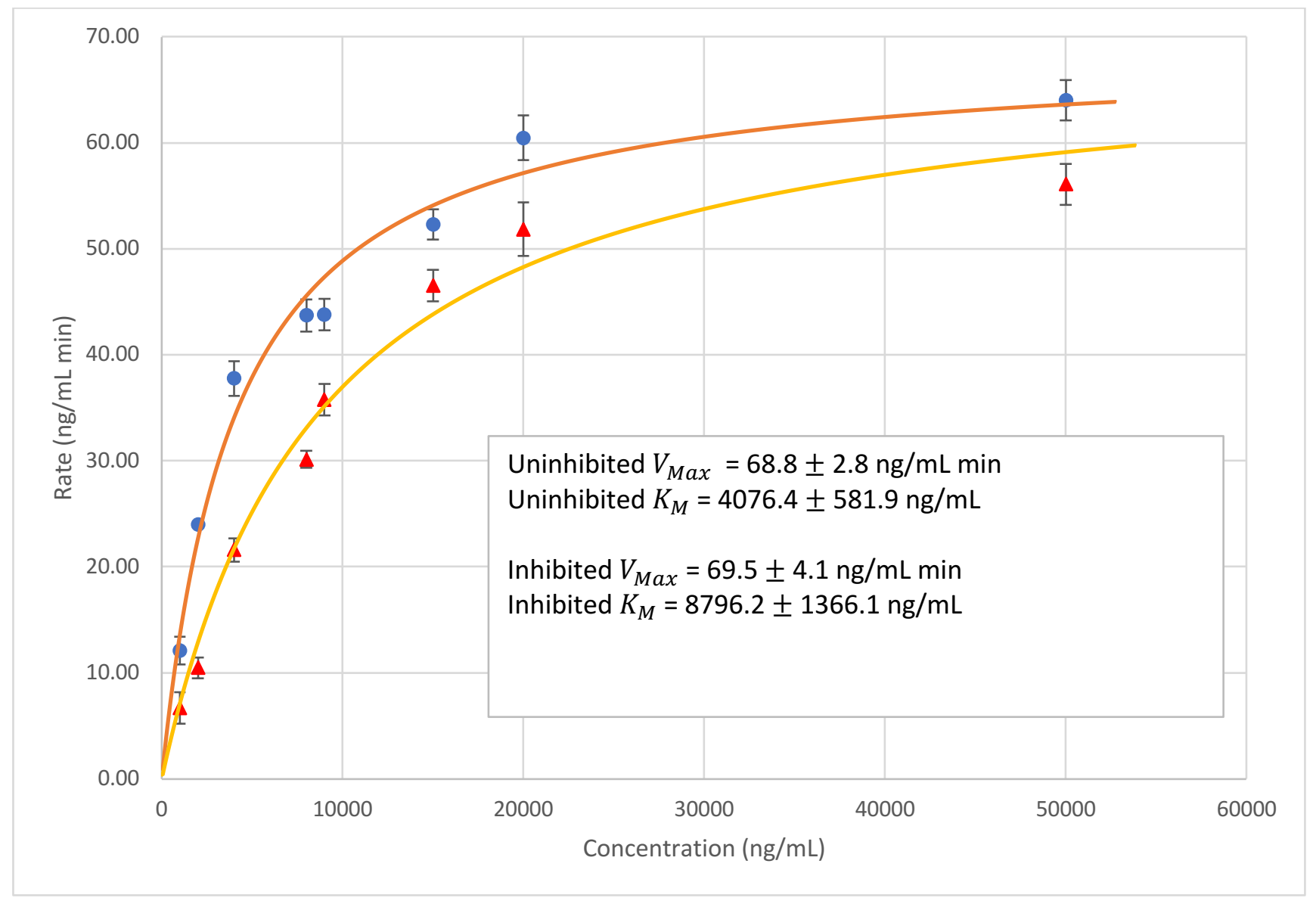

Figure 4.1: Michaelis-Menten plot of 6:2 FTOH biotransformation in human liver microsomes $(0.20 \mathrm{mg}) .6: 2 \mathrm{FTOH}$ is incubated in the presence of an NADPH regeneration system at $37{ }^{0} \mathrm{C}$ for time points ranging from 0 to 22 hours. The Michaelis-Menten constant for the biotransformation in the absence of CYP 2A6 inhibition (blue circles) is $4076.4 \pm 581.9 \mathrm{ng} / \mathrm{mL}$, and the maximum rate of $68.8 \pm 2.8 \mathrm{ng} / \mathrm{mL} / \mathrm{min}$. The Michaelis-Menten constant in the presence of CYP 2A6 inhibitor (red triangles) is $8796.2 \pm 1366.1 \mathrm{ng} / \mathrm{mL}$, and the maximum rate of $69.5 \pm$ $4.1 \mathrm{ng} / \mathrm{mL} / \mathrm{min}$. The constants were obtained through nonlinear regression using MatLab. Error bars indicate standard deviation acquired from $n=3$ trials. 
The data and their respective errors were then fitted to the Michaelis-Menten equation using MatLab. The Michaelis-Menten graph provided a $K_{\mathrm{M}}$ of $4076.4 \pm 581.9 \mathrm{ng} / \mathrm{mL}$ and a $\mathrm{V}_{\text {Max }}$ of $68.8 \pm 2.8 \mathrm{ng} / \mathrm{mL} / \mathrm{min}$. The inhibition curve is performed with the same method, in the presence of $35 \mu M$ tranylcypromine, $\mathrm{HCl}$. The inhibited Michaelis-Menten curve provided a $\mathrm{K}_{\mathrm{M}}$ of 8796.2 $\pm 1366.1 \mathrm{ng} / \mathrm{mL}$ and a $\mathrm{V}_{\mathrm{Max}}$ of $69.5 \pm 4.1 \mathrm{ng} / \mathrm{mL} \min$. The $\mathrm{K}_{\mathrm{M}}$ doubles in the presence of the inhibitor. This means that the affinity of the substrate to CYP 2A6's binding site is significantly reduced in the presence of the inhibitor. When the $\mathrm{V}_{\text {Max }}$ was adjusted to protein levels $(0.20$ $\mathrm{mg} / \mathrm{mL}$ of liver microsome), the specific $\mathrm{V}_{\mathrm{Max}}$ is $344 \pm 28 \mathrm{ng} / \mathrm{min} \mathrm{mg}$ for the uninhibited incubation and $347 \pm 41 \mathrm{ng} / \mathrm{min} \mathrm{mg}$ for the inhibited incubation.

The similar $\mathrm{V}_{\mathrm{Max}}$ of the uninhibited and inhibited curve, coupled with their different $K_{\mathrm{M}}$ value, illustrates a competitive inhibition of CYP 2 A6 by Tranylcypromine, $\mathrm{HCl}$. The 95\% confidence interval for each constant is generated through MatLab and are displayed in Table 4.1.

\begin{tabular}{l|cc}
\multicolumn{2}{l}{ Value } & 95\% Interval \\
\hline Uninhibited $\boldsymbol{V}_{\boldsymbol{M a x}}$ & $68.8 \pm 2.8 \mathrm{ng} / \mathrm{mL} \mathrm{min}$ & $65.6-72.2 \mathrm{ng} / \mathrm{mL} \mathrm{min}$ \\
\hline Inhibited $\boldsymbol{V}_{\boldsymbol{M a x}}$ & $69.5 \pm 4.1 \mathrm{ng} / \mathrm{mL} \mathrm{min}$ & $64.9-74.1 \mathrm{ng} / \mathrm{mL} \mathrm{min}$ \\
Uninhibited $\boldsymbol{K}_{\boldsymbol{M}}$ & $4076.4 \pm 581.9 \mathrm{ng} / \mathrm{mL}$ & $3420-4730 \mathrm{ng} / \mathrm{mL}$ \\
Inhibited $\boldsymbol{K}_{\boldsymbol{M}}$ & $8796.2 \pm 1366.1 \mathrm{ng} / \mathrm{mL}$ & $7250-10300 \mathrm{ng} / \mathrm{mL}$ \\
\end{tabular}

Table 4.1: $\mathrm{V}_{\mathrm{Max}}$ and $K_{\mathrm{M}}$ and their respective $95 \%$ confidence interval for both the uninhibited and inhibited Michaelis-Menten curve.

The $\mathrm{V}_{\mathrm{Max}}$ values overlap while there is no overlap between the $K_{\mathrm{M}}$ values. This confirms the competitive inhibition of CYP 2A6 and its effect on the metabolism of 6:2 FTOH in liver microsomes. 
The calculation of the $K_{\mathrm{M}}$ and $\mathrm{V}_{\mathrm{Max}}$ allows for the intrinsic clearance rate, $\mathrm{CL}_{\text {int }}$, to be determined. It is one of the constants used to determine the enzymes responsible for the elimination of a compound from a given organ. This is an in vitro variable that does not account for metabolism and elimination outside of the organ of choice (in this case, the liver). For a more precise source of 6:2 FTOH metabolism in the human body, an in vivo study must be performed, although many recent pharmacokinetic computational models rely on in vitro clearance rates to predict the overall fate of a xenobiotic chemical. In vitro to in vivo extrapolation (IVIVE) is an extrapolation of in vitro experimental data to in vivo effects. The clearance rate is a vital in-vivo parameter for pharmacokinetics. It determines the rate at which a given drug is eliminated from the body from the moment of absorption. Intrinsic clearance seeks to model the clearance rate in an in vitro assay. $\mathrm{CL}_{\text {int }}$ measures the hepatic clearance of a given compound without the parameters of blood flow (transportation) and binding affinity ${ }^{[89]}$. $\mathrm{CL}_{\text {int }}$ is calculated by dividing the specific $\mathrm{V}_{\mathrm{Max}}$ by the $K_{\mathrm{M}}$. The $\mathrm{CL}_{\text {int }}$ for the uninhibited and inhibited trials are $0.084 \pm 0.015$ $\mathrm{mL} / \mathrm{min} \mathrm{mg}$ and $0.040 \pm 0.004 \mathrm{~mL} / \mathrm{min} \mathrm{mg}$. A one-tail T-test is performed and the $\mathrm{CL}_{\text {int }}$ for the inhibited and uninhibited trials are statistically significant. From the T-test result, there is reasonable confidence in concluding that CYP 2A6 plays a significant role in the metabolism and elimination of 6:2 FTOH in the human liver. 


\section{6:2 FTOH metabolism in human recombinant CYP 2A6}

Inhibition of enzymes involves the attachment of a non-substrate into an active site of either the enzyme or the enzyme-substrate complex. It is difficult to find a compound that will only fit into a specific active site. Some compounds are strong inhibitors for a certain CYP but also exhibit weak inhibition of a different CYP. For example, tranylcypromine, $\mathrm{HCl}$ strongly inhibits CYP 2 A6 but shows weak inhibition to CYP 3A4. For this reason, a recombinant human CYP assay must be performed to conclude that the inhibition produced in the liver microsome assay is solely from the CYP in question. The metabolic rate of 6:2 FTOH in human recombinant CYP 2A6 was determined with an enzyme concentration of $1.5 \mu \mathrm{g} / \mathrm{mL}$. Two 6:2 FTOH concentrations were used: 1000 and $10000 \mathrm{ng} / \mathrm{mL}$. Control experiments were performed, where 6:2 FTOH was incubated in CYP 2A6 without an inhibitor, as well as those performed with an inhibitor. The inhibitor concentrations tested for the $10000 \mathrm{ng} / \mathrm{mL} 6: 2 \mathrm{FTOH}$ assays were $35 \mu \mathrm{M}$ and $17.5 \mu \mathrm{M}$, while the concentration for the $1000 \mathrm{ng} / \mathrm{mL} 6: 2 \mathrm{FTOH}$ assay was $17 \mu \mathrm{M}$. This is above the $\mathrm{IC}_{50}$ value of $0.42 \mu \mathrm{M}$ for tranylcypromine $\mathrm{HCl}$. An additional inhibitor concentration was used for the higher $6: 2 \mathrm{FTOH}$ starting concentration in the situation that $17.5 \mu \mathrm{M}$ would not be enough to cause significant depression of the metabolic rate. The 6:2 FTOH loss was measured for the time points 17, 20, 25, 31 hours. As shown in Figure 5.1, the 6:2 FTOH (10 $000 \mathrm{ng} / \mathrm{mL})$ metabolic rates for the inhibited assays were 14- and 84-times less than the uninhibited control, with rate values of $0.031 \pm 0.007 \mathrm{ng} / \mathrm{mL} \cdot \min , 0.005 \pm 0.002 \mathrm{ng} / \mathrm{mL} \cdot \mathrm{min}$, and $0.42 \pm 0.08 \mathrm{ng} / \mathrm{mL} \cdot \mathrm{min}$ for the $17.5 \mu \mathrm{M}$ inhibition, $35 \mu \mathrm{M}$ inhibition, and uninhibited control, respectively. Figure 5.2 shows a similar trend, where the $6: 2 \mathrm{FTOH}(1000 \mathrm{ng} / \mathrm{mL})$ inhibited metabolic rates were about 4-times less than the uninhibited control, with rate values of $0.04 \pm 0.009 \mathrm{ng} / \mathrm{mL} \cdot \mathrm{min}$ and $0.15 \pm 0.005$ 
$\mathrm{ng} / \mathrm{mL} \cdot \min$ for the $35 \mu \mathrm{M}$ inhibition and uninhibited control, respectively. For both 6:2 FTOH concentrations, there is a statistical difference between the control and the inhibited run at $35 \mu \mathrm{M}$ Tranylcypromine, $\mathrm{HCl}$. For the assay containing the high 6:2 FTOH concentration (10000 $\mathrm{ng} / \mathrm{mL}$ ) assay, there is a statistical difference between the $17.5 \mu \mathrm{M}$ Tranylcypromine, $\mathrm{HCl}$ and the uninhibited control. However, there is no statistical difference between the two inhibitor concentrations (17.5 $\mu \mathrm{M}$ and $35 \mu \mathrm{M})$. Together, these results confirm the role of CYP 2A6 in the metabolism of 6:2 FTOH in humans. In addition, a blank run that consisted of an incubation assay with no recombinant enzyme, was performed along with each time point to determine the background loss (e.g., via abiotic oxidation, adsorption, etc.) of 6:2 FTOH. The blank results were statistically different to the uninhibited control assay, but there was no statistical difference compared to the two inhibitor assays. This difference between the uninhibited control and the blank run provides further confirmation of the role of CYP $2 \mathrm{~A} 6$ in the metabolism of 6:2 FTOH in humans. 


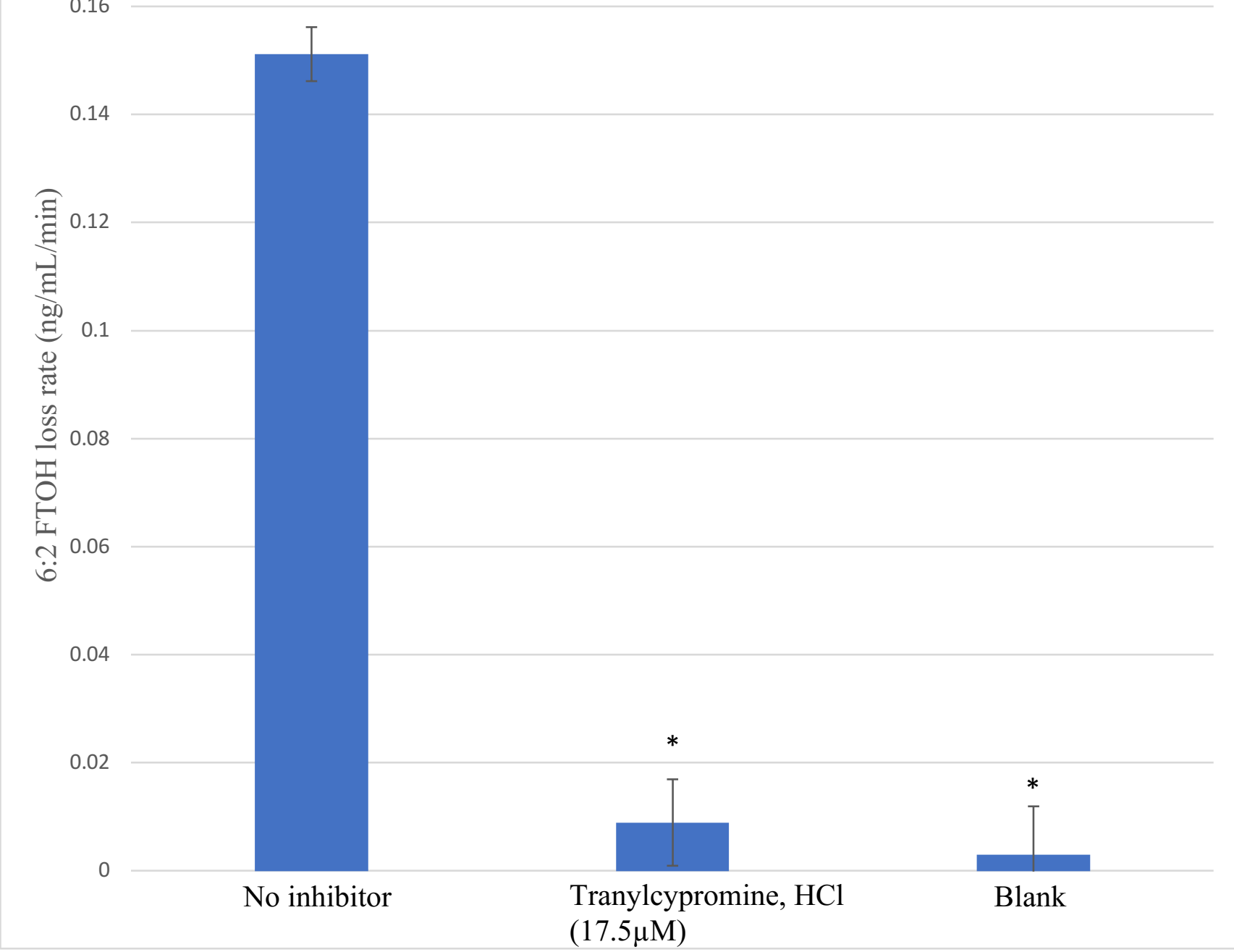

Figure 5.1: Metabolic rates of inhibited and uninhibited 6:2 FTOH conversion in the presence of human recombinant CYP 2A6. The starting concentration of $6: 2 \mathrm{FTOH}$ is $1000 \mathrm{ng} / \mathrm{mL}$. The concentration of CYP $2 \mathrm{~A} 6$ is $0.3 \mathrm{ng} / \mathrm{mL}$. Standard deviations were derived from $\mathrm{n}=3$ trials. "*” symbolizes a p-value $<0.05$ in a one-way t-test when compared to the control. 


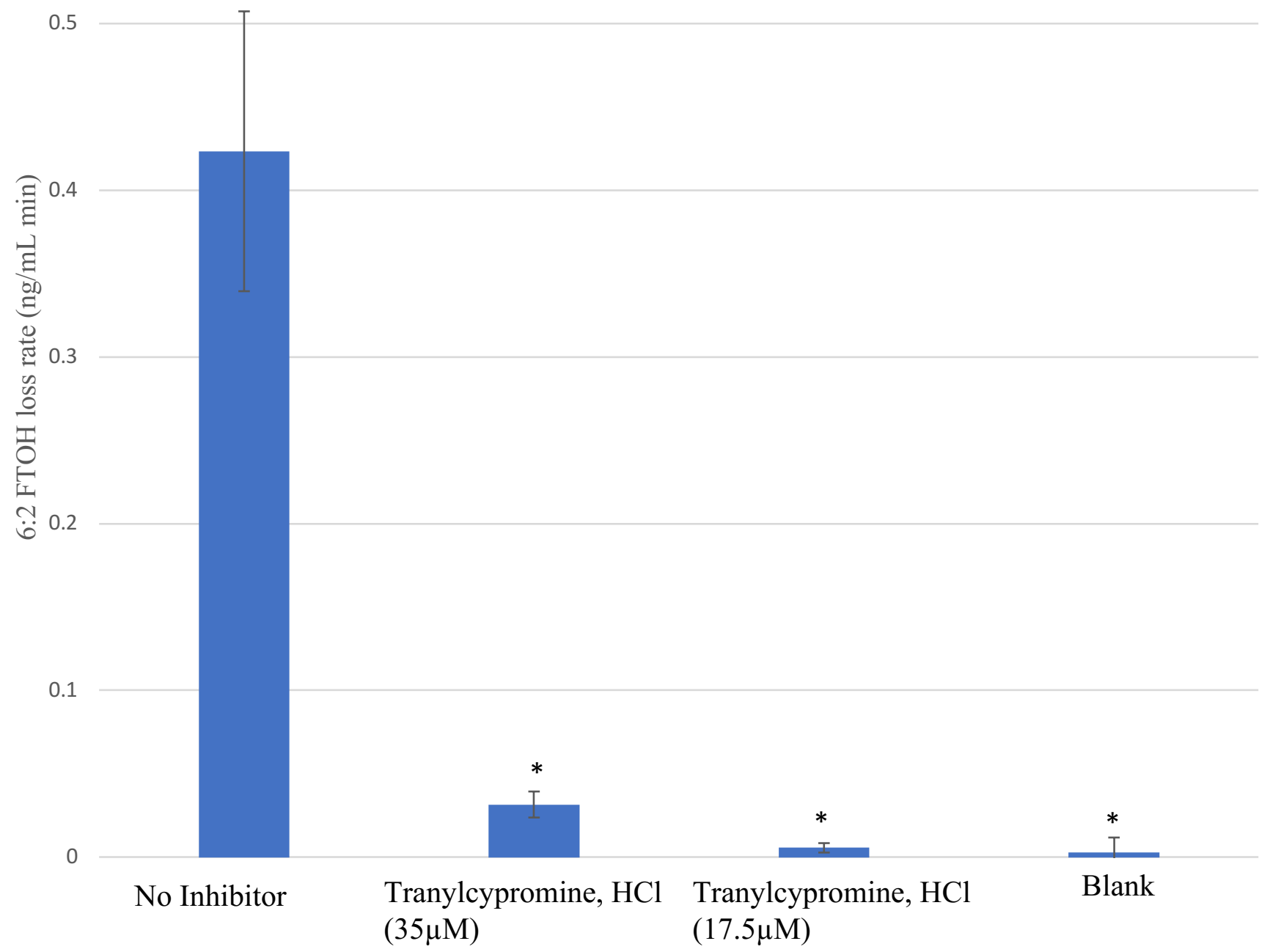

Figure 5.2: Metabolic rates of inhibited and uninhibited 6:2 FTOH conversion in the presence of Human recombinant CYP 2A6. The starting concentration of $6: 2 \mathrm{FTOH}$ is $10000 \mathrm{ng} / \mathrm{mL}$. The starting concentration of CYP $2 \mathrm{~A} 6$ is $0.3 \mathrm{ng} / \mathrm{mL}$. Standard deviations were derived from $\mathrm{n}=3$ trials. "*” symbolizes a p-value $<0.05$ in a one-way t-test when compared to the control. 


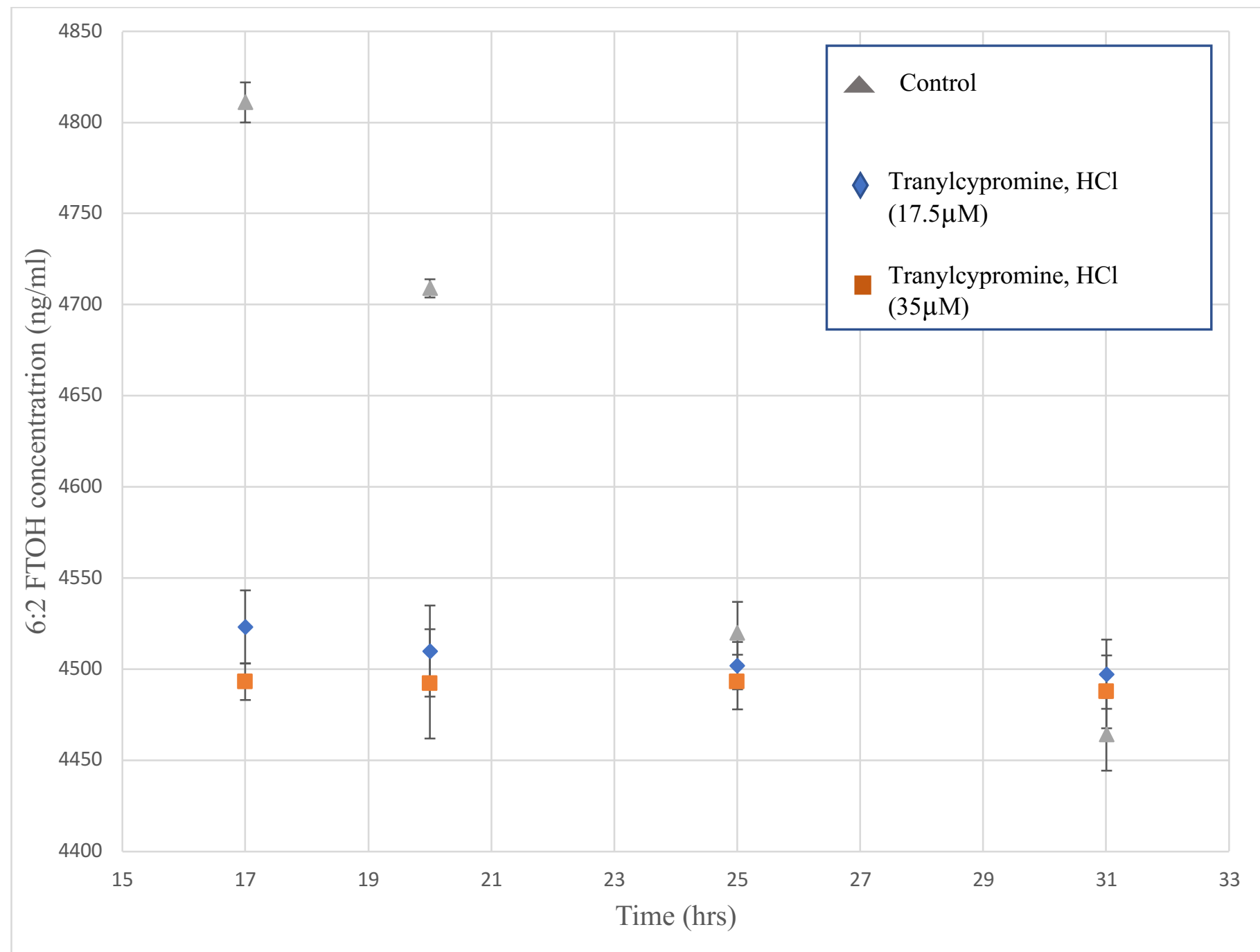

Figure 5.3: Metabolic rates of inhibited and uninhibited 6:2 FTOH conversion in the presence of human recombinant CYP 2A6. The starting concentration of 6:2 FTOH is $10000 \mathrm{ng} / \mathrm{mL}$. The concentration of CYP 2 A6 is $0.3 \mathrm{ng} / \mathrm{mL}$. Standard deviations were derived from $\mathrm{n}=3$ trials. 


\section{Discussion}

\subsection{6:2 FTOH metabolism by CYP $2 A 6$}

CYP 2E1 and CYP 2C19 are responsible for the metabolism of 8:2 FTOH in rat hepatocytes and human liver microsomes, respectively ${ }^{[55][90]}$. CYP 2E1 is primarily localized in the liver [82][57]. While it does not constitute a large percentage of P450s in the liver, it can bioactive several environmental toxicants and carcinogens like nitrosamines and azo compounds ${ }^{[83]}$. We had originally hypothesized CYP C19 to be the predominant CYP for 6:2 FTOH conversion. Given that 8:2 FTOH is catalysed by CYP $2 \mathrm{C} 19$, the starting hypothesis was to assume that CYP 2C19 would have significant involvement in the metabolism of smaller FTOHs.

CYP $2 \mathrm{~A} 6$ is another prevalent hepatic enzyme. It is also expressed in the nasal mucosa, although at low levels ${ }^{[95]}$. When compared to other CYPs like CYP 3A4 and 2D6, it contributes only a small percentage towards the metabolism of pharmaceutical substrates ${ }^{\left[{ }^{[6]}\right.}$. It was initially considered less important in the metabolism of xenobiotics compared to CYP 3A4 and 2E1, due to its restrictive active site which reduces the number of substrates ${ }^{[96]}$. CYP 2 A6 is the only CYP involved in the 7-hydroxylation of coumarin ${ }^{[96]}$. Coumarin is a bulky molecule with two fused benzene rings. Other well-known CYP 2A6 substrates, like nicotine and cotinine, are composed of an aromatic ring. Examples of CYP 2A6 substrates are shown in figure 6.1. 

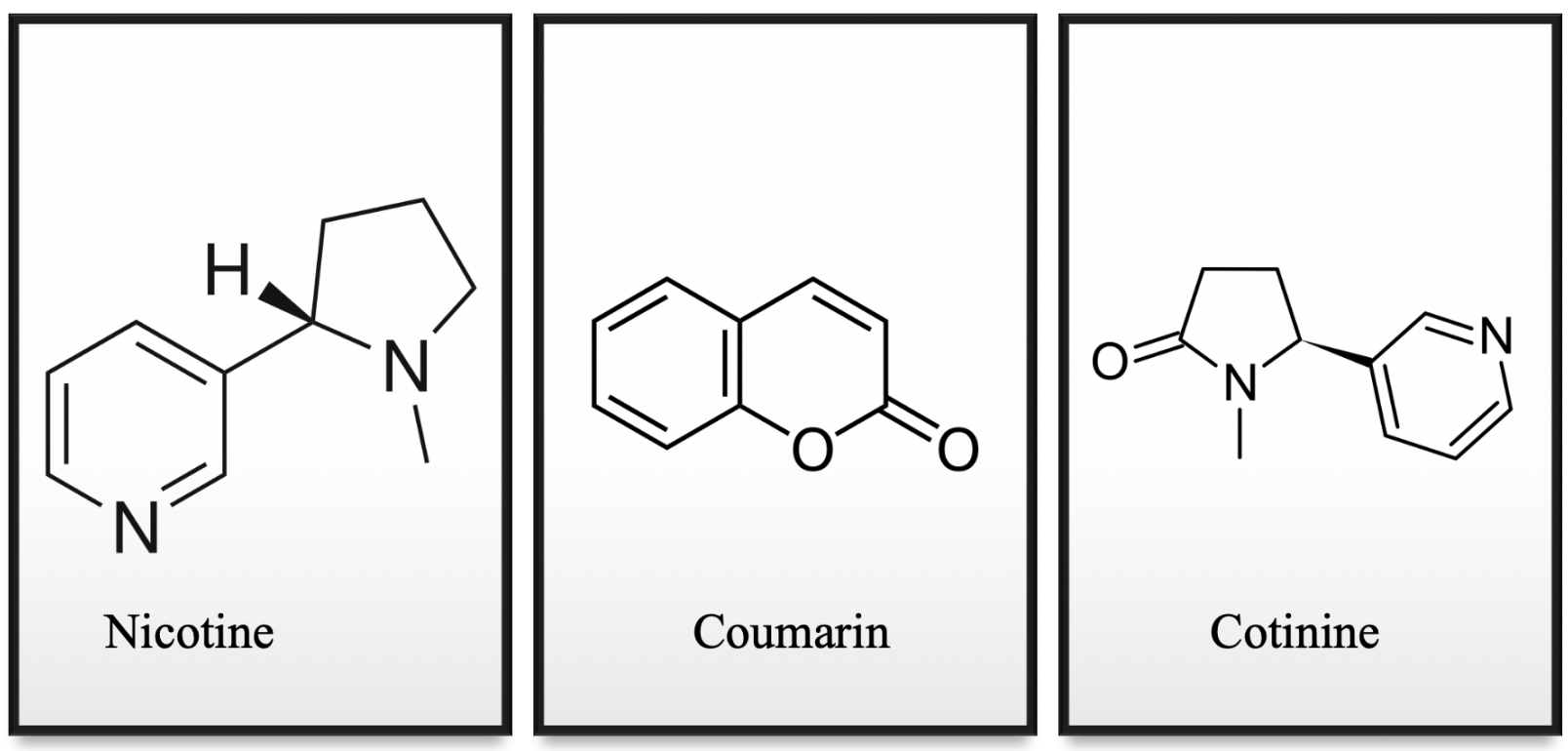

Figure 6.1: CYP 2A6 substrates: Nicotine, Coumarin and Cotinine

CYP 2A6 substrates are known to be slightly polar with low to medium molecular weights. The 6:2 FTOH does not have an aromatic ring. Its molecular weight of $364 \mathrm{~g} / \mathrm{mol}$ is significantly larger than the $146 \mathrm{~g} / \mathrm{mol}$ of coumarin. It is not polar. It is a relatively long flexible molecule, unlike the sterically bulky known substrates. However, the inhibitor assays with starting 6:2 FTOH concentrations of $1000 \mathrm{ng} / \mathrm{mL}$ and $10000 \mathrm{ng} / \mathrm{mL}$ both showed that CYP 2A6 plays a role in the metabolism of 6:2 FTOH. Not only does the CYP 2A6 inhibition significantly reduce the metabolism of 6:2 FTOH, but it also had the greatest reduction in metabolic rate for both starting concentrations and surpassing the effects of CYP 3A4 inhibition. CYP 2A6 inhibition reduced the loss of FTOH by around 2.5 times that of the control assay for both concentrations tested. CYP 2A6 inhibition reduced the loss of FTOH by around 1.5 times that of the CYP 3A4 inhibition for both concentrations tested. The surprise of CYP 2A6 yielding a significant metabolic rate forced a new direction for the project. As this CYP has such a low percentage in the human liver and gut, this could significantly reduce the in-vivo toxicity of 6:2 FTOH. 6:2 
FTOH's fate in vivo is not solely dependent on the metabolism by CYPs. 6:2 FTOH has a hydroxyl group that can undergo phase II conjugation by Glutathione and Glucuronic acid. Elimination of 6:2 FTOH from the body will lead to the decrease of the toxic aldehyde metabolites. The low percentage of CYP 2 A6 could cause the in vivo toxicity of 6:2 FTOH to be heavily dependent on the rate of phase II conjugation.

While the results obtained with two 6:2 FTOH concentrations, with and without a CYP 2A6 selective inhibitor, provided preliminary information that this enzyme was responsible for 6:2 FTOH metabolism, we wanted to further explore the kinetics of CYP 2A6 on 6:2 FTOH metabolism. For the Michaelis-Menten curve, the low and high 6:2 FTOH concentration from the inhibitor assay fell within the linear zone. This was beneficial as the inhibitor concentration could be kept constant through all assays. The $K_{\mathrm{M}}$ values of the inhibited and uninhibited runs showed a significant difference. The $\mathrm{V}_{\max }$ obtained from the inhibited and uninhibited showed a significant difference. The Michaelis-Menten constants demonstrated that 6:2 FTOH metabolism by CYP 2A6 was being competitively inhibited by its selective inhibitor, tranylcypromine, $\mathrm{HCl}$.

Finally, the human recombinant CYP 2A6 assay was performed to confirm the effects of CYP 2A6 on 6:2 FTOH oxidation. Due to the similarities of CYP active sites, inhibitors could be effective for multiple CYPs in the liver microsomes. Tranylcypromine $\mathrm{HCl}$ is a potent inhibitor of CYP 2A6 ${ }^{[94]}$. However, it is also a moderate to low inhibitor of several other CYPs, primarily CYP 3A4 ${ }^{[94]}$. Therefore, the inhibition acquired from the use of tranylcypromine $\mathrm{HCl}$ could be from the inhibition of CYP 3A4 or another CYP. The human recombinant control and inhibition provided complete confirmation of CYP 2A6 metabolism of 6:2 FTOH. The human recombinant assay was also used to determine the rate of metabolism in the presence of a known amount of CYP 2A6. This would be useful when the human recombinant assay for the $3 \mathrm{~A} 4$ is performed. 
The comparison between both assay's metabolic rate will determine the CYP of greater importance in 6:2 FTOH metabolism.

CYP 3A4 is known for the metabolism of over half of prescribed drugs due to its abundance in the liver. CYP 3A4 metabolizes substrates of varying sizes. The known substrate of CYP 3A4 range from the relatively small acetaminophen and the bulky codeine as seen in figure 6.2. The CYP 3A subgroup constitutes a significant percentage (over 39\%) of the liver CYPs ${ }^{[22]}$. CYP 3A4 unlike 2E1 and 2A6 has a large and available active site. For this reason, many substrates are metabolized by CYP 3A4. In addition, the inhibitor ketoconazole is a large bulky compound suited for a specific inhibition for CYP 3A4.

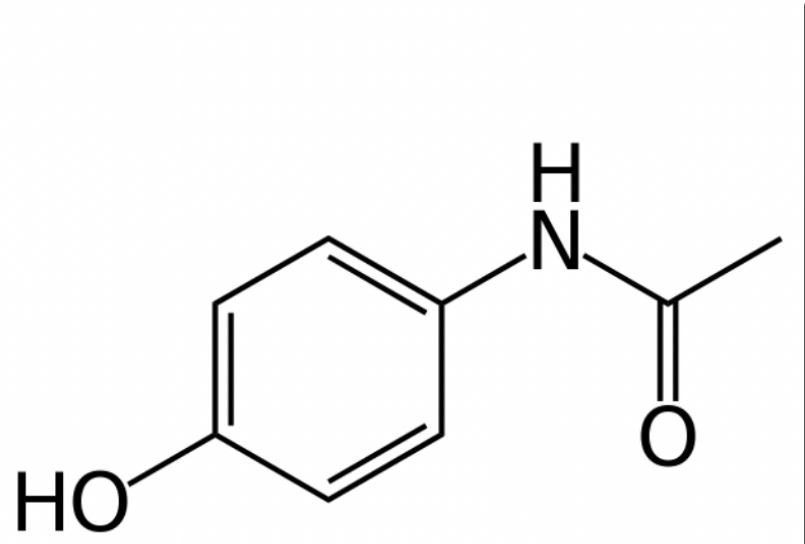

Acetaminophen

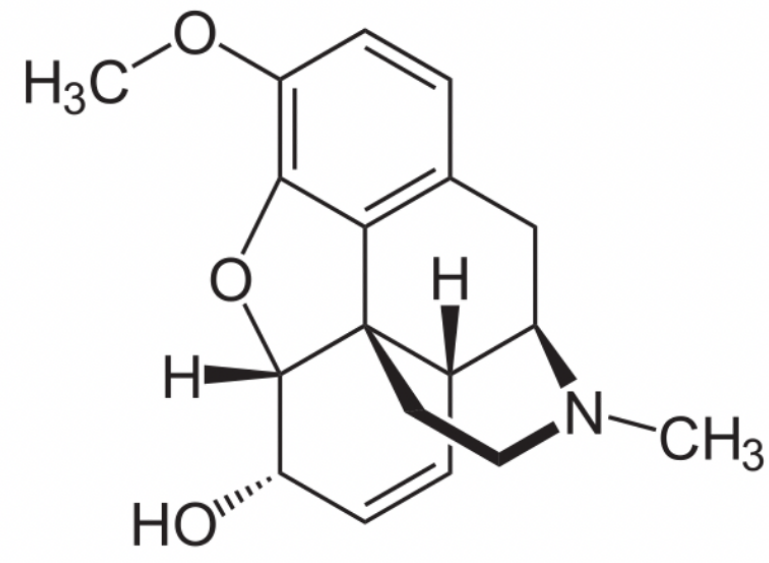

Codeine

Figure 6.2: CYP 3A4 substrates: Acetaminophen and Codeine

For 6:2 FTOH, the CYPs of interest are CYP 2A6 and CYP 3A4. If CYP 2A6 is the primary enzyme for the metabolism of 6:2 FTOH there is increased concern for hepatic toxicity 
resulting from its bioactive metabolites. If CYP $3 \mathrm{~A} 4$ is the primary enzyme for the metabolism of 6:2 FTOH, both hepatic and extra-hepatic (e.g., in the small intestine) may be observed. The route of 6:2 FTOH exposure could determine the organ most susceptible to its toxic metabolites with the account to first-pass metabolism. 


\section{Ongoing and Future Works}

The Identification of the CYPs responsible for 6:2 FTOH metabolism is just the first step in the determination of the inherent toxicity of 6 chained polyfluorinated compounds. CYP 2A6 and CYP 3A4 are vastly different CYPs concerning concentration in the liver, the primary organ of metabolism. The identification of CYP 2A6 points towards hepatoxicity. However, the comparison of $\mathrm{k}_{\mathrm{cat}}$ of both CYP 2A6 and CYP $3 \mathrm{~A} 4$ will need to be performed to determine which CYP 6:2 FTOH is primarily metabolized by. If it is shown to be CYP 3A4, extrahepatic toxicity is of greater concern.

Ongoing work involves the LC-MS/MS quantification of 6:2 FTCA and FTUCA production, two acid metabolites formed after 6:2 FTAL. The primary purpose of this project is to show that inactive microsomes, the creation of the acidic metabolites matches the trend of the FTOH loss. This supplements the idea that the FTOH metabolism is driving by CYP oxidation. In the presence of CYP2A6 and 3A4 inhibitors, inhibition of 6:2 FTOH metabolism will lead to inhibition of 6:2 FTCA and FTUCA production. 6:2 FTCA was not found at levels significantly higher than the instrument LOQ. This is because 6:2 FTCA rapidly dehydrogenates to form the 6:2 FTUCA. The 6:2 FTUCA production rate values are listed in supplementary table S7 and S8. The analysis of 6:2 FTUCA was performed for both the high $(10000 \mathrm{ng} / \mathrm{mL})$ and low $(1000$ $\mathrm{ng} / \mathrm{mL}$ ) concentration of 6:2 FTOH. Inhibiting CYP 3A4 and CYP 2A6 showed significant inhibition in the 6:2 FTUCA production, ANOVA results are presented in supplementary table S9. The inhibition of 6:2 FTUCA production correlates with the 6:2 FTOH metabolism results and increases the confidence of CYP 2A6 and CYP 3A4 being the enzymes responsible for 6:2 FTOH conversion. However, inhibition of all CYPs by $75 \mu \mathrm{M} 1$-aminobenzotriazole did not yield significant inhibition of 6:2 FTUCA production in $10000 \mathrm{ng} / \mathrm{mL}$ assay. 
Further analysis of the 6:2 FTUCA will be performed for the Michaelis-Menten assay samples to see if the 6:2 FTUCA production is competitively inhibited, just like the 6:2 FTOH loss. If the trend of competitive inhibition holds, HPLC-MS/MS analysis of the terminating polar metabolites will be performed on the human recombinant assay.

If the trend in competitive inhibition does not hold, other assays to determine additional enzymes involved in each oxidative step of 6:2 FTOH to its terminating PFCAs may be explored, as it is possible that the complex pathway is not entirely mediated by CYP enzymes. For example, conversion of the 6:2 FTAL to 6:2 FTCA may be controlled by aldehyde dehydrogenases or aldehyde oxidase. The 6:2 FTCA represents one of the major branching points in the biotransformation pathway of $6: 2 \mathrm{FTOH}$ as seen in figure 1.5. It contains an electronegative $\mathrm{OH}$ group that may be conjugated with a glucuronide via a UGT enzyme for phase II elimination, although currently no phase II products of 6:2 FTCA have been measured. Determining the enzyme(s) responsible for 6:2 FTCA formation and metabolism may be beneficial for further assessment of 6:2 FTOH toxicity in the human body, as well as its elimination.

Another ongoing project is to complete the inhibited CYP 3A4 Michaelis-Menten curve and test 6:2 FTOH metabolism in the presence of human recombinant CYP 3A4. This will follow the same method as the CYP 2A6 assays. The $\mathrm{CL}_{\text {int }}$ will be determined from the $\mathrm{V}_{\mathrm{Max}}$ and the $K_{\mathrm{M}}$. Comparison of both CLInt of CYP 2A6 and 3A4 will provide a greater understanding of the primary enzyme of metabolism in the human liver, as compared to the human recombinant enzyme assays.

Expanding the CYPs tested will be part of the future work done to complement the CYP 2A4 and CYP 3A4 assays. Enzymes localized in the liver were chosen for this project. Differing 
levels of CYPs in extrahepatic organs, such as the gastrointestinal tract or the lung, may have varied rates of 6:2 FTOH biotransformation. This brings up the question about which organ is more efficient in metabolizing 6:2 FTOH.

Expanding the microsomes tested to include the small intestine and lung, for example, should provide greater confidence in identifying not only the CYP responsible for 6:2 FTOH metabolism but also the most efficient organ or tissue. The primary routes of exposure for FTOHs are ingestion and inhalation ${ }^{[97]}$. The organs first in contact with 6:2 FTOH are therefore the GI tract and the lungs, respectively. Although 6:2 FTOH will eventually be transported to the liver, where the first-pass metabolism is primarily initiated, the presence of CYP in extrahepatic organs could lead to its activation before it reaches the liver. Measuring the relative clearance of 6:2 FTOH between organs could determine the severity of first-pass metabolism-based toxicity.

The discovery of the CYPs responsible for the metabolism of 6:2 FTOH is a significant step in determining the risk of 6:2 FTOH. Human contamination with FTOHs is widespread, from our drinking water to the animals and plants we consume. The true risk of FTOH contamination cannot be fully determined without the knowledge of its metabolic pathways leading to a toxic compound. The determination of the CYPs 2A6 and CYP 3A4 will help in understanding the efficiency of 6:2 FTOH conversion to the toxic 6:2 FTAL. CYP 2A6 and CYP 3A4 will help guide in vivo assays in the determination of localized toxicity of 6:2 FTOH. Due to the presence of polymorphic CYPs for both CYP 2 A6 and CYP 3A4, the severity of 6:2 FTOH metabolism could be skewed based on gender and ethnicity. This thesis project will be a stepping stone in our understanding of a new emerging FTOH of concern. The knowledge of how fast the toxic metabolites is metabolized from the starting FTOH, how often the starting 
FTOH are metabolised instead of eliminated and the preferred terminating metabolites all stem from the initial metabolism. 


\section{References}

1. Guengerich, F. P. (2001). Common and uncommon cytochrome P450 reactions related to metabolism and chemical toxicity. Chemical research in toxicology, 14(6), 611-650.

2. Meyer, U. A. (1996). Overview of enzymes of drug metabolism. Journal of pharmacokinetics and biopharmaceutics, 24(5), 449-459.

3. Lewis, D. F. (2003). Human cytochromes P450 associated with the phase 1 metabolism of drugs and other xenobiotics: a compilation of substrates and inhibitors of the CYP1, CYP2 and CYP3 families. Current medicinal chemistry, 10(19), 1955-1972.

4. Stella, V. J., Charman, W. N. A., \& Naringrekar, V. H. (1985). Prodrugs. Drugs, 29(5), 455-473.

5. Xu, C., Li, C. Y. T., \& Kong, A. N. T. (2005). Induction of phase I, II and III drug metabolism/transport by xenobiotics. Archives of Pharmacal research, 28(3), 249-268.

6. Jakoby, W. B., \& Ziegler, D. M. (1990). The enzymes of detoxication. The Journal of biological chemistry (Print), 265(34), 20715-20718.

7. Lamb, D. C., Lei, L., Warrilow, A. G., Lepesheva, G. I., Mullins, J. G., Waterman, M. R., \& Kelly, S. L. (2009). The first virally encoded cytochrome p450. Journal of virology, 83(16), 8266-8269. 
8. Nair, P. C., McKinnon, R. A., \& Miners, J. O. (2016). Cytochrome P450 structurefunction: insights from molecular dynamics simulations. Drug metabolism reviews, $48(3), 434-452$.

9. Meunier, B., De Visser, S. P., \& Shaik, S. (2004). Mechanism of oxidation reactions catalyzed by cytochrome P450 enzymes. Chemical reviews, 104(9), 3947-3980.

10. Belcher, J., McLean, K. J., Matthews, S., Woodward, L. S., Fisher, K., Rigby, S. E., ... \& Munro, A. W. (2014). Structure and biochemical properties of the alkene producing cytochrome P450 OleTJE (CYP152L1) from the Jeotgalicoccus sp. 8456 bacterium. Journal of Biological Chemistry, 289(10), 6535-6550.

11. Nabb, D. L., Szostek, B., Himmelstein, M. W., Mawn, M. P., Gargas, M. L., Sweeney, L. M., ... \& Fasano, W. J. (2007). In vitro metabolism of 8-2 fluorotelomer alcohol: interspecies comparisons and metabolic pathway refinement. Toxicological sciences, 100(2), 333-344.

12. Seelbach, K., Riebel, B., Hummel, W., Kula, M. R., Tishkov, V. I., Egorov, A. M., ... \& Kragl, U. (1996). A novel, efficient regenerating method of NADPH using a new formate dehydrogenase. Tetrahedron Letters, 37(9), 1377-1380.

13. Berka, K., Hendrychová, T., Anzenbacher, P., \& Otyepka, M. (2011). Membrane position of ibuprofen agrees with suggested access path entrance to cytochrome P450 2C9 active site. The journal of physical chemistry A, 115(41), 11248-11255. 
14. Zhang, H., Gao, N., Tian, X., Liu, T., Fang, Y., Zhou, J., ... \& Qiao, H. (2015). Content and activity of human liver microsomal protein and prediction of individual hepatic clearance in vivo. Scientific reports, 5(1), 1-12.

15. Yang, J., He, M. M., Niu, W., Wrighton, S. A., Li, L., Liu, Y., \& Li, C. (2012). Metabolic capabilities of cytochrome P450 enzymes in Chinese liver microsomes compared with those in Caucasian liver microsomes. British journal of clinical pharmacology, 73(2), 268-284.

16. Bezirtzoglou, E. E. V. (2012). Intestinal cytochromes P450 regulating the intestinal microbiota and its probiotic profile. Microbial ecology in health and disease, 23.

17. Nolin, T. D., Frye, R. F., \& Matzke, G. R. (2003). Hepatic drug metabolism and transport in patients with kidney disease. American journal of kidney diseases, 42(5), 906-925.

18. Carratt, S. A., Kovalchuk, N., Ding, X., \& Van Winkle, L. S. (2019). Metabolism and lung toxicity of inhaled naphthalene: Effects of postnatal age and sex. Toxicological Sciences, 170(2), 536-548.

19. Barnes, P. J. (2015). Club cells, their secretory protein, and COPD. Chest, 147(6), 14471448.

20. Gervot, L., Rochat, B., Gautier, J. C., Bohnenstengel, F., Kroemer, H., De Berardinis, V., ... \& De Waziers, I. (1999). Human CYP2B6: expression, inducibility and catalytic activities. Pharmacogenetics, 9(3), 295-306. 
21. Dai, Y., Iwanaga, K., Lin, Y. S., Hebert, M. F., Davis, C. L., Huang, W., ... \& Thummel, K. E. (2004). In vitro metabolism of cyclosporine A by human kidney CYP3A5. Biochemical pharmacology, 68(9), 1889-1902.

22. Gundert-Remy, U., Bernauer, U., Blömeke, B., Döring, B., Fabian, E., Goebel, C., ... \& Roos, P. H. (2014). Extrahepatic metabolism at the body's internal-external interfaces. Drug metabolism reviews, 46(3), 291-324.

23. Kirsch, P. (2013). Modern fluoroorganic chemistry: synthesis, reactivity, applications. John Wiley \& Sons.

24. Zaelke, Durwood; Borgford-Parnell, Nathan; Andersen., Stephen (11 January 2018). "Primer on HFCs" (PDF). Kristin Campbell, Xiaopu Sun, Dennis Clare,Claire Phillips, Stela Herschmann, Yuzhe PengLing, Alex Milgroom, Nancy J. Sherman. Institute for Governance \& Sustainable Development (IGSD): 5.

25. Yao, Y., Zhu, H., Li, B., Hu, H., Zhang, T., Yamazaki, E., ... \& Sun, H. (2014). Distribution and primary source analysis of per-and poly-fluoroalkyl substances with different chain lengths in surface and groundwater in two cities, North China. Ecotoxicology and environmental safety, 108, 318-328.

26. Dauchy, X., Boiteux, V., Bach, C., Rosin, C., \& Munoz, J. F. (2017). Per-and polyfluoroalkyl substances in firefighting foam concentrates and water samples collected near sites impacted by the use of these foams. Chemosphere, 183, 53-61.

27. Houde, M., Martin, J. W., Letcher, R. J., Solomon, K. R., \& Muir, D. C. (2006). Biological monitoring of polyfluoroalkyl substances: a review. Environmental science \& technology, 40(11), 3463-3473. 
28. Laitinen JA, Koponen J, Koikkalainen J, Kiviranta H (December 2014). "Firefighters' exposure to perfluoroalkyl acids and 2-butoxyethanol present in firefighting foams". Toxicology Letters. 231 (2): 227-32.

29. Domingo, J. L., \& Nadal, M. (2019). Human exposure to per-and polyfluoroalkyl substances (PFAS) through drinking water: a review of the recent scientific literature. Environmental research, 177, 108648.

30. Butt, C. M., Muir, D. C., \& Mabury, S. A. (2010). Biotransformation of the 8: 2 fluorotelomer acrylate in rainbow trout. 1. In vivo dietary exposure. Environmental toxicology and chemistry, 29(12), 2726-2735.

31. Liu, J., Wang, N., Szostek, B., Buck, R. C., Panciroli, P. K., Folsom, P. W., ... \& Bellin, C. A. (2010). 6-2 Fluorotelomer alcohol aerobic biodegradation in soil and mixed bacterial culture. Chemosphere, 78(4), 437-444.

32. Conder, J. M., Hoke, R. A., Wolf, W. D., Russell, M. H., \& Buck, R. C. (2008). Are PFCAs bioaccumulative? A critical review and comparison with regulatory criteria and persistent lipophilic compounds. Environmental science \& technology, 42(4), 995-1003.

33. Li, K., Gao, P., Xiang, P., Zhang, X., Cui, X., \& Ma, L. Q. (2017). Molecular mechanisms of PFOA-induced toxicity in animals and humans: Implications for health risks. Environment international, 99, 43-54.

34. Hagenaars, A., Vergauwen, L., Benoot, D., Laukens, K., \& Knapen, D. (2013). Mechanistic toxicity study of perfluorooctanoic acid in zebrafish suggests mitochondrial dysfunction to play a key role in PFOA toxicity. Chemosphere, 91(6), 844-856. 
35. Klaunig, J. E., Hocevar, B. A., \& Kamendulis, L. M. (2012). Mode of action analysis of perfluorooctanoic acid (PFOA) tumorigenicity and human relevance. Reproductive toxicology, 33(4), 410-418.

36. Lehmler, H. J. (2005). Synthesis of environmentally relevant fluorinated surfactants-a review. Chemosphere, 58(11), 1471-1496.

37. Fok, Sampson (2020). Biotransformation of 8:2 monosubstituted polyfluoroalkyl phosphate in rat \& human liver, intestine, and fecal in vitro suspensions published master's thesis]. Carleton University.

38. Schenker, U., Scheringer, M., Macleod, M., Martin, J. W., Cousins, I. T., \& Hungerbühler, K. (2008). Contribution of volatile precursor substances to the flux of perfluorooctanoate to the Arctic. Environmental science \& technology, 42(10), 37103716.

39. Jahnke, A., Ahrens, L., Ebinghaus, R., Berger, U., Barber, J. L., \& Temme, C. (2007). An improved method for the analysis of volatile polyfluorinated alkyl substances in environmental air samples. Analytical and bioanalytical chemistry, 387(3), 965-975.

40. Kim, M., Li, L. Y., Grace, J. R., \& Yue, C. (2015). Selecting reliable physicochemical properties of perfluoroalkyl and polyfluoroalkyl substances (PFASs) based on molecular descriptors. Environmental pollution, 196, 462-472.

41. Van Leeuwen, S. P. J., \& De Boer, J. (2007). Extraction and clean-up strategies for the analysis of poly-and perfluoroalkyl substances in environmental and human matrices. Journal of Chromatography A, 1153(1-2), 172-185.

42. Tian, Y., Yao, Y., Chang, S., Zhao, Z., Zhao, Y., Yuan, X., ... \& Sun, H. (2018). Occurrence and phase distribution of neutral and ionizable per-and polyfluoroalkyl 
substances (PFASs) in the atmosphere and plant leaves around landfills: A case study in Tianjin, China. Environmental science \& technology, 52(3), 1301-1310.

43. Lu, Z., Lu, R., Zheng, H., Yan, J., Song, L., Wang, J., .. \& Cai, M. (2018). Risk exposure assessment of per-and polyfluoroalkyl substances (PFASs) in drinking water and atmosphere in central eastern China. Environmental Science and Pollution Research, 25(10), 9311-9320.

44. Kim, S. K., Shoeib, M., Kim, K. S., \& Park, J. E. (2012). Indoor and outdoor poly-and perfluoroalkyl substances (PFASs) in Korea determined by passive air sampler. Environmental pollution, 162, 144-150.

45. Tian, Z., Kim, S. K., Shoeib, M., Oh, J. E., \& Park, J. E. (2016). Human exposure to perand polyfluoroalkyl substances (PFASs) via house dust in Korea: implication to exposure pathway. Science of the Total Environment, 553, 266-275.

46. Phillips, M. M., Dinglasan-Panlilio, M. J. A., Mabury, S. A., Solomon, K. R., \& Sibley, P. K. (2007). Fluorotelomer acids are more toxic than perfluorinated acids. Environmental science \& technology, 41(20), 7159-7163.

47. Stock, N. L., Furdui, V. I., Muir, D. C., \& Mabury, S. A. (2007). Perfluoroalkyl contaminants in the Canadian Arctic: evidence of atmospheric transport and local contamination. Environmental science \& technology, 41(10), 3529-3536.

48. Young, C. J., Furdui, V. I., Franklin, J., Koerner, R. M., Muir, D. C., \& Mabury, S. A. (2007). Perfluorinated acids in arctic snow: new evidence for atmospheric formation. Environmental science \& technology, 41(10), 3455-3461.

49. Stroski, K. (2019). Wastewater sources of priority contaminants in four Canadian Arctic Communities. [published master's thesis]. University of Manitoba. 
50. Hamid, H., \& Li, L. (2016). Role of wastewater treatment plant (WWTP) in environmental cycling of poly-and perfluoroalkyl (PFAS) compounds. Ecocycles, 2(2), $43-53$.

51. Gallen, C., Eaglesham, G., Drage, D., Nguyen, T. H., \& Mueller, J. F. (2018). A mass estimate of perfluoroalkyl substance (PFAS) release from Australian wastewater treatment plants. Chemosphere, 208, 975-983.

52. Szostek, B., \& Prickett, K. B. (2004). Determination of 8: 2 fluorotelomer alcohol in animal plasma and tissues by gas chromatography-mass spectrometry. Journal of Chromatography B, 813(1-2), 313-321.

53. Young, C. J., Furdui, V. I., Franklin, J., Koerner, R. M., Muir, D. C., \& Mabury, S. A. (2007). Perfluorinated acids in arctic snow: new evidence for atmospheric formation. Environmental science \& technology, 41(10), 3455-3461.

54. Ellis, D. A., Martin, J. W., De Silva, A. O., Mabury, S. A., Hurley, M. D., Sulbaek Andersen, M. P., \& Wallington, T. J. (2004). Degradation of fluorotelomer alcohols: a likely atmospheric source of perfluorinated carboxylic acids. Environmental science \& technology, 38(12), 3316-3321.

55. Li, Z. M., Guo, L. H., \& Ren, X. M. (2016). Biotransformation of 8: 2 fluorotelomer alcohol by recombinant human cytochrome P450s, human liver microsomes and human liver cytosol. Environmental Science: Processes \& Impacts, 18(5), 538-546.

56. Martin, J. W., Mabury, S. A., \& O’Brien, P. J. (2005). Metabolic products and pathways of fluorotelomer alcohols in isolated rat hepatocytes. Chemico-biological interactions, 155(3), 165-180. 
57. Rand, A. A., \& Mabury, S. A. (2013). Covalent binding of fluorotelomer unsaturated aldehydes (FTUALs) and carboxylic acids (FTUCAs) to proteins. Environmental science \& technology, 47(3), 1655-1663.

58. Rand, A. A., Rooney, J. P., Butt, C. M., Meyer, J. N., \& Mabury, S. A. (2014). Cellular toxicity associated with exposure to perfluorinated carboxylates (PFCAs) and their metabolic precursors. Chemical research in toxicology, 27(1), 42-50.

59. Eriksen, K. T., Raaschou-Nielsen, O., Sørensen, M., Roursgaard, M., Loft, S., \& Møller, P. (2010). Genotoxic potential of the perfluorinated chemicals PFOA, PFOS, PFBS, PFNA and PFHxA in human HepG2 cells. Mutation Research/Genetic Toxicology and Environmental Mutagenesis, 700(1-2), 39-43.

60. Henderson, W. M., \& Smith, M. A. (2007). Perfluorooctanoic acid and perfluorononanoic acid in fetal and neonatal mice following in utero exposure to 8-2 fluorotelomer alcohol. Toxicological sciences, 95(2), 452-461.

61. Ladics, G. S., Stadler, J. C., Makovec, G. T., Everds, N. E., \& Buck, R. C. (2005). Subchronic toxicity of a fluoroalkylethanol mixture in rats. Drug and chemical toxicology, 28(2), 135-158.

62. Liu, C., Yu, L., Deng, J., Lam, P. K., Wu, R. S., \& Zhou, B. (2009). Waterborne exposure to fluorotelomer alcohol 6: 2 FTOH alters plasma sex hormone and gene transcription in the hypothalamic-pituitary-gonadal (HPG) axis of zebrafish. Aquatic toxicology, 93(2-3), 131-137.

63. Liu, C., Zhang, X., Chang, H., Jones, P., Wiseman, S., Naile, J., ... \& Zhou, B. (2010). Effects of fluorotelomer alcohol 8: 2 FTOH on steroidogenesis in H295R cells: targeting the cAMP signalling cascade. Toxicology and applied pharmacology, 247(3), 222-228. 
64. Phillips, M. M., Dinglasan-Panlilio, M. J., Mabury, S. A., Solomon, K. R., \& Sibley, P. K. (2010). Chronic toxicity of fluorotelomer acids to Daphnia magna and Chironomus dilutus. Environmental toxicology and chemistry, 29(5), 1123-1131.

65. Stockholm Convention. Chemicals listed in Annex A. Stockholm Convention website. http://chm.pops.int/Implementation/Alternatives/AlternativestoPOPs/ChemicalslistedinA nnexA/tabid/5837/Default.aspx.

66. Stockholm Convention. Chemicals listed in Annex B. Stockholm Convention website. http://chm.pops.int/Implementation/Alternatives/AlternativestoPOPs/ChemicalslistedinA $\underline{\text { nnexB/tabid/5850/Default.aspx }}$

67. UN, United Nations, UN Treaties, Treaties. Retrieved from https://treaties.un.org/pages/ViewDetails.aspx?src=TREATY\&mtdsg_no=XXVII$15 \&$ chapter $=27$

68. Stockholm Convention. Overview. Retrieved from http://chm.pops.int/Implementation/EffectivenessEvaluation/Overview/tabid/369/Default. $\underline{\operatorname{aspx}}$

69. Stockholm Convention. The 12 initial POPs under the Stockholm Convention. Retrieved from http://chm.pops.int/TheConvention/ThePOPs/The12InitialPOPs/tabid/296/Default.aspx

70. Basic Information on PFAS. (2021, April 06). Retrieved from https://www.epa.gov/pfas/basic-information-pfas\#important

71. EPA Actions to Address PFAS. (2021, April 27). Retrieved from https://www.epa.gov/pfas/epa-actions-address-pfas 
72. Risk Assessment of Pollutants in Biosolids. (2021, February 16). Retrieved from https://www.epa.gov/biosolids/risk-assessment-pollutants-biosolids

73. Center for Food Safety and Applied Nutrition. (2020, July 31). FDA Announces the Voluntary Phase-Out by Industry of Certain PFAS Used. Retrieved May 27, 2021, from https://www.fda.gov/food/cfsan-constituent-updates/fda-announces-voluntary-phase-outindustry-certain-pfas-used-food-packaging

74. Health Canada. (2021, April 23). Government of Canada. Retrieved May 27, 2021, from https://www.canada.ca/en/health-canada/services/chemical-substances/other-chemicalsubstances-interest/long-chain-perfluorocarboxylic-acids-containing-9-20-carbon-atoms$\underline{\text { salts-precursors.html }}$

75. Health Canada. (2021, April 23). Government of Canada. Retrieved May 27, 2021, from https://www.canada.ca/en/health-canada/services/chemical-substances/other-chemicalsubstances-interest/per-polyfluoroalkyl-substances.html

76. Liu, X., Wang, H., Liang, X., \& Roberts, M. S. (2017). Hepatic metabolism in liver health and disease. In Liver Pathophysiology (pp. 391-400). Academic Press.

77. Nadal, M., \& L Domingo, J. (2014). Indoor dust levels of perfluoroalkyl substances (PFASs) and the role of ingestion as an exposure pathway: a review. Current Organic Chemistry, 18(17), 2200-2208.

78. Tian, Z., Kim, S. K., Shoeib, M., Oh, J. E., \& Park, J. E. (2016). Human exposure to perand polyfluoroalkyl substances (PFASs) via house dust in Korea: implication to exposure pathway. Science of the Total Environment, 553, 266-275. 
79. Lee, S., Kim, S., Park, J., Kim, H. J., Choi, G., Choi, S., ... \& Moon, H. B. (2018). Perfluoroalkyl substances (PFASs) in breast milk from Korea: Time-course trends, influencing factors, and infant exposure. Science of the Total Environment, 612, 286-292.

80. Schultes, L., Vestergren, R., Volkova, K., Westberg, E., Jacobson, T., \& Benskin, J. P. (2018). Per-and polyfluoroalkyl substances and fluorine mass balance in cosmetic products from the Swedish market: implications for environmental emissions and human exposure. Environmental Science: Processes \& Impacts, 20(12), 1680-1690.

81. Fujii, Y., Harada, K. H., \& Koizumi, A. (2013). Occurrence of perfluorinated carboxylic acids (PFCAs) in personal care products and compounding agents. Chemosphere, 93(3), $538-544$.

82. Pérez, F., Nadal, M., Navarro-Ortega, A., Fàbrega, F., Domingo, J. L., Barceló, D., \& Farré, M. (2013). Accumulation of perfluoroalkyl substances in human tissues. Environment international, 59, 354-362.

83. Krekels, E. H. J., Rower, J. E., Constance, J. E., Knibbe, C. A., \& Sherwin, C. M. (2017). Hepatic Drug Metabolism in Pediatric Patients. In Drug Metabolism in Diseases (pp. 181-206). Academic Press.

84. Faqi, A. S. (Ed.). (2012). A comprehensive guide to toxicology in preclinical drug development. Academic Press.

85. Greaves, A. (2016). Organophosphate Ester Contaminants in Herring Gulls (Larus argentatus) from the Great Lakes of North America: Bioaccumulation, Exposure, Pharmacokinetics and Trends (Doctoral dissertation, Carleton University). 
86. Enzymes structure and how it works. (2015, December 28). Retrieved April 23, 2021, from https://gotalktogetherdotcom.wordpress.com/2015/12/13/enzymes-structure-and-

\section{how-it-works/}

87. Lynch, T., \& Price, A. L. (2007). The effect of cytochrome P450 metabolism on drug response, interactions, and adverse effects. American family physician, 76(3), 391-396.

88. Hartman, J. H., Knott, K., \& Miller, G. P. (2014). CYP2E1 hydroxylation of aniline involves negative cooperativity. Biochemical pharmacology, 87(3), 523-533.

89. Rane, A., Wilkinson, G. R., \& Shand, D. G. (1977). Prediction of hepatic extraction ratio from in vitro measurement of intrinsic clearance. Journal of Pharmacology and Experimental Therapeutics, 200(2), 420-424.

90. Pritchard, J. F., Jurima-Romet, M., Reimer, M. L., Mortimer, E., Rolfe, B., \& Cayen, M. N. (2003). Making better drugs: decision gates in non-clinical drug development. Nature reviews Drug discovery, 2(7), 542-553.

91. Martin, J. W., Chan, K., Mabury, S. A., \& O’Brien, P. J. (2009). Bioactivation of fluorotelomer alcohols in isolated rat hepatocytes. Chemico-biological interactions, 177(3), 196-203.

92. Gonzalez, F. J. (2005). Role of cytochromes P450 in chemical toxicity and oxidative stress: studies with CYP2E1. Mutation Research/Fundamental and Molecular Mechanisms of Mutagenesis, 569(1-2), 101-110.

93. Rand, A. A., \& Mabury, S. A. (2012). In vitro interactions of biological nucleophiles with fluorotelomer unsaturated acids and aldehydes: fate and consequences. Environmental science \& technology, 46(13), 7398-7406. 
94. Taavitsainen, P., Juvonen, R., \& Pelkonen, O. (2001). In vitro inhibition of cytochrome P450 enzymes in human liver microsomes by a potent CYP2A6 inhibitor, trans-2phenylcyclopropylamine (tranylcypromine), and its nonamine analog, cyclopropylbenzene. Drug metabolism and disposition, 29(3), 217-222.

95. Su, T., Sheng, J. J., Lipinskas, T. W., \& Ding, X. (1996). Expression of CYP2A genes in rodent and human nasal mucosa. Drug Metabolism and Disposition, 24(8), 884-890.

96. Raunio, H., Rautio, A., Gullstén, H., \& Pelkonen, O. (2001). Polymorphisms of CYP2A6 and its practical consequences. British journal of clinical pharmacology, 52(4), 357-363.

97. Fujii, Y., Harada, K. H., \& Koizumi, A. (2013). Occurrence of perfluorinated carboxylic acids (PFCAs) in personal care products and compounding agents. Chemosphere, 93(3), 538-544. 


\section{Supplementary Data}

CONCENTRATION

(NG/ML)
RECOVERED CONCENTRATION(NG/ML)
PERCENT RECOVERY

$102 \pm 2.00 \%$

$2200 \pm 15$

$110 \pm 0.75 \%$

$3090 \pm 34$

$103 \pm 1.13 \%$

$3800 \pm 41$

$95 \pm 1.03 \%$

$6580 \pm 20$

$94 \pm 0.29 \%$

$7120 \pm 31$

$89 \pm 0.39 \%$

$6840 \pm 17$

$76 \pm 0.19 \%$

$6500 \pm 70$

$65 \pm 0.70 \%$

$9900 \pm 100$

$66 \pm 0.67 \%$

$12000 \pm 96$

$60 \pm 0.48 \%$

$27500 \pm 81$

$55 \pm 0.16 \%$

Table S1: Spike and recovery of 6:2 FTOH. Spike and recovery are obtained from 6:2 FTOH incubations for $2 \mathrm{hrs}$. 
STANDARD

RATE (NG/ML)

DEVIATION

UNINHIBITED
30 $\mu$ M CHLORAMPHENICOL
(CYP 2C19)
$68 \mu M$ ALLYL DISULFIDE (CYP
2E1)

16.41

0.39

30 $\mu$ M SULFAPHENAZOLE (CYP

2C9)

19.80

0.15

$75 \mu \mathrm{M} 1-$

AMINOBENZOTRIAZOLE (ALL

CYPS)

10.85

1.84

18$\mu$ M KETOCONAZOLE (CYP

3A4)

50 $\mu$ M TRANYLCYPROMINE,

HCL (CYP 2A6)

6.17

2.02

30 $\mu$ M QUINIDINE (CYP 2D6)

Table S2: Metabolic rates of 6:2 FTOH conversion in the presence of competitive inhibitors. The starting concentration of $6: 2 \mathrm{FTOH}$ is $1000 \mathrm{ng} / \mathrm{mL}$. Standard deviations are taken from $\mathrm{n}=3$ trials. 
STANDARD

RATE (NG/ML)

DEVIATION

\section{UNINHIBITED}

30 $\mu$ M CHLORAMPHENICOL

(CYP 2C19)

77.11

7.37

$68 \mu \mathrm{M}$ ALLYL DISULFIDE

(CYP 2E1)

88.17

4.69

$30 \mu M$ SULFAPHENAZOLE

(CYP 2C9)

$75 \mu \mathrm{M} 1-$

AMINOBENZOTRIAZOLE

(ALL CYPS)

18$\mu \mathrm{M}$ KETOCONAZOLE

(CYP 3A4)

50 $\mu$ M TRANYLCYPROMINE,

HCL (CYP 2A6)

30 $\mu$ M QUINIDINE (CYP 2D6)

Table S3: Metabolic rates of 6:2 FTOH conversion in the presence of competitive inhibitors.

The starting concentration of $6: 2 \mathrm{FTOH}$ is $10,000 \mathrm{ng} / \mathrm{mL}$. Standard deviations are taken from $\mathrm{n}=3$ trials. 


\section{6:2 FTOH RECOVERED (NG/ML)}

\begin{tabular}{|c|c|c|c|c|}
\hline & $17 \mathrm{Hrs}$ & $20 \mathrm{Hrs}$ & $25 \mathrm{Hrs}$ & $31 \mathrm{Hrs}$ \\
\hline NO INHIBITOR & $4811 \pm 11$ & $4709 \pm 20$ & $4520 \pm 10$ & $4464 \pm 15$ \\
\hline $\begin{array}{l}\text { TRANYLCYPROMINE, HCL } \\
(17.5 \mu M)\end{array}$ & $4523 \pm 5$ & $4510 \pm 25$ & $4502 \pm 13$ & $4497 \pm 13$ \\
\hline $\begin{array}{l}\text { TRANYLCYPROMINE, HCL } \\
(35.0 \mu M)\end{array}$ & $4493 \pm 17$ & $4492 \pm 13$ & $4493 \pm 15$ & $4488 \pm 20$ \\
\hline BLANK & $4497 \pm 20$ & $4500 \pm 19$ & $4499 \pm 20$ & $4493 \pm 17$ \\
\hline
\end{tabular}

Table S4: Metabolic rates of inhibited and uninhibited 6:2 FTOH conversion in the presence of Human recombinant CYP 2A6. The starting concentration of $6: 2 \mathrm{FTOH}$ is $10,000 \mathrm{ng} / \mathrm{mL}$. The starting concentration of CYP $2 \mathrm{~A} 6$ is $0.3 \mathrm{ng} / \mathrm{mL}$. Standard deviations are acquired from $\mathrm{n}=3$ trials. 
6:2 FTOH RECOVERED (NG/ML)

\begin{tabular}{l|rrrr}
\hline & 17 HOURS & 20 HOURS & 25 HOURS & 31 HOURS \\
\hline NO INHIBITOR & $948 \pm 3$ & $919 \pm 11$ & $875 \pm 5$ & $822 \pm 3$ \\
& & & & \\
TRANYLCYPROMINE, HCL & $1090 \pm 3$ & $1090 \pm 6$ & $1085 \pm 5$ & $1083 \pm 3$ \\
$(\mathbf{3 5 . 0} \boldsymbol{\mu M})$ & $1111 \pm 15$ & $1109 \pm 20$ & $1110 \pm 10$ & $1107 \pm 13$ \\
BLANK & & & & \\
& & & &
\end{tabular}

Table S5: Metabolic rates of inhibited and uninhibited 6:2 FTOH conversion in the presence of Human recombinant CYP 2A6. The starting concentration of 6:2 FTOH is $1000 \mathrm{ng} / \mathrm{mL}$. The starting concentration of CYP 2A6 is $0.3 \mathrm{ng} / \mathrm{mL}$. Standard deviations are acquired from $\mathrm{n}=3$ trials. 


\section{CONCENTRATION 6:2 FTOH LOSS \\ STD DEV SPECIFIC RATE \\ (ng/mL) 6:2 FTOH LOSS （ng/mL min) ～(ng/min mg) \\ RATE}

(ng/mL min)

\begin{tabular}{|c|c|c|c|c|}
\hline INHIBITED & 1000.00 & 6.69 & 1.50 & 33.47 \\
\hline (CYP 2A6) & 2000.00 & 10.48 & 1.00 & 52.42 \\
\hline \multirow[t]{7}{*}{ MICROSOMES } & 4000.00 & 21.63 & 1.10 & 108.13 \\
\hline & 7000.00 & 21.10 & 0.79 & 105.50 \\
\hline & 8000.00 & 30.15 & 1.49 & 150.77 \\
\hline & 9000.00 & 35.78 & 3.00 & 178.91 \\
\hline & 15000.00 & 46.54 & 2.50 & 232.69 \\
\hline & 20000.00 & 51.86 & 1.91 & 259.28 \\
\hline & 50000.00 & 56.09 & 2.97 & 280.45 \\
\hline UNINHIBITED & 1000.00 & 12.11 & 1.30 & 60.54 \\
\hline \multirow[t]{8}{*}{ MICROSOMES } & 2000.00 & 23.98 & 0.20 & 119.89 \\
\hline & 4000.00 & 37.77 & 1.65 & 188.85 \\
\hline & 7000.00 & 35.92 & 1.50 & 179.62 \\
\hline & 8000.00 & 43.71 & 1.49 & 218.57 \\
\hline & 9000.00 & 43.79 & 3.27 & 218.94 \\
\hline & 15000.00 & 52.31 & 3.88 & 261.55 \\
\hline & 20000.00 & 60.48 & 1.91 & 302.38 \\
\hline & 50000.00 & 64.00 & 2.32 & 320.00 \\
\hline
\end{tabular}


Table S6: Rate and specific rate of 6:2 FTOH in uninhibited and inhibited (CYP 2A6) microsomes. The inhibition is from the addition of $50.0 \mu \mathrm{M}$ of Tranylcypromine, HCL. Standard deviations are acquired from $n=3$ trials.

\section{6:2 FTUCA \\ STANDARD DEVIATION}

\section{PRODUCTION}

RATE (NG/ML)

\begin{tabular}{l|cc}
\hline UNINHIBITED & 0.118 & 0.012 \\
CYP 2C19 $(30 \mu \mathrm{M})$ & 0.052 & 0.005 \\
CYP 2E1 $(68 \mu \mathrm{M})$ & 0.112 & 0.007 \\
CYP 2C9 $(30 \mu \mathrm{M})$ & 0.094 & 0.017 \\
CYP 3A4 $(18 \mu \mathrm{M})$ & 0.051 & 0.003 \\
CYP 2A6 $(50 \mu \mathrm{M})$ & 0.024 & 0.010 \\
CYP 2D6 $(30 \mu \mathrm{M})$ & & 0.013 \\
& & \\
ALL CYPS $(75 \mu \mathrm{M})$ & 0.222 & 0.017
\end{tabular}

Table S7: Metabolic rates of 6:2 FTUCA conversion in the presence of competitive inhibitors. The starting concentration of $6: 2 \mathrm{FTOH}$ is $1000 \mathrm{ng} / \mathrm{mL}$. Standard deviations are taken from $\mathrm{n}=3$ trials. 
$(\mathrm{NG} / \mathrm{ML})$

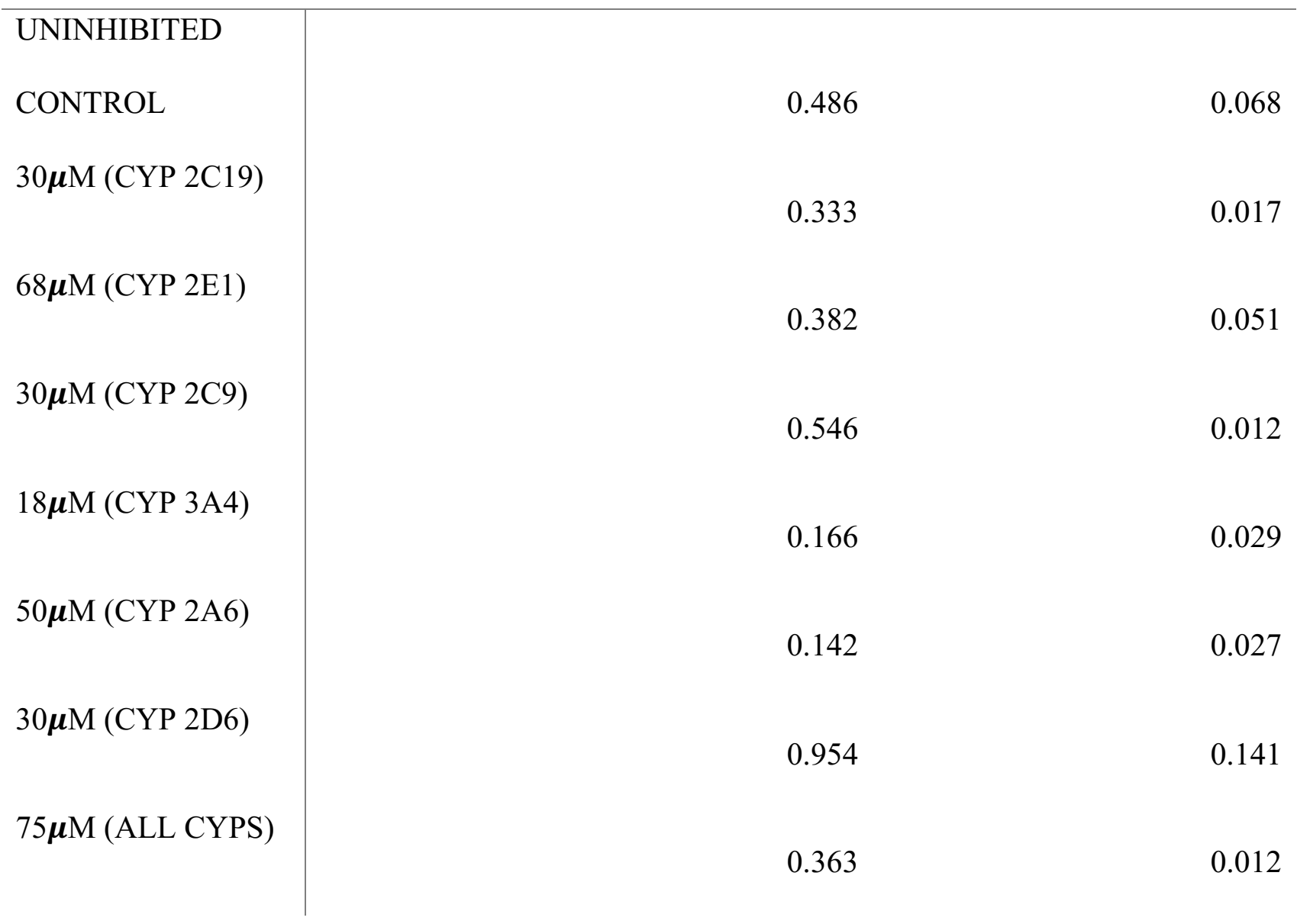

Table S8: Metabolic rates of 6:2 FTUCA conversion in the presence of competitive inhibitors.

The starting concentration of $6: 2 \mathrm{FTOH}$ is $10,000 \mathrm{ng} / \mathrm{mL}$. Standard deviations are taken from $\mathrm{n}=3$ trials. 
ANOVA p-values

(10,000ng/mL)
ANOVA p-values

$(1,000 \mathrm{ng} / \mathrm{mL})$
CYP 2C19 (30uM)

CYP 2E1 (68uM)

CYP 2C9 (30uM)

ALL CYPS (75uM)

CYP 3A4 (18uM)

CYP 2A6 (50uM)

CYP 2D6 (30uM)

All trials

$0.08 \quad 0.0030$

0.15

0.9500

0.11

0.5400

0.17

0.0070

0.002

0.0007

0.002

0.0002

0.001

0.0020

4.33E-10

2.6680E-12

Table S9: The p-values corresponding with a one-way ANOVA test between the uninhibited and inhibited 6:2 FTUCA production rates at 1,000 and 10,000 ng/mL. "All trials" signify the ANOVA test comparing the uninhibited with all the inhibited trials. 
